\title{
From Molecules to Management: Mechanisms and Consequences of Locust Phase Polyphenism
}

Darron A. Cullen ${ }^{a *}$, Arianne Cease ${ }^{b, c}$, Alexandre V. Latchininsky ${ }^{d}$, Amir Ayali e ${ }^{\text {, Kevin Berry }}{ }^{\mathrm{f}}$, Jerome Buhl $^{\mathrm{g}}$, Rien De Keyser ${ }^{\mathrm{h}}$, Bert Foquet ${ }^{\mathrm{j}}$, Joleen C. Hadrich ${ }^{\mathrm{k}}$, Tom Matheson ${ }^{\mathrm{h}}$, Swidbert R. $\mathrm{Ott}^{\mathrm{h}}$, Mario A. Poot-Pech ${ }^{\mathrm{m}}$, Brian E. Robinson ${ }^{\mathrm{n}}$, Jonathan M. Smith ${ }^{\mathrm{h}}$, Hojun Song ${ }^{\mathrm{j}}$, Gregory A. Sword $^{\mathrm{j}}$, Jozef Vanden Broeck ${ }^{\mathrm{a}}$, Rik Verdonck ${ }^{\mathrm{a}}$, Heleen Verlinden ${ }^{\mathrm{a}}$, Stephen M. Rogers ${ }^{\mathrm{b}, \mathrm{o}}$

*Corresponding author: darron.cullen@bio.kuleuven.be

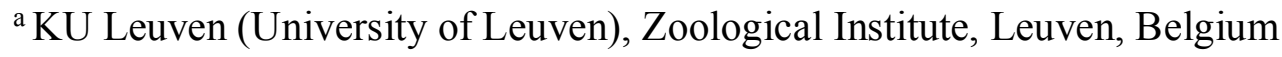

${ }^{\mathrm{b}}$ Arizona State University, School of Life Sciences, Tempe, Arizona, U.S.A.

${ }^{c}$ Arizona State University, School of Sustainability, Tempe, Arizona, U.S.A.

${ }^{\mathrm{d}}$ University of Wyoming, Ecosystem Science and Management, Laramie, Wyoming, U.S.A.

e Tel-Aviv University, Department of Zoology, Tel-Aviv, Israel

${ }^{\mathrm{f}}$ University of Alaska Anchorage, Institute of Social and Economic Research, Anchorage, Alaska, U.S.A.

g University of Adelaide, School of Agriculture, Food and Wine, Adelaide, Australia

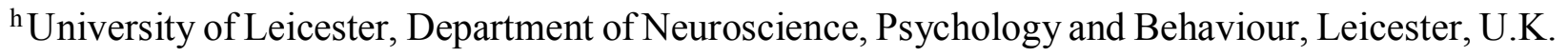

j Texas A\&M University, Department of Entomology, College Station, Texas, U.S.A.

${ }^{\mathrm{k}}$ Colorado State University, Department of Agricultural \& Resource Economics, Fort Collins, Colorado, U.S.A.

m Yucatán State Plant Protection Committee (Locust Program), Mérida, Yucatán, México

${ }^{\mathrm{n}}$ McGill University, Department of Geography, Montreal, Quebec, Canada

${ }^{\circ}$ University of Cambridge, Department of Zoology, Cambridge, U.K. 


\section{Abstract}

Locusts are grasshoppers (Orthoptera: Acrididae) that are characterised by their capacity for extreme population density-dependent polyphenism, transforming between a cryptic solitarious phase that avoids other locusts, and a swarming gregarious phase that aggregates and undergoes collective migration. The two phases differ in many aspects of behaviour, physiology and ecology, making locusts a useful model through which to investigate the phenotypic interface of molecular processes and environmental cues. This review summarises recent progress in understanding the mechanisms and consequences of locust phase change, from differential gene expression and epigenetic regulation through to neuronal plasticity and altered behaviour. The impact of techniques such as RNA interference (RNAi), and the sequencing of the first locust genome is discussed, and we consider the evidence from comparative analyses between related locust species for the possible evolution of locust-like phenotypic plasticity. Collective movement, and new ways of measuring the behaviour of both migrating bands in the field and individuals in the laboratory, are analysed. We also examine the environmental factors that affect phase change, along with the wider impact of land use and management strategies that may unwittingly create environments conducive to outbreaks. Finally, we consider the human costs of locust swarming behaviour, and use combined social, economic and environmental approaches to suggest potential ways forward for locust monitoring and management.

Key Words: Locust phase polyphenism, phenotypic plasticity, epigenetics, behaviour, biogenic amine, collective movement, environmental stimuli, human-locust interactions, Schistocerca, Locusta 


\section{Contents}

$1 \quad$ Introduction 4

1.1 Scope and intent of the present review 4

1.2 Nomenclature, and a note on laboratory strains 6

1.3 Recent locust outbreaks 8

2 Evolution and diversity of swarming grasshoppers 11

3 Measuring phase-related behaviour 17

3.1 Recent behavioural studies 18

3.2 Suggestions for future studies 26

4 Proximal stimuli inducing phase change 27

$5 \quad$ Biogenic amine signalling $\quad 30$

5.1 Phylogeny and pharmacology of biogenic amine receptors 31

5.2 Involvement of biogenic amines and their receptors in locust phase transition 37

$6 \quad$ Genomics, transcriptomics and epigenetics 43

6.1 Availability of sequence information 43

6.2 Transcriptomic differences between long-term phases 44

6.3 The transcriptome during phase transition $\quad 46$

6.4 Mobile elements 46

6.5 DNA methylation in locusts 47

$7 \quad$ Neurophysiological consequences of phase change $\quad 52$

7.1 Social complexity and brain size 53

7.2 Learning abilities of solitarious and gregarious locusts 54

7.3 Phase-related differences in daily rhythms of activity 58

7.4 Neurophysiological correlates of diel patterns of activity 61

7.5 Circadian clock genes in Schistocerca gregaria 62

$8 \quad$ Collective behaviour 63

8.1 Laboratory studies of locust collective behaviour 63

8.2 The need for large scale movement data 68

9 Environmental stimuli affecting phase change 71

10 Ecology and landscape-level processes 76

10.1 Remote sensing 76

10.2 Climate change 80

10.3 Locust ecology case study: The Central American Locust, Schistocerca piceifrons 83

11 Human - locust interactions 86

12 Concluding remarks 90

Acknowledgements 93

References 94 


\section{Introduction}

\subsection{SCOPE AND INTENT OF THE PRESENT REVIEW}

The present review arose out of two symposia held at the XXV International Congress of Entomology, Orlando, Florida (September 2016), which succeeded in bringing together researchers representing many of the groups around the world currently working on all aspects of locust phase polyphenism and its wider consequences. Most of the attendees at these symposia agreed to write synopses of recent progress in different areas of locust research, each according to their interests and expertise, which form the core of this paper. We have three objectives in this review. First, to act as an update to the seminal review of Pener and Simpson (2009) and so the emphasis here is on papers published in the eight years since that chapter. We refer the reader to other reviews, such as Buhl and Rogers (2016), Ernst et al. (2015), Ariel and Ayali (2015), Wang and Kang (2013), Burrows et al. (2011), Verlinden et al. (2009), Pener and Yerulshami (1998), Pener $(1991)$ and the books by Uvarov $(1966,1977)$ for material covering different aspects of the entire history of locust phase change research.

Our second objective is to cover as broad a spectrum of current research interests in locust phase change as possible. As expected, we review the dramatic developments in our understanding of the molecular, epigenetic and physiological mechanisms that drive the phase change process, which have been made possible by the rapid progress of molecular and behavioural research techniques (Cullen, De Keyser, Ott, Rogers, Smith, Vanden Broeck, Verdonck and Verlinden). Perhaps the most prominent advance over recent years has been the publication of a draft genome sequence for the Migratory Locust, Locusta migratoria (Wang et al., 2014), bringing locust phase research into the genomic era for the first time. This genome, and other locust sequence data, has greatly facilitated gene-driven studies of locust physiology, many of which have used RNA interference (RNAi), which has become a powerful tool in locust research. RNAi is a method of post-transcriptional gene silencing, which employs double-stranded RNA (dsRNA) complementary to an RNA transcript of interest (typically protein-coding mRNA) to generate functional knockdowns via a highly-conserved enzymatic pathway, and has been used to great effect across a wide range of insects (Bellés, 2010; Cullen, 2012). RNAi causes a potent and specific systemic gene knockdown in locusts, which can be achieved by a simple injection of 
dsRNA into the body cavity (Wynant et al., 2012). RNAi has been used to corroborate the dramatic role of the neuropeptide [His ${ }^{7}$ ] corazonin in causing the black colouration and some morphometric characteristics of gregarious locusts (Sugahara et al., 2015), as well being an important tool in investigating the molecular signalling pathways underlying behavioural phase change (see Sections 4 and 5). Intriguingly, crowding solitarious locusts leads to a rapid but transient increase (30 min to $1 \mathrm{~h}$ ), in the expression of genes involved in mediating the RNAi response (Wynant et al., 2015). Other advances include automated video tracking, and new ways of interpreting the resulting data, which have improved our methods for assaying locust behavioural phase (e.g. Cullen et al., 2010, 2012; Stettin and Ott, 2012; Ma et al., 2011) and underpin many of the physiological experiments discussed throughout this review.

We go on to survey how the altered lifestyles and ecology of solitarious and gregarious locusts necessitate altered brain structures, neural processing and learning capacities to deal with the different requirements imposed by altered social and physical environments (Matheson and Rogers). The self-organisation of gregarious locusts into coherent migratory bands, potentially comprised of millions of individuals, all with a mostly uniform direction of marching is one of their most striking behaviours. Recent progress in the modelling and measuring of collective motion in locusts using new methodologies is presented (Ayali, Buhl and Sword).

We also cover work using comparative analyses of locusts and other acridid species showing only limited polyphenism, to give new insights into the evolution of full densitydependent phase change (Foquet and Song). The ecology of locusts, and the ways in which landscape, vegetation type and land use may influence the likelihood of locust outbreaks and plagues are also considered in some more detail than in Pener and Simpson (2009) (Cease and Latchininsky) with the Central American Locust, Schistocerca piceifrons being used as a specific case study (Poot-Pech and Song). Finally, we consider the costs and consequences of locust swarms and the damage they inflict from a socioeconomic perspective (Berry, Hadrich and Robinson). Much research on locusts, from the molecular to the ecological is justified on the ground of the economic damage they inflict, yet this is often only loosely tied to quantitative analyses of the actual economic, and often surprising wider social, costs that they inflict. Here we include a brief survey of the priorities, methods, models and major findings of some of the social scientists working on human-locust interactions in the context of wider land-use strategies. As a 
result, this paper aims to bridge the mechanisms and consequences of locust phase polyphenism from molecules to livelihoods.

The third and final objective is that this review will stand as a means to introduce the Global Locust Initiative (locust.asu.edu). This initiative's purpose is to enable innovative advances in locust research and management by promoting interdisciplinary systems- and solutions-oriented approaches. The initiative is based on three pillars: advancing fundamental research, creating and maintaining a global network, and developing local solutions to the global challenge of locust plagues. The initiative grew out of many conversations among researchers, government and nongovernment agencies, and farmers' groups around the world. We will formally launch the Global Locust Initiative in 2018 and aim to keep it broadly inclusive and transparent.

\subsection{NOMENCLATURE, AND A NOTE ON LABORATORY STRAINS}

This review follows the standard convention of naming species by both their common and scientific names in the first instance, and abbreviated scientific name thereafter. Common names are sometimes reused to aid readability, and the reader is also referred to Table 1 for a full list of all accepted locust species, along with naming authorities and geographic range. Table 1 also gives the same details for all other species of Orthoptera named in the review.

Much of the work discussed in this review focusses on two species of locust reared in laboratory colonies: The Desert Locust, Schistocerca gregaria and the Migratory Locust, Locusta migratoria. Different laboratory strains of the same species or subspecies can vary in many aspects of their physiology and behaviour, reflecting their region of origin, rearing conditions, and the number of generations in captivity. For further discussion see Berthier et al. (2010), who performed population genetic and inbreeding analyses across a range of laboratory locust strains.

Discussions of laboratory-reared S. gregaria will almost invariably refer to colonies derived from wild populations of the nominate phase-changing northern race, S. gregaria gregaria (Forskål, 1775), which has been brought into captivity from many regions of North Africa and the Middle East. By contrast, the southern race, S. gregaria flaviventris (Burmeister, 1838) does not undergo phase change (Chapuis et al., 2017), and is therefore highly unlikely to have been the founder subspecies for any laboratory colony used to successfully study this form of behavioural plasticity. 
As the most widely distributed grasshopper species in the world, the situation for $L$. migratoria is more complex. Many subspecies of L. migratoria have been historically recognised, based primarily on their geographic range; this is reflected in the literature for both laboratory-and field-based studies. Ma et al. (2012) removed much of the uncertainty about Locusta subspecies in a comprehensive phylogeographical analysis, in which they sequenced three mitochondrial genes for 263 individuals across 53 worldwide sampling sites, along with 65 complete mitochondrial genomes. The authors tentatively collapsed all subspecies into just two, based on broadly northern and southern lineages: the nominate Asian Migratory Locust, L. migratoria migratoria (Linnaeus, 1758), and the African Migratory Locust, L. migratoria migratorioides (Reiche and Fairmaire, 1849). The latter race is of particular interest; it subsumed, perhaps unsurprisingly, the Malagasy Migratory Locust, L. migratoria capito Saussure, 1884 from Madagascar, but also the Oriental (L. migratoria manilensis Meyen, 1835) and Tibetan (L. migratoria tibetensis Chen, 1963) subspecies (Ma et al., 2012). Throughout the current review, we will use the recent reclassification of Ma et al. (2012) where a subspecies is given, but will also mention the defunct synonym given by the authors where applicable.

A further result of this reclassification is that some countries around central and east Asia are home to both of the currently accepted subspecies of L. migratoria. This is particularly obvious in China, which has wild populations of L. migratoria migratoria in the north and L. migratoria migratorioides in the south. Much of the molecular and physiological work discussed throughout this review was performed at the Chinese Academy of Sciences, Beijing. Their laboratory colony originates from the neighbouring northern provinces of Hebei and Shandong and, since they therefore belong to the nominate subspecies L. migratoria migratoria, the authors generally refer to their laboratory strain as simply L. migratoria L. (Professor Kang Le, pers. comm. to D. Cullen; Ma et al., 2012). In their highly-anticipated genome description (Wang et al., 2014), the authors also used DNA from a male locust captured in the southern island province of Hainan, which would be L. migratoria migratorioides according to Ma et al. (2012). For all studies, and for all species or subspecies, the reader is advised to contact the relevant authors where taxonomic information and/or founder colony location is unclear.

\subsection{RECENT LOCUST OUTBREAKS}


In the past decade there have been widespread reports of locust upsurges around the world. The infamous Desert Locust, Schistocerca gregaria can invade up to 29 million $\mathrm{km}^{2}$ from Northern Africa to India, nearly 20\% of the Earth's land area (Popov et al., 1991). The last major invasion of $S$. gregaria was in 2003-2005 when 13 million ha were treated with pesticides in 22 countries (Belayneh, 2005). The Food and Agriculture Organization of the United Nations (FAO) estimated this plague caused crop losses of $80-100 \%$ in afflicted regions, mostly in Sub-Saharan Africa (Brader et al., 2006) and cost over \$500 million USD to control (Belayneh, 2005). While there has not been an $S$. gregaria plague in the past 10 years, there have been numerous localised outbreaks that have led to swarm formation (FAO Locust Watch: http://www.fao.org/ag/locusts). In 2008, there were swarms in Ethiopia and Saudi Arabia. Widespread outbreaks occurred between 2012 and 2013 from Mauritania in the western region of this species' range across to Israel, Yemen and Saudi Arabia in the near east range. In Israel alone, control efforts by the Israeli Ministry of Agriculture were estimated to cost $\$ 4$ million USD, with more than $80 \%$ of this spent on nymphal band control (Israeli Ministry of Agriculture and Nature and Parks Authority, pers. comm. to A. Ayali). Fortunately, damage to agriculture was very limited as the locusts were mostly confined to the Negev desert. The locust situation remained serious along the Red Sea coast and Horn of Africa through to March 2015. From 2015 to May 2017, the threat fluctuated in Yemen, Saudi Arabia, Sudan, and Mauritania. As of May 2017, the risk and threat to crops have declined, but the situation is unclear in Yemen where surveys cannot be conducted.

In east, central, and southern Africa, several locusts are a periodic threat to food security including the Brown Locust, Locustana pardalina, the African Migratory Locust, Locusta migratoria migratorioides, and the Red Locust, Nomadacris septemfasciata. Vast L. migratoria migratorioides plagues covering parts of western, central, and southern Africa were common historically in the late 1800 s and mid $1900 \mathrm{~s}$, but $N$. septemfasciata has likely posed the biggest threat in the past decade. In 2008-2009, Malawi, Mozambique, Tanzania and Zambia were hit with upsurges of $N$. septemfasciata. The FAO coordinated a campaign together with the International Red Locust Control Organization for Central and Southern Africa (IRLCO-CSA). This campaign was the first time biopesticides (the mycopesticide based on Metarhizium anisopliae) were used on a large scale against locusts in Africa. (FAO bulletin: http://www.fao.org/agriculture/crops/news-eventsbulletins/detail/en/item/40241/icode/3/?no_cache=1). Without intervention, the FAO estimated 
the invasion could have affected the food crops of around 15 million people (http://www.fao.org/news/story/en/item/21084/icode/). In January 2017, Zambia experienced outbreaks of both L. migratoria migratorioides and N. septemfasciata, which damaged over 10,000 ha of grassland and cropland. In February 2017, the director of the IRLCO-CSA said \$4 million USD would be needed to tackle the problem (http://www.herald.co.zw/region-needs-4m-to-fightlocusts/). As of May 2017, the outbreaks are seen as a current threat to food security across central and southern Africa.

In Madagascar, a plague of Malagasy Migratory Locusts, L. migratoria migratorioides (previously known as L. migratoria capito) began in June 2012 and threatened the livelihoods and food security of 13 million people (60\% of the country's population). During three locust campaigns in 2013-2016, the locusts were controlled on 2.3 million ha at a cost of $\$ 37$ million USD (see http://www.fao.org/emergencies/crisis/madagascar-locust/en/).

Kazakhstan, Russia and other countries of the Caucasus and Central Asia experienced high levels of infestations by the Asian Migratory Locust, L. migratoria migratoria, Italian Locust, Calliptamus italicus, and Moroccan Locust, Dociostaurus maroccanus in the past decade. From 2010 to 2016, infested areas in this region varied from 7.1 to 11.2 million ha per year. Anti-locust treatments peaked in 2014 with 6.7 million ha out of which 4.7 million ha were treated in Kazakhstan, principally against $C a$. italicus (see http:/www.fao.org/ag/locustsCCA/en/index.html).

Records of L. migratoria outbreaks in China reach back more than 1,000 years (Stige et al., 2007) and they remain a threat to agriculture in present times. For example, from 2012 to 2016, L. migratoria outbreaks covered about 1.5 million ha (Long Zhang, unpublished data, pers. comm. to A. Cease). Both subspecies of L. migratoria are common in China, with several synonyms common in the literature (Ma et al., 2012; see Section 1.2). China has made great strides to increase biopesticide use in place of synthetic pesticides, specifically the fungus Metarhizium acridum (Bischoff et al., 2009) and the microsporidian Paranosema locustae. In 2004, around 5\% of locust treatments used these biopesticides, but in 2017 they account for more than 30\%. At over 100,000 ha treated, this is the largest application of biopesticides against locusts (Long Zhang and David Hunter, unpublished data, pers. comm. to A. Cease).

In eastern Asia, the Yellow-Spined Bamboo Locust, Ceracris kiangsu is a periodic threat. In 2015, Laos was affected by an unprecedented outbreak Ce. kiangsu, which led to food insecurity 
and loss of livelihood for more than 20,000 people by July 2015 (http://www.fao.org/laos/news/detail-events/en/c/414367/). Despite management efforts by the FAO and the Laos and Chinese governments, as of May 2017 Ce. kiangsu is still at plague proportions (https:/www.laotiantimes.com/2017/05/05/locusts-swarm-northern-laos-wreakinghavoc-crops/).

In 2010, Australia experienced one of the worst plagues of the Australian Plague Locust, Chortoicetes terminifera in 40 years (Deveson, 2011). This plague was established following successful breeding in New South Wales and Queensland, and exacerbated by an intense La Niña in March 2010 that increased populations further (Deveson, 2013). At its peak, over 2,000 landholders reported high-density bands; the plague subsided in early 2011 after control by the Australian Plague Locust Commission and state-level agencies (Deveson and Woodman, 2014). Since 2011, outbreaks have been reported across the country annually, with intense localised damage in some areas but no widespread plagues (Australian Government: http://www.agriculture.gov.au/pests-diseases-weeds/locusts).

The Americas are home to a few locust species that have had outbreaks recently including the Central American Locust, Schistocerca piceifrons (Poot-Pech, 2017; see Section 10.3), the South American Locust, Schistocerca cancellata, and the Moruga grasshopper, Coscineuta virens. Co. virens exhibits some characteristics of locust phase polyphenism and has periodic outbreaks in its restricted range in Trinidad (Song, 2011). There were outbreaks in 2010, then again starting in 2013 and continuing as of May 2017 (http://www.trinidadexpress.com/20170517/news/1l).

The South American Locust, Schistocerca cancellata was the most destructive agricultural pest in Argentina during the late 1800s to early 1900s. However, after an extensive 1954 control campaign involving 12,000 tons of pesticide (de Wysiecki and Lange, 2005) its numbers decreased considerably with smaller outbreaks in 1961, 1989, and 2010 (Hunter and Cosenzo, 1990; Barrientos Lozano, 2011). In the latter half of 2015, an upsurge level unprecedented in 60 years originated from the long-standing recession zone in NW Argentina and spread through a large region including Santiago del Estero, Catamarca, Tucuman, Salta, Chaco, and Cordoba. The 2015 swarm caused fear in many rural areas because this locust was historically devastating; however, the swarms were controlled and there was little agricultural impact from this upsurge (Wilda Ramírez and Hector Medina, SENASA; Eduardo Trumper, INTA, Argentina, pers. comm.). In January 2017 in Bolivia, a more than 50-year lack of reported locust activity was interrupted by 
the appearance of a swarm near Santa Cruz. In February 2017, SENASAG estimated more than 1,000 ha of crops had been affected and President Morales declared a state of emergency, dedicating \$700, 000 USD to control efforts (Luis Sanchez Shimura, SENASAG, pers. comm. to A. Cease; http://www.bbc.com/news/world-latin-america-38914922). The $S$. cancellata challenge is predicted to be ongoing and Bolivia, Paraguay, and Argentina are working together to develop a long-term research and management strategy.

\section{Evolution and diversity of swarming grasshoppers}

Out of 6,787 known species in the grasshopper family, the Acrididae (Cigliano et al., 2017), only 19 species are currently considered true locusts, which belong to at least four different subfamilies (Table 1; see also Pener and Simpson, 2009 (Table 1 therein); Song, 2011). It is clear from this spread across the Acrididae that swarming locusts have evolved multiple times but, at the same time, this has been a relatively rare occurrence (i.e. only $0.28 \%$ of known acridids are locusts). So, how did swarming locusts evolve and what are the factors promoting this transition? In order to address this question properly, it is important first to define what is a locust and how they differ from other grasshoppers. Pener (1983), defined locusts as acridids that (i) form dense migrating groups as bands of nymphs and/or swarms of adults, and (ii) are polyphenic in the sense that individuals living on their own or at low population densities differ in many characteristics from those living in groups. How rigidly demarcated is this definition?

Most of what we know about locusts comes from studies of two model species: The Desert Locust, Schistocerca gregaria and the Migratory Locust, Locusta migratoria (Pener and Simpson, 2009). However, not all locusts are similar to these two species because there are a large number of species-specific variations among "non-model" locust species (Song, 2011). For example, the Australian Spur-throated Locust, Austracris guttulosa and the Bombay Locust, Patanga succincta do not form nymphal bands (Song and Wenzel, 2008), while other locust species march as nymphs. Nymphal colouration of $C$. terminifera and Ca. italicus does not change in response to population density changes (Song, 2011), while density-dependent colour change is typically associated with true locusts. Moreover, there are some non-migratory grasshoppers that show a certain level of collective movement, such as Melanoplus sanguinipes and Camnula pellucida (Pfadt, 1988; Song, 
2011), as well as density-dependent colour change such as many species in the subfamily Cyrtacanthacridinae (Song and Wenzel, 2008; Figure 1). Furthermore, there are non-swarming species that are closely related to locusts, such as Schistocerca americana, which show densitydependent phenotypic plasticity reminiscent of true locusts (Sword, 2003; Gotham and Song, 2013) and exhibit a certain level of collective movement when the density is high enough (Kuitert and Connin, 1952). In other words, there is no clear-cut line between grasshoppers and locusts when we begin to examine the biology of acridids other than S. gregaria and L. migratoria. Instead, there seems to be a gradient of traits that range between 'sedentary' or 'non-migratory' grasshoppers and swarming locusts. Thus, to understand the evolution of swarming locusts, we need to examine the evolution of individual components that make up locust phase polyphenism, especially density-dependent phenotypic plasticity and collective movement. Furthermore, we need to approach these two phenomena from both process (i.e. adaptive significance) and pattern (i.e. phylogenetic) perspectives. When examined in detail, even species that show the full suite of traits associated with full locust phase polyphenism, such as S. gregaria and L. migratoria, differ markedly in their ecology, circumstances in which they undergo phase transition, rate of transformation and underlying physiological mechanisms, as set out in this review.

A defining feature of locusts is the expression of density-dependent phenotypic plasticity (Sword and Simpson, 2008; Simpson and Sword, 2009). While we often include colour, behaviour, morphometric ratios, and life history traits as major plastic reaction norms of locust phase polyphenism, it is now clear that not all of these reaction norms are plastic in all locust species (Song, 2011), and that different reaction norms of locust phase polyphenism have evolved separately from each other (Song, 2005; Song and Wenzel, 2008). In particular, density-dependent colour plasticity provides a good case study for how an individual reaction norm might have evolved. Initially, the function of density-dependent colour plasticity was unclear because the most obvious explanation - that the conspicuous colour of gregarious nymphs is aposematic - was not supported because vertebrate predators would readily feed on gregarious nymphs (Gillett and Gonta, 1978; Andiatsirevombola et al., 2016). A novel insight was provided by Sword (1999), who studied a Texas population of Schistocerca lineata, which feeds on a toxic plant (the Wafer Ash, Ptelea trifoliata). In this population, nymphs show density-dependent warning colouration, in which crowding induces bright yellow and black colour patterns in nymphs (Figure 1A), and it was experimentally shown that vertebrate predators quickly learn to associate this colour pattern 
with unpalatability. Based on this finding, Sword et al. (2000) tested whether a similar phenomenon occurred in $S$. gregaria and confirmed that, at the onset of population increase, gregarious $S$. gregaria nymphs in Mauritania preferentially feed on a native toxic plant, Egyptian Henbane (Hyoscyamus muticus: Solanaceae), which suggested that S. gregaria also expresses density-dependent warning colouration. More recently, Maeno et al. (2012) showed that solitarious nymphs of $S$. gregaria, which have a cryptic colouration ranging from green to brown, preferred to shelter on a spiny plant, Fagonia arabica, suggesting that solitarious nymphs use this plant as a physical protection against predators. These studies collectively suggest that density-dependent colour plasticity should be considered as an induced anti-predator strategy in both directions (Sword, 2002; Simpson and Sword, 2009). A recent study on the food preference of S. piceifrons showed that this species has a strong attraction to the odour of Pisonia aculeata, but has a feeding preference to two other plant species (Poot-Pech et al., 2016; see Section 10.3). Interestingly, $P$. aculeata is an evergreen thorn bush, which is used as a shelter or refuge. So, it is possible that further studies on $S$. piceifrons might uncover a similar pattern of behaviour to that observed in $S$. gregaria.

True aposematic warning patterns (as opposed to mimetic patterns) should signal that the organisms bearing them are toxic or unpalatable, which may be conferred either from ingested secondary plant compounds or by synthesizing defensive chemicals de novo. However, the feeding ecology of grasshoppers and locusts in relation to host plant toxicity is largely unknown for many species. It is also unclear why certain locust species do not show density-dependent colour plasticity (Song, 2011). From a phylogenetic standpoint, a plastic reaction norm in nymphal colouration should not be considered as an adaptation that has evolved de novo in locusts, but rather an exaptation. It is clear that density-dependent colour plasticity is a phylogenetically conserved trait, based on a broad survey within the Acrididae (Song, 2011), and at least within the Cyrtacanthacridinae (Figure 1; Song and Wenzel, 2008). Most species of Schistocerca, regardless of whether they are locusts or not, show this trait although the colouration induced by crowding does not appear to be classic warning colouration in many species (Song et al., 2017). More widely, it appears that many species within Cyrtacanthacrinae have the capacity to change colour in response to increased population density (Song and Wenzel, 2008; Song, 2011). Although it is still unclear how and why this plastic reaction norm evolved in the first place, its widespread expression suggests that it may not be costly to maintain or, at least, it is not strongly selected against. Thus, 
most Schistocerca species exhibit density-dependent colour plasticity not because it is adaptive in every species, but because their common ancestor already possessed this trait. A recent study showed that the Ptelea-feeding S. lineata, is a genetically distinct population within the species that has a wide distribution in North America (Raszick and Song, 2016). This suggests that this population was able to co-opt a pre-existing colour plasticity with an unknown function towards density-dependent warning colouration, as the population evolved a feeding preference to a toxic host plant. It is probable that a similar process has happened multiple times in various locust species, at least within the Cyrtacanthacridinae. See Figure 1 for some examples of colour polyphenism and polymorphism in the genus Schistocerca; the reader is referred to Pener and Simpson (2009) for detailed coverage of the physiological control of colour polyphenism.

There is some evidence that cannibalism may contribute to collective movement in nymphal bands of $S$. gregaria (Bazazi et al., 2008) and, in turn, nutritional state may influence the tendency to cannibalise. For example, Hansen et al. (2011) found that C. terminifera nymphs in a marching arena tended to cannibalise weak conspecifics, especially under conditions of protein deprivation (Hansen et al., 2011). Furthermore, individuals that cannibalised survived longer and thus moved farther than those individuals without the opportunity to cannibalise. In another study Bazazi et al. (2011) found that while nutritional state did not influence the movement of solitarious nymphs, feeding gregarious locusts a high carbohydrate, low protein diet led them to walk both more rapidly and more frequently (see Section 9 for more discussion on the influence of diet on phase change). More recently, Guttal et al. (2012) developed an evolutionary model, examining how the threat of cannibalism could influence the evolution of local interactions and collective movement in S. gregaria. Their model predicted that solitarious nymphs would show a clear avoidance of other individuals, but an increase in density will lead to a clear change in behaviour: an escape-pursuit behaviour in which locusts move away from nearing individuals, while at the same time pursuing individuals that are moving away. When simple avoidance behaviour does not inhibit conspecific interactions anymore, exploiting areas left vacant by other individuals is the most effective way to reduce encounters with conspecifics. This model shows that cannibalism could be one of the main drivers for the evolution of density-dependent behavioural plasticity (see Section 8.1 for further discussion on marching behaviour and the possible role of cannibalism).

A recent study showed that two non-swarming species of Schistocerca, S. americana and S. serialis cubense display density-dependent behavioural plasticity, although in a reduced form 
(Gotham and Song, 2013). This implies that plastic reaction norms in behaviour may still be retained in those species that are phylogenetically close to the locusts. In other non-migratory Schistocerca species however, crowding does not seem to induce any changes in behaviour, which suggests that behavioural plasticity has been lost in these species (Song et al., 2017).

Final insights into the evolution of swarming locusts come from population genetic studies of several locust species. Due to their migratory potential, locusts are generally expected to show a high degree of gene flow between populations (Chapuis et al., 2009, 2011). Indeed, recent studies were not able to find any major population genetic structures in several locust species, including solitarious populations of $S$. gregaria (Chapuis et al., 2014), C. terminifera (Chapuis et al., 2011), Ce. kiangsu (Fan et al., 2014), and the non-phase-changing but highly mobile pest species Oedaleus infernalis (Sun et al., 2015). Studies of the population genetic structures of L. migratoria showed that there are two major worldwide lineages, in contrast to a number of subspecies that previous taxonomic studies proposed (Tokuda et al., 2010; Ma et al., 2012). While each lineage covers a huge geographical area, the genetic diversity within seems to be minimal, a pattern that is consistent with other locust species. Another interesting finding comes from the comparison between swarming and non-swarming species. Blanchet et al. (2011) compared population genetic structure in three different species of the genus Calliptamus: the Italian Locust, Ca. italicus, even though it never swarms in the region where the study took place; Ca. barbarus, which has a similar wing length to $\mathrm{Ca}$. italicus; and $\mathrm{Ca}$. wattenwylianus, which has significantly shorter wings that result in a reduced mobility. As expected, $C a$. wattenwylianus showed a clear population genetic structure, even within a single field, but population genetic structure was absent in the other two species. Although most of these studies have relied on a relatively low number of molecular markers, they are consistent in highlighting the general pattern that there are very high heterozygosity values and/or high variance within populations, both of which are indicative of a large population size (Chapuis et al., 2008). It seems reasonable to postulate that where populations are this large and homogeneous, locust species are unlikely to speciate further because the lack of genetic structures and ecological differentiations among different populations would make it very difficult to develop reproductive isolation within species. The circumstances allowing for sympatric speciation or ecological speciation taking place in locusts would be exceptionally rare. The only way locusts could give rise to a new species is probably a founder event to an area where future gene flow would be significantly reduced. One such example is the southern subspecies of 
the Desert Locust, $S$. gregaria flaviventris. A recent study shows that this subspecies, which is restricted to the southwestern region of Africa, is genetically and morphologically divergent from the nominate subspecies in northern Africa (Chapuis et al., 2016). What is more intriguing is that this southern population has a much-reduced expression of density-dependent phenotypic plasticity (Waloff and Pedgley, 1986; Chapuis et al., 2017), which means that there could have been a selective pressure that promoted the loss of plastic reaction norms. When environmental conditions do not promote increases in population density and the expression of gregarious phase is not adaptive, perhaps because resources are homogeneously plentiful, locusts may give rise to non-swarming grasshoppers. A more clear case can be found in the wider Schistocerca genus, which contains approximately 50 species distributed throughout the New World (Song et al., 2013). It has been hypothesised that the North African $S$. gregaria is the earliest diverging lineage within the genus, and that the diversity of the genus in the New World is a case of adaptive radiation followed by multiple westward transatlantic colonizations by a "gregaria-like" ancestor (Lovejoy et al., 2006; Lorenz, 2009; Song et al., 2013). The fact that most Schistocerca species are non-swarming suggests that the genetic capacity to swarm that must have been present in the ancestral lineage has subsequently been selected against, suggesting that the environmental conditions in the New World must have been more favourable to cryptic and solitary life styles. The ability to swarm has eventually been lost, though remnants of density-dependent colour plasticity have remained.

In conclusion, we argue that the evolution of swarming locusts is a result of the evolution of numerous reaction norms that have followed different evolutionary trajectories throughout the diversification of grasshoppers. While we continue to gain an in-depth understanding of the proximate mechanisms of locust phase polyphenism from the studies of $S$. gregaria and $L$. migratoria, we should broaden our views to embrace a comparative perspective; there is clearly more than one way of becoming a locust.

\section{Measuring phase-related behaviour}

Laboratory-based experiments conducted since Pener and Simpson (2009) have largely continued to use variants of the assay system established by Roessingh et al. (1993) to quantify behavioural 
phase state. This assay seeks to condense the multidimensional nature of locust phase-related behaviour into a single metric of gregariousness (or solitariousness). Two broad categories of behaviour, relating to activity and attraction to other locusts, are particularly important in assessing phase state, both of which are much higher in long-term gregarious than in solitarious locusts. In setting up this assay in the laboratory a baseline of behavioural range and variation is established using observations of approximately 100 solitary-reared and 100 crowd-reared locusts, which are individually observed in a rectangular arena with a back-lit 'stimulus group' of 20-30 conspecific locusts behind a clear, perforated partition at one end and an empty back-lit chamber at the other end (discussed in detail in Simpson et al., 1999; Pener and Simpson, 2009 (p. 164); Cullen et al., 2012; Rogers et al., 2014). During each 5-10 min assay the observer records various behavioural measurements for the individual locust, which can be broadly divided into three categories: first, behaviours related to overall activity (e.g. distance moved, walking speed, time spent climbing the arena walls, ground covered/path tortuosity); second, behaviours related to overall attraction to, or repulsion from, the stimulus group (e.g. average distance to the stimulus group, time spent at the stimulus and/or non-stimulus end(s), or some composite thereof), and third, other small behaviours are sometimes included, particularly grooming time/frequency, which is consistently found to be higher in gregarious individuals of S. gregaria (e.g. Anstey et al., 2009; Ott et al., 2012; Rogers et al., 2014). Most studies of this nature have used immature locusts to avoid the added complications of flight and sexual behaviour that come with adult insects, though there have been some exceptions; see Cullen et al. (2012; Appendix Table A1 therein) for a comprehensive overview of all studies up to that year.

Following data collection for the 200 or more individual locusts of a known rearing history, the behavioural data for all insects are then fed into a stepwise binary logistic regression procedure, which selects the variables that best separate the average behavioural profiles of the two extreme phases before combining and weighting these variables to give the logistic equation. This equation can then be used to deliver a single value of an individual locust's behavioural state. With the baseline behavioural variation of the two phases established, locusts of unknown phase state can then be tested in the arena assay with reference to these two model populations. When designated as $P_{\text {greg }}$ (i.e. the probability of being in the gregarious phase) a returned value close to 1 means that the behaviour shown by the test locust is much more likely to be observed among long-term gregarious locusts, while a $P_{\text {greg }}$ close to 0 means that the behaviour of the test animal is much 
more similar to that seen in long-term solitarious locusts (or vice versa where the inverse ' $P_{\text {sol', }}$ ', i.e. probability of being in the solitarious phase, is used instead). Any subsequent test locust that has been subject to some experimental manipulation (e.g. specific sensory stimulation, pharmacological treatment, RNA interference, nutritional perturbation) is then assayed in the same way, with a value of $P_{\text {greg }}$ returned as a single composite predictor of that animal's behavioural phase state.

\subsection{RECENT BEHAVIOURAL STUDIES}

\subsubsection{Behavioural assays of Schistocerca gregaria}

Notable studies that have implemented the Roessingh et al. (1993) assay system over the past decade include the neuropharmacological work of Anstey et al. (2009) and Ott et al. (2012), which respectively showed that serotonin (5HT) and Protein Kinase A (PKA), a downstream effector of several biogenic amine receptors, are both necessary and (in the case of 5HT) sufficient to trigger the transition towards gregarious behaviour in S. gregaria. The behavioural models in both of these studies correctly classified $90 \%$ of the locusts from the model-building population, and featured almost identical variables for returning the final measurements of $P_{\text {greg }}$; walking speed, proportion of time at rest, and time spent in the $25 \%$ of the arena next to the stimulus group were used in both cases. The fourth variable in both models was grooming, but measured in slightly different ways in each case (total grooming time in Anstey et al. (2009), and frequency of grooming episodes in Ott et al. (2012)). The specific weightings for each variable also differed marginally between the two studies, which is unsurprising given that the two models were built using locusts from different laboratory colonies (the former Oxford University S. gregaria colony for Anstey et al. (2009), and the Cambridge University S. gregaria colony for Ott et al. (2012)). The fact that such similar behavioural models could be independently applied to distinct colonies of $S$. gregaria stands as a testament to both the robustness of the Roessingh et al. (1993) assay and the $P_{\text {greg }}$ behavioural metric, and to the reliability with which $S$. gregaria will generally perform in the relatively unnatural setting of a rectangular arena-based assay.

The four individual behaviours retained in these models change in concert throughout the course of behavioural phase transition, as demonstrated more recently in a meta-analysis of $S$. 
gregaria behavioural transition by Rogers et al. (2014). This study deconstructed $P_{\text {greg }}$ into its constituent behavioural components, using data collected across an 8-year period of research (Simpson et al., 2001; Rogers et al., 2003; Anstey et al., 2009), and supported previous findings that forced crowding, tactile stimulation of the hind femora, and the pharmacological application of serotonin all induce synchronous changes in the full suite of retained behavioural measurements including grooming and attraction to other locusts, and not merely changes in activity (as had been suggested by Tanaka and Nishide, 2013). Furthermore, and importantly, Rogers et al. (2014) also showed that no single behavioural variable retained in the model was the sole driver of $P_{\text {greg }}$, and no single variable changed disproportionately strongly relative to the others in the face of serotonergic driving of behavioural phase. Indeed, across the 196 solitarious and 196 gregarious locusts assayed throughout the three studies considered in the meta-analysis, not a single locust with a $P_{\text {greg }}$ below 0.43 in the composite behavioural model approached the stimulus group of conspecific locusts and, conversely, only two locusts with a $P_{\text {greg }}$ above 0.8 did not approach the stimulus group. This study serves to highlight the necessity of a multidimensional behavioural model, in which measures of both activity and group-related behaviours are important for producing a well-balanced predictor of phase state.

A different methodology for measuring behavioural phase change in S. gregaria was taken by Geva et al. (2010). Instead of separate crowding treatments and assay measurements, test solitarious and gregarious $4^{\text {th }}$ instar locusts were placed in $20 \mathrm{~cm}$ diameter circular arenas and crowded with ten long-term gregarious locusts and continuously filmed. From these videos 5-min bins were extracted for quantitative behavioural analysis. Solitarious locusts, on being placed in a crowd from which they could not remove themselves, were highly aroused and exhibited high activity, jumping, and avoidance behaviour as they sought to escape. By contrast, gregarious locusts were much less active in this behavioural context. This situation is therefore the opposite to that seen during the Roessingh et al. (1993) style behavioural assays, in which stimulus and testing regimes are separated, and where solitarious locusts are much less active than gregarious locusts. The overall activity of solitarious locusts decreased rapidly within $30 \mathrm{~min}$ as they remained in the crowd. A logistic regression model using the activity of solitarious and gregarious during the first 5 min of crowding as the model populations was constructed. The model showed some convergence in $P_{\text {sol }}$ over the course of $4 \mathrm{~h}$ of crowding, driven principally by a dramatic change in the solitarious locusts, but the gregarious locusts also changed from their baseline, presumably as 
they became more active following prolonged crowding. The authors interpret these data as demonstrating a behavioural phase change process and that habituation to the presence of other locusts is an important element driving behavioural gregarisation. Solitarious locusts crowded for $30 \mathrm{~min}$, removed from the crowd for $24 \mathrm{~h}$ and then reintroduced for a further $30 \mathrm{~min}$ retained a memory of their prior exposure and were less active on re-introduction to the crowd than naïve locusts, or even locusts that had been continuously crowded for an hour. This long-lasting effect could be removed by treating the locusts with the protein-synthesis inhibitor anisomycin one hour after a 30 min crowding session; on re-introduction into the crowd, locusts displayed the high activity characteristic of naïve locusts.

It is unclear whether the behaviour highlighted by Geva et al. (2010) is the same kind of behavioural change seen using other experimental paradigms. At the neuronal level, several studies have shown that solitarious locusts undergo pronounced habituation to repeated visual looming stimuli (Matheson et al., 2004; Rogers et al., 2007, 2010) and to tactile stimulation of the head (Fuchs et al., 2003), whereas habituation is much more muted in gregarious locusts (see Section 7). Of itself therefore, habituation is not evidence of gregarisation. Other experiments suggest that gregarisation, or at least some aspects of it, is not very habituation-like. For example, locusts subjected to tactile stimulation of the hind femora for $4 \mathrm{~h}$ (Simpson et al., 2001; see Section 4 below) behave in a manner identical to long-term gregarious locusts even though they have never had any exposure to other locusts. Furthermore, the sensory modality used to engender the behavioural change, touch, is not available in the Roessingh style arena, in which the locusts can see and smell other locusts in the stimulus group but not touch them; there is a complete disjunction between the stimuli inducing gregarisation and those available during the testing of it. A general loss of arousal during prolonged tactile stimulation can be excluded because of the specific nature of the tactile stimulus needed to evoke gregarisation, and by the general increase of activity characteristic of the gregarious phase seen in this behavioural context. Nevertheless, the rapid behavioural habituation and loss of arousal seen by solitarious locusts when placed in a crowd may be an important means of capturing and retaining them within a group so that they are subjected to prolonged exposure to gregarising stimuli.

\subsubsection{Automated video tracking of Chortoicetes terminifera}


Despite the consistently robust behavioural models that the Roessingh et al. (1993) assay and logistic regression have returned for $S$. gregaria, the observer-dependent nature of this manual assay system has historically meant that each researcher has had to build their own model from an independent set of solitarious and gregarious locusts. This has inevitably made it difficult to directly compare the raw behavioural data (and their resultant $P_{\text {greg }}$ values) between studies, particularly those conducted in different research groups, since they were each based on measurements by different human observers, and therefore potentially subject to inherent observer-based variation. This has arguably led to the unfortunate situation in which each study (or laboratory-linked cohort of studies) of behavioural phase using the Roessingh et al. (1993) assay (or indeed, any other observer-dependent system) has tended to stand alone as a discrete set of experiments with only a limited capacity for direct comparison between datasets. This applies to both the baseline behaviour of the model-building gregarious and solitarious locust populations, and the experimental/transitional animals between the two extremes.

Fortunately, these issues should be resolved with the increasing implementation of automated video tracking to the Roessingh et al. (1993) assay system. This development is among the most significant advances in methodology that has occurred in locust behavioural phase research in recent years, with the first study to do so (Gray et al., 2009) the only one covered by Pener and Simpson (2009). Gray et al. (2009) used the commercial software EthoVision ${ }^{\circledR} 3.1$ (Noldus Information Technology, Wageningen, The Netherlands; Noldus et al., 2001) to address the hitherto unresolved question of whether the Australian Plague Locust, Chortoicetes terminifera, expresses behavioural phase polyphenism. EthoVision, which was originally developed for studies in rodents, uses a proprietary video tracking algorithm to return a suite of pre-programmed behavioural measurements, independent from the operator and therefore repeatable. Crucially, these include measurements from the two main categories of phase-related behaviour; activity (including distance moved, velocity and several measures of path tortuosity, all relative to a ruler on the calibration image taken with the recording camera) and position relative to the stimulus group (as defined by zones drawn by the user on a calibration image within the graphical user interface). Gray et al. (2009) definitively showed that C. terminifera expresses strong behavioural phase polyphenism. The logistic regression model built from EthoVision output data of final-instar nymphs retained total distance moved (activity-related), average distance to the stimulus chamber (position to conspecifics-related) and total time climbing the arena walls (a 
mixture of activity and positional data) to correctly classify $97.4 \%$ of the 78 crowd-reared model insects, and $100 \%$ of the 78 solitary-reared locusts. This overall accuracy of $98.7 \%$ remains the highest yet published for a locust behavioural model, and demonstrates both the power of automated video tracking and the robust expression of behavioural phase polyphenism in $C$. terminifera. Automated video tracking also confers the added advantage that multiple behavioural arenas can be tracked at the same time (two in the case of $C$. terminifera), speeding the conduct of laboratory-based experiments.

Following on from Gray et al. (2009), further behavioural work in final-instar $C$. terminifera used automated video tracking to determine the proximal stimuli that induce phase change in this species. Cullen et al. (2010) tested the effects of olfactory and visual stimuli (both in isolation and in combination) and the effects of tactile stimulation directed to a range of body parts on phase transition in C. terminifera, in an experimental design similar to those in previous studies of S. gregaria (Simpson et al., 2001; Rogers et al., 2003). Cullen et al. (2010) determined that tactile stimulation of the antennae is the key stimulus triggering behavioural phase change in C. terminifera; see Section 4 for further discussion. Furthermore, Cullen et al. (2010) extended the methodology for automated behavioural tracking in $C$. terminifera by employing an identical EthoVision-based assay to Gray et al. (2009) but using a two-step model building and validation process. In the first step, a behavioural model was built using tracking data for $70 \%$ of the available model population (amounting to 98 solitarious and 75 gregarious locusts in this subset). The most accurate logistic regression model correctly classified $93.9 \%$ of the solitarious and $90.7 \%$ of the gregarious insects, and retained four of the available behavioural variables incorporating both activity- and position-based data; mean angular velocity, mean velocity, time spent in the nonstimulus third of the arena, and time spent climbing the non-stimulus wall. In the second step, this model was validated by testing it against the remaining $30 \%$ of the available model population ( 25 solitarious and 49 gregarious locusts). The model correctly classified $96 \%$ of the solitarious and $97.9 \%$ of the gregarious locusts in the validation subset, giving an overall accuracy of 93.9\% (not $92.5 \%$ as the authors erroneously conclude in their discussion). This simple 70:30 division of the available model population into separate model-building and model verification cohorts ensured that the model was not over-fitted to the behaviours of the $70 \%$ cohort from which the model was built but had general applicability, and guaranteed the robust downstream assaying of experimentally manipulated locusts in the same study. Cullen et al. (2012) performed further 
optimisation experiments for the automated video tracking of $C$. terminifera final-instar nymphs, with a focus on ascertaining the most biologically pertinent behavioural variables to include in the model-building process. They showed that climbing variables should be treated with caution, as they were essentially redundant 'edge effect' measurements of the times spent in the stimulus and non-stimulus zones. This study also determined the optimum assay duration, by building models from the data extracted from pre-recorded behavioural tracks that were arrested after the first 2, 4, 6 or $8 \mathrm{~min}$. The only robust model, which correctly classified $92.4 \%$ of the 251 model insects, was built from the streamlined suite of behavioural variables for the full 8-min tracks; all other models were substandard in some way. Finally, and perhaps surprisingly, Cullen et al. (2012) also showed that automated video tracking is capable of extracting behavioural data that were previously unnoticed, or otherwise unquantified. 'Mean angular velocity' varied significantly between gregarious and solitarious $C$. terminifera, and upon closer inspection of the raw video tracks the authors realised that this was due to a greater number of small turns and body movements in the solitarious animals, which were typically performed on the non-stimulus side of the tracking arena. This variable, which would have gone unnoticed without automated video tracking, was retained in their optimal behavioural model. Conversely, thus far, automated tracking has not been successful in picking up some of the discrete behavioural differences between solitarious and gregarious locusts that were a feature of some of the manual models. These behaviours include grooming, which is consistently higher in gregarious S. gregaria (Rogers et al., 2014), and peering,

in which locusts move the head side-to-side to estimate distance from motion parallax cues (Collett, 1978). Although EthoVision is currently the de-facto standard for automated video tracking in locust phase research, a freely available open-source software alternative has been developed and used successfully in S. gregaria (Stettin and Ott, 2012; Stettin, 2015).

\subsubsection{Automated video tracking of Locusta migratoria}

The Roessingh et al. (1993) assay system augmented with automated video tracking by EthoVision has also been successfully employed across a program of phase-related research in L. migratoria, to unravel the molecular physiology of behavioural phase in $4^{\text {th }}$ instar nymphs (discussed at length in Section 5.2). The first study to perform automated video tracking in L. migratoria (Ma et al., 2011) implicated dopamine synthesis and release as the triggers for phase change in this species, 
and started by attempting to establish separate logistic regression models for each of the five larval instars. The stepwise model selection retained different subsets of behavioural predictor variables for different instars, but this does not necessarily indicate that behavioural phase differences differ between instars. Instead, the different sets of retained variables may at least in part be coincidental outcomes that reflect the instability of stepwise variable selection in the presence of correlations among predictors (Harrell, 2001). Logistic regression models for the $1^{\text {st }}$ and $2^{\text {nd }}$ instar nymphs of L. migratoria failed to retain any positional variables and, in the case of the $1^{\text {st }}$ instar assay, the two retained variables were likely measurements of the same thing (total distance moved and frequency of movement). The authors describe these first two instars as exhibiting "partial phasespecific behaviour", but basing this conclusion on stepwise regression alone is potentially problematic. These early instars of L. migratoria may indeed have lacked any phase-related differences in attraction to conspecifics. Alternatively, such differences may have merely eluded detection due to the particular variables and arena zones stipulated by the authors, or due to the shortcomings of automated stepwise model building. Models for the $3^{\text {rd }}, 4^{\text {th }}$ and $5^{\text {th }}$ instars successfully retained measurements of both activity and position relative to the stimulus group, but again with an apparent degree of redundancy. Alongside frequency of movement, the $3^{\text {rd }}$ instar model retained entry frequency into the $25 \%$ of the arena near the stimulus zone and total duration close to walls, though the particular walls were not specified so it is possible that both variables were measurements of the locusts' movements near the stimulus group. Furthermore, entry frequency into the stimulus zone (or any other zone) is also highly likely to correlate with activity; a locust walking in rings around the outside of the arena will enter zones more frequently than a locust that has approached the stimulus zone and then remained there for the duration of the assay. The $4^{\text {th }}$ instar model, by contrast, only retained one position-related variable ('attraction index', a simple composite of the time spent in the $25 \%$ of the arena near the stimulus group, minus the time spent in the opposite non-stimulus end) but two similar measurements of activity (total distance moved and total duration of movement). Accuracies of these behavioural models were not given, though an almost identical version of the $4^{\text {th }}$ instar L. migratoria model was used in Guo et al. (2011), in which the authors implicate the olfactory-related chemosensory protein and takeout genes in phase-related attraction/repulsion behaviour of this species. This model combined the same three behavioural variables (total distance moved, total duration of movement and attraction index) with almost identical weightings in the model, to return an average accuracy of $87.1 \%$ 
(correctly classifying $87.2 \%$ of 86 gregarious model locusts and $87 \%$ of 69 solitarious animals). Further evidence of variable redundancy is evident in Tong et al. (2015), who again use a model retaining the same three variables but also present plots for each of them separately (see Fig. 4 therein). Their bar charts demonstrating the separation between solitarious and gregarious $L$. migratoria for total distance moved and total duration of movement are highly similar, suggesting that one variable is probably redundant. More recently, this canon of work in L. migratoria has settled on a common model in three studies investigating the role of biogenic amines in behavioural phase in this species (Guo et al., 2013; Ma et al., 2015; Xu et al., 2017). Again focussing on $4^{\text {th }}$ instar nymphs, this model achieved a slightly higher accuracy of $90.2 \%$ (89.2\% of 100 solitarious model locusts and $91.2 \%$ of 100 gregarious animals) but also contains a potential redundancy among the two activity-related variables, total distance moved and frequency of movement, retained alongside attraction index.

The behavioural models for L. migratoria each contain at least one activity- and one position-related variable, thereby capturing some element of the multidimensional nature of locust behavioural phase. Nevertheless, there does appear to be some redundancy in each of the logistic regression models highlighted above. Total distance moved, total duration of movement, and frequency of movement are highly likely to be significantly correlated and, without access to the raw data (as is usual), it is impossible to determine the extent to which these separate measures of activity add some additional behavioural information into their respective models, if any. Furthermore, the accuracies of these models (where given) are not as uniformly high as they have been for those of S. gregaria (e.g. Simpson et al., 2001; Rogers et al., 2003; Roessingh et al., 1998) or more recently for $C$. terminifera, which were also obtained using automated video tracking (Gray et al., 2009; Cullen et al., 2010, 2012). Nevertheless, the recent behavioural models for $L$. migratoria obtained through automated tracking have still been a substantial step forward since, prior to the work discussed here, there were few studies in which L. migratoria were assayed in this type of arena at all. In one notable exception, Hoste et al. (2002) assessed the phase-related behaviour of an albino strain of L. migratoria deficient in the neuropeptide [His ${ }^{7}$ ]-corazonin. Their model, which retained turn rate, single leg movement count and time in the non-stimulus third of the arena, only achieved an accuracy of $70.2 \%$ (a value of $50 \%$ would be obtained by random guessing). 
We finish this section by suggesting a small number of recommendations with a view to obtaining some universally recognised standards in automated video tracking that capture the multidimensional nature of locust behavioural phase: (i) Wherever possible, try to ensure that models retain both activity- and position-related data. (ii) When selecting behavioural variables to input into the behavioural model (either logistic regression-based or otherwise), choose only those that have the potential to add some extra information to the model; therefore, avoid using redundant measurements of the same behaviour (e.g. total distance moved, walking duration, and number of walking bouts). Ensure that climbing does not correlate strongly with some other activity- or position-related variable, and that frequency of zone entry is not simply a redundant measurement of activity. (iii) Where possible, verify the accuracy of the model with a separate subset of behavioural data, obtained from animals with a known history. Perform formal tests of model fit wherever possible (see those discussed in Hosmer et al. (1997) for logistic regression). (iv) Finally, present the raw data for each of the variables retained in the model, and plot as separate graphs (e.g. in the Supplementary Data for the publication) to allow scrutiny of how specific behavioural changes and their intercorrelation lead to an altered $P_{\text {greg. }}$. This final point has become increasingly important as more strains and species of locusts are assessed for behavioural phase change. The logistic regression approach is bounded by the variation shown by the model populations used to construct each model. It cannot meaningfully compare the variation size between solitarious and gregarious locusts across different strains or species. For example, a 'strong' classification may be achieved between 'solitarious' and 'gregarious' populations with little absolute magnitude of difference between them if the variance of each model population is small. Conversely, model populations where the average difference is large may not be easily classified if the behavioural characteristics within the solitarious and gregarious groups all have large variances. Presently the only way to compare between different solitarious and gregarious populations across laboratories, strains or species is to look at the actual magnitude of behavioural difference using external measurement criteria (e.g. path length in $\mathrm{cm}$ ), rather than the internal yardstick provided by $P_{g r e g}$. A further reason for presenting data for individual behaviours is that it helps assess whether an experimental treatment affects all aspects of locust behavioural phase, or just particular 
behavioural components. This has the potential to greatly assist in our understanding of the mechanistic basis of behavioural phase change, and its evolution in different species of locust.

\section{$4 \quad$ Proximal stimuli inducing phase change}

Prior to Pener and Simpson (2009), the proximal stimuli that induce behavioural phase change were far better understood in S. gregaria than in any other locust species. Roessingh et al. (1998) showed that solitarious $S$. gregaria nymphs underwent full behavioural gregarisation within $4 \mathrm{~h}$ when presented with combined visual and olfactory stimuli from a group of gregarious nymphs, but neither visual nor olfactory stimulation on its own was effective. The same study also indicated that mechanosensory stimulation was a potent gregarising stimulus, as solitarious nymphs gently buffeted with papier maché spheres for $4 \mathrm{~h}$ also subsequently exhibited fully gregarious behaviour. This mechanosensory stimulus was localised to the hind femora by Simpson et al. (2001), in which various body parts were each stroked with a paintbrush for $5 \mathrm{~s}$ each minute over a $4 \mathrm{~h}$ period, and yet further still by Rogers et al. (2003), who showed that stroking half of the femur produced a stronger shift in behavioural phase than that produced by only stroking one quarter. Importantly, Rogers et al. (2003) also showed that restraining the femur relative to the thorax did not lead to behavioural gregarisation with subsequent paintbrush tickling, demonstrating that both mechanosensory from the hind femora and proprioceptive stimuli from the proximal leg joints are required to induce behavioural gregarisation in S. gregaria through this pathway.

More recently, progress has been made identifying the proximal stimuli that induce behavioural gregarisation in C. terminifera and L. migratoria. Cullen et al. (2010) emulated the experimental design of Roessingh et al. (1998) and Simpson et al. (2001) in a behavioural study of $C$. terminifera. After $6 \mathrm{~h}$ of treatment, the only stimulus that triggered significant gregariousness in C. terminifera final-instar nymphs was tactile stimulation of the head (including antennae) or the antennae in isolation. Tactile stimulation of the abdomen also produced a shift towards gregarious behaviour that was near significant, but the authors showed in a separate experiment that locusts tickled on the abdomen jumped over 17 times more frequently than those tickled on the antennae during their respective treatments (1151 versus 66 jumps between 10 locusts over 6 $h$, respectively). The authors therefore concluded that the apparent effect of abdominal 
mechanostimulation was likely due to these locusts self-stimulating their antennae as they jumped against the inside walls of their holding chambers (Cullen et al., 2010; Fig. 3 therein). It is important to note that the only previous paper to investigate behavioural gregarisation in $C$. terminifera (Gray et al., 2009) used $72 \mathrm{~h}$ of crowding with conspecifics as its shortest period of stimulation. Behavioural gregarisation in C. terminifera was achieved after $6 \mathrm{~h}$ of tactile stimulation, which suggests that much shorter periods of crowding should also be sufficient to induce phase change in this species, though this has yet to be reported.

Equivalent experiments to identify potential gregarising stimuli in L. migratoria have yet to be reported. However, recent experiments in L. migratoria tentatively suggest a role of olfaction during the process of phase change. Guo et al. (2011) used microarrays and qPCR to demonstrate a strong differential expression in two chemosensory-related gene families between antennal tissues of the two phases. Several chemosensory protein (CSP) transcripts showed a significantly higher expression in the antennae of gregarious nymphs than in the antennae of solitarious nymphs, and knockdown of one of these (CSP3) with RNAi caused a significant repulsion from the stimulus group in their behavioural assay, as measured by an attraction index (Guo et al., 2011; Fig. 5 therein). As the authors point out, attraction to the stimulus group in the Roessingh et al. (1993) assay could be due to either visual or olfactory attraction, or both, so Guo et al. (2011) also performed a separate experiment to test for olfactory attraction/repulsion using a simple Y-maze choice assay, in which nymphs walking through a plastic tube could enter an arm drawing through clean air, or an arm drawing through volatiles from 30 conspecific nymphs. This assay confirmed the authors' findings from the arena assay; gregarious nymphs treated with RNAi against CSP3 significantly reduced their preference for the volatile-containing arm, opting instead to follow the clean air. By contrast, one takeout gene showed a significantly higher expression in the antennae of solitarious than in gregarious nymphs, and RNAi of takeout in solitarious animals produced the opposite effect; they showed a significantly increased attraction to the stimulus group in the behavioural assay, and a significantly decreased aversion to locust volatiles in the Y-maze.

An important observation by Guo et al. (2011) is that, despite the clear effects of CSP3RNAi on olfaction-led attraction behaviour in gregarious L. migratoria, and of takeout-RNAi on olfaction-led repulsion behaviour in their solitarious counterparts, neither treatment influenced activity levels as measured in the arena assay (see their Fig. 5 and Discussion). Furthermore, a time-course of forced crowding in the early stages of this study showed that solitarious nymphs 
were still significantly behaviourally distinguishable from the gregarious model population after $64 \mathrm{~h}$ of crowding. The authors attribute this relatively slow shift in $P_{\text {greg }}$ to an asynchronous timecourse of changes in the component behaviours of their model; attraction index increased steadily with forced crowding, while the two activity-related variables (total distance moved and total duration of movement) took $32 \mathrm{~h}$ of crowding before they significantly increased (see their Fig. 1 for $P_{\text {greg }}$ and Supplementary Fig. 1 for the component behaviours). The same trend is demonstrated in Ma et al. (2015), in which similar data for attraction index and Y-maze assays show a steady increase in olfactory-based attraction behaviour but without a corresponding change in activity until at least $32 \mathrm{~h}$ of crowding. The fact that these experimental L. migratoria were still distinguishable from the long-term model building populations in both Guo et al. (2011) and Ma et al. (2015) means that they were, by definition, still in the transitional stage between solitarious and gregarious. Coupled with the apparent importance of olfactory-led attraction during this period, it seems very likely that olfaction plays a key role in inducing phase change in $L$. migratoria. Nevertheless, visual and tactile stimuli should also be tested, both in isolation and in interaction with the olfactory stimulus, to unravel the full suite of stimuli capable of causing gregarisation in this species.

Stimuli inducing phase change in C. terminifera and L. migratoria are both potentially mediated via the antennae. Furthermore, both species are members of the family Oedipodinae, and are therefore more closely related to each other than either species is to S. gregaria (a member of the Cyrtacanthacridinae), in which phase change is induced by quite different mechanisms (the olfactory route in this species is in interaction with a visual stimulus). It could be speculated that L. migratoria and C. terminifera share a common mechanism of behavioural phase change, which has been overlooked due to the different methods used to assess the two species. In the case of $C$. terminifera, nymphs of this species do not tend to engage in prolonged tactile interaction in laboratory culture (Cullen, unpublished observations), so the precise nature of tactile stimulation as demonstrated by Cullen et al. (2010), and how it relates to natural populations of C. terminifera, is yet to be determined. Even so, Cullen et al. (2010) also showed beyond doubt that olfactory stimuli did not induce behavioural phase change in this species; the olfactory stimulus (the volatiles from 50 conspecifics for $6 \mathrm{~h}$ ) produced a median $P_{\text {greg }}$ of 0.0638 , the lowest of all the stimuli tested. It should also be noted that the stimuli that induce gregarisation need not necessarily be the same as the stimuli that maintain gregarious behaviour, and future experiments should also 
seek to identify the minimum stimuli required for maintaining gregarious behaviour in both $L$. migratoria and $C$. terminifera, with a view to making a comprehensive comparison between these two species and the more distantly related S. gregaria. As more species of Acridid become identified as undergoing full or partial density-dependent behavioural polyphenism (see Section 2) it is possible that the range of mechanisms and sensory modalities that mediate gregarisation will expand, reflecting the independent evolution of phase change across several different lineages.

\section{$5 \quad$ Biogenic amine signalling}

The biogenic amines serotonin, dopamine, octopamine and tyramine are implicated in behavioural phase change in two well-studied locust species: S. gregaria and L. migratoria. In this section, we first introduce the different receptor classes found in insects and review the likely efficacy and specificity of different pharmaceutical agonists and antagonists when used on locusts. Second, we provide an overview of our current understanding of the role of biogenic amines and their receptors in locust phase transition.

\subsection{PHYLOGENY AND PHARMACOLOGY OF BIOGENIC AMINE RECEPTORS}

The biogenic amines are central to many cellular signalling networks underlying plasticity by inducing transient changes in intracellular second messengers. These regulate a wide range of kinase enzymes, which change target protein function by altering their activity, cellular location and/or interaction with other proteins. Biogenic amines thus lead to transient functional changes of target proteins and, in the longer-term, regulate transcription in the cell nucleus. They are closely associated with a wide variety of insect behaviours (Libersat and Pflueger, 2004; Blenau and Bauman, 2001; Verlinden et al., 2014).

In insects, biogenic amines can be divided in the indolamine serotonin (5hydroxytryptamine or 5-HT; derived from tryptophan), the catecholamine dopamine (derived from tyrosine via L-DOPA), the monophenolic amines octopamine and tyramine (also derived from tyrosine) and the imidazolamine histamine (derived from histidine). Serotonin and dopamine occur universally across the animal kingdom, where they are involved in major physiological processes 
with many conserved functions. Octopamine and tyramine are the invertebrate counterparts of noradrenaline and adrenaline respectively (Pflüger and Stevenson, 2005), but only occur in trace amounts in vertebrates. Histamine is also present in both vertebrates and invertebrates, but whereas most signalling in vertebrates takes place through G-protein coupled receptors (GPCRs), in invertebrates all known histamine signalling is mediated by ligand-gated ion channels (Fleck et al., 2012; Kita et al., 2016). In general, most biogenic amine receptors are GPCRs, belonging to the rhodopsin GPCR family. However, several amines can also interact with specific ligand-gated ion channels.

\subsubsection{Phylogeny of the biogenic amine receptor families}

If all the sequences of biogenic amine GPCRs are placed together in a phylogenetic tree, it is clear that the different receptor classes for each of the biogenic amines have common origins, rather than being linked most closely by the biogenic amine ligands themselves (Hauser et al., 2006; Hauser et al., 2008; Spielman et al., 2015; Qi et al., 2014; Wu et al., 2014). This may indicate that a hypothetical ancient signalling molecule had a range of receptor classes, each coupled with different downstream signalling pathways. Slightly different signalling molecules then evolved, either by evolutionary drift or by a selected for process, each with their own set of associated receptors. Most of the biogenic amine receptors can be activated by high doses of other biogenic amines, which supports this hypothesis. Another explanation for the observation that biogenic amine receptors do not cluster based on ligand-binding could be that these receptors have undergone extensive functional convergent evolution, which seems less plausible. Whatever their exact origin, it is certain that the major classes of biogenic amine receptors arose before the divergence of protostomes and deuterostomes. Subsequent gene duplications and structural/functional differentiation occurred generating further subtypes within each main receptor class. These receptor subtypes are believed to have evolved independently in vertebrates and invertebrates (Peroutka and Howell, 1994; Tierney, 2001; Vleugels et al., 2015). During this

evolutionary process, selection would have been based on functionally important receptor characteristics such as ligand binding and coupling to second messengers, not the conservation of recognition sites for synthetic pharmaceutical ligands (Vleugels et al., 2014). Hence, as yet there is no general pharmacological classification system available for invertebrate biogenic amine 
receptors, which needs careful consideration when designing experiments. Most pharmaceutical agonists and antagonists are classified purely on their effects on mammalian biogenic amine receptors, and there is no guarantee that such agents will affect insect receptors in a similar manner, if indeed at all.

Some general findings are listed below, although it should be noted, firstly, that not all chemicals were tested on all types of receptor; secondly, that cell assay-specific differences have been observed for some receptors; and thirdly, that putative species-specific differences should also be considered. Furthermore, no direct measurement of the binding of pharmaceutical agents onto specific $\mathrm{G}$ proteins in insects has been performed so far; instead, indirect measures using secondary messenger molecules have been used.

\subsubsection{Serotonin receptors}

In vertebrates, 5-HT receptors are divided in seven classes. All but 5- $\mathrm{HT}_{3}$, a ligand-gated ion channel, are GPCRs. 5- $\mathrm{HT}_{1}$ and 5- $\mathrm{HT}_{5}$ receptors couple to $\mathrm{G}_{\mathrm{i} / \mathrm{o}}$ proteins and thus reduce cAMP synthesis in the target cells. 5- $\mathrm{HT}_{2}$ receptors preferentially couple to $\mathrm{G}_{\mathrm{q} / 11}$ proteins leading to increase of intracellular calcium levels. 5- $\mathrm{HT}_{4}, 5-\mathrm{HT}_{6}$ and $5-\mathrm{HT}_{7}$ receptors all bind to $\mathrm{G}_{\mathrm{s}}$ proteins, increasing intracellular cAMP production (Nichols and Nichols, 2008).

Thus far, six types of 5-HT receptor belonging to four classes have been characterised in insects.

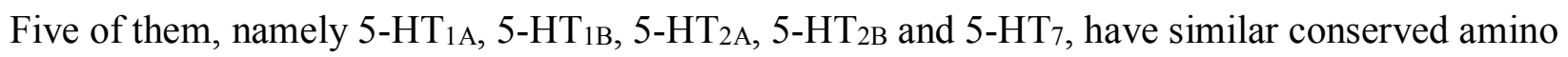
acid sequences and activate the same second-messenger systems as the equivalently numbered vertebrate classes (Colas et al., 1995; Röser et al., 2012; Saudou et al., 1992; Schlenstedt et al., 2006; Thamm et al., 2010; Troppmann et al., 2010; Vleugels et al., 2013; Vleugels et al., 2014; Witz et al., 1990). The sixth type is an invertebrate-specific 5-HT8 receptor, which shows less sequence conservation to any other receptor subtype (Qi et al., 2014). It mediates increases in intracellular calcium levels and does not affect intracellular cAMP levels (Qi et al., 2014).

Agonists (excluding naturally occurring biogenic amines) able to activate all tested invertebrate 5-HT receptors when applied in the micromolar range are 5-methoxytryptamine, $( \pm)$ 8-hydroxy-2-(di-propylamino) tetralin (8-OH-DPAT), 5-carboxamidotryptamine (5-CT) and possibly also $\alpha$-methyl 5-hydroxytryptamine ( $\alpha$-Me5-HT), although this has not been tested on 5HT8 receptors. ( \pm )-1-(2,5-dimethoxy-4-iodophenyl)-2-aminopropane ( $\pm-D O I)$ is a potent 
hallucinogenic drug in humans that is a highly selective agonist of the mammalian 5-HT2 receptor family. In insects this also seems to be the case, although it has only been tested on Drosophila 5$\mathrm{HT}_{1 \mathrm{~A}}$ and 5-HT2 type receptors (Colas et al., 1995; Johnson et al., 2009).

Prazosin, dihydroergocryptine, 1-butaclamol and d-blutaclamol may be good antagonists for $5-\mathrm{HT}_{1}$ and $5-\mathrm{HT}_{7}$ receptors, while $5-\mathrm{HT}_{2}$ receptors can be antagonised by clozapine and cyproheptadine. WAY-100635 inhibits 5- $\mathrm{HT}_{1}, 5-\mathrm{HT}_{7}$ and 5- $\mathrm{HT}_{8}$ receptors; while ketanserin inhibits 5-HT2 and 5-HT7 receptors (Colas et al., 1995; Thamm et al., 2013; Vleugels et al., 2014; Qi et al., 2014; for an overview see Vleugels et al., 2015). Methysergide is a non-selective 5-HT receptor antagonist, but was not tested on 5-HT8. In addition, in the blowfly, Calliphora vicina, methysergide activates 5-HT7 but inhibits 5-HT2A-mediated responses (Röser et al., 2012). In the honey bee, Apis mellifera, methysergide displays high potency at 5-HT2A but does not block 5$\mathrm{HT}_{2 \mathrm{~B}}$ (Thamm et al., 2013). The other non-selective 5-HT receptor antagonist methiothepin interestingly had agonistic properties on the 5-HT8 receptor of the small white butterfly, Pieris rapae (Qi et al., 2014). Mianserin was shown to inhibit 5-HT2 and 5-HT7 receptors (Colas et al., 1995; Thamm et al., 2013; Vleugels et al., 2014; for an overview see Vleugels et al., 2015).

\subsubsection{Dopamine receptors}

In vertebrates, five distinct dopamine GPCRs mediate all known functions of dopamine. They can be divided in two major groups based on their structural, pharmacological and biochemical properties. The first group, called D1-class dopamine receptors (D1 and D5, which may also be referred to as D1A and D1B since they are presumably closely related paralogues that arose from a gene duplication event), couple to $\mathrm{G}_{\alpha \mathrm{s}}$ and thus increase intracellular cAMP levels. The second group, called D2-class dopamine receptors (D2, D3 and D4) mediate cAMP reduction by interaction with $\mathrm{G}_{\text {ai }}$ (Beaulieu and Gainetdinov, 2011; Callier et al., 2003; Cardinaud et al., 1998).

Because of the conservation of common functional and structural properties, the three types of dopamine receptors found in most invertebrates are generally categorised using the same classification system as vertebrates. Members of the Dop1 group are closely related to the vertebrate D1 receptor class and thus increase intracellular cAMP levels. Members of the Dop2/INDR (Invertebrate-specific Dopamine Receptors) group also show more sequence similarity with D1-like receptors than with D2-like receptors, and also increase intracellular cAMP 
levels (Blenau and Erber, 1998; Hill et al., 2016; Humphries et al., 2003; Mustard et al., 2003; Ohta et al., 2009). However, the Dop3 class is more closely related to the vertebrate D2 class, and act to reduce cAMP levels (Beggs and Mercer, 2009; Beggs et al., 2005; Beggs et al., 2011; Missale et al., 1998; Mustard et al., 2005; Verlinden et al., 2015). The Tribolium castaneum Dop3 receptor was reported to have constitutive effects on cAMP levels and upon ligand binding the receptor also increased intracellular calcium levels (Verlinden et al., 2015). An additional receptor, the Dop/Ecd, has been characterised in Drosophila melanogaster. This receptor increases intracellular cAMP levels, but does not seem to be closely related to other dopamine receptors. This receptor interacts with both dopamine and ecdysone (Evans et al., 2014; Srivastava et al., 2005).

The agonist 6-amino-5,6,7,8-tetrahydroaphthalene-2,3-diolhydrobromide (6,7-ADTN) activates Dop1, Dop2 and Dop3 receptor types (Mustard et al., 2003; Sugamori et al., 1995; Troppmann et al., 2014; Verlinden et al., 2015). Bromocriptine seems to be a potent and effective agonist for insect Dop3 receptors (Verlinden et al., 2015), with only minor or no agonist activity on Dop2 receptors (Reale et al., 1997; Troppmann et al., 2014). It may thus be a good candidate to pharmacologically distinguish D2-like receptors from D1-like receptors and INDRs in insects. A general agonist of 5-HT-receptors, 5-methoxytryptamine, also induced considerable Dop3 receptor activation (Verlinden et al., 2015).

Flupenthixol, an antagonist known to inhibit mammalian dopamine (D1-D5), serotonin (5HT2), adrenaline ( $\alpha 1$ ), and histamine (H1) receptors, is a potent antagonist of insect Dop1, Dop2 and Dop/Ecd receptors (Blenau et al., 1998; Han et al., 1996; Hearn et al., 2002; Mustard et al., 2003; 2005; Reale et al., 1997; Srivastava et al., 2005). Flupenthixol also has antagonistic properties on Dop3 receptors when applied in high concentrations (Hearn et al., 2002; Verlinden et al., 2015). The two structurally related compounds chlorpromazine and chlorprothixene antagonise the same mammalian receptors as flupenthixol and also Dop1, Dop2 and Dop3 receptors (Sugamori et al., 1995; Hill et al., 2016; Verlinden et al., 2015).

Butaclamol is a potent general dopamine receptor antagonist, inhibiting all insect dopamine receptors it was tested on. 7-chloro-8-hydroxy-3-methyl-1-phenyl-2,3,4,5-tetrahydro-1H-3benzazepine (SCH23390) seems to be a selective antagonist for Dop1 and Dop2 receptors (Hearn et al., 2002; Srivastava et al., 2005; Verlinden et al., 2015), while spiperone additionally antagonises the Dop/Ecd receptor (Srivastava et al., 2005). Mianserin and methysergide are potent antagonists for Dop3 (Verlinden et al., 2015). 


\subsubsection{Octopamine and tyramine receptors}

As indicated before, octopamine and tyramine are the invertebrate counterparts of norepinephrine and epinephrine. The vertebrate compounds interact through $\alpha$-and $\beta$-adrenergic receptors, which show considerate homology with the receptors for octopamine and tyramine.

Insect/invertebrate octopamine and tyramine receptors can be grouped into five classes: (i) $\alpha 1$-adrenergic-like octopamine receptors (Oct $\alpha 1 \mathrm{R})$; (ii) $\beta$-adrenergic-like octopamine receptors (Oct $\beta R$ ); (iii) the recently characterised $\alpha 2$-adrenergic-like octopamine receptors (Oct $\alpha 2 R$ ); (iv) octopamine/tyramine or tyramine receptors (TyrRI); and (v) tyramine receptors (TyrRII) (Evans and Maqueira, 2005; Beggs et al., 2011; Verlinden et al., 2010a; Wu et al., 2014). The Octa1R class shows sequence similarity with vertebrate $\alpha 1$-adrenergic receptors. These receptors have higher affinity for octopamine than tyramine, and induce an increase in intracellular calcium levels and also a small increase in levels of intracellular cAMP (Balfanz et al., 2005; Grohmann et al., 2003; Wu et al., 2014). The Oct $\beta R$ class has sequence similarities with vertebrate $\beta$-adrenergic receptors and specifically increases intracellular cAMP levels in response to octopamine (Hauser et al., 2006; Maqueira et al., 2005; Wu et al., 2012a). The Oct $22 \mathrm{R}$ class shows sequence similarities with vertebrate $\alpha 2$-adrenergic receptor and was only recently characterised in the rice stem borer, Chilo suppressalis. Upon activation they decrease intracellular cAMP levels and increase intracellular calcium levels. Octopamine is more potent in activating this receptor as compared to tyramine (Wu et al., 2014). The TyrRI class similarly shows structural and pharmacological similarities with vertebrate $\alpha 2$-adrenergic receptors. In most studies, these receptors have a preference for tyramine over octopamine in inducing a decrease in intracellular cAMP levels (Blenau et al., 2000; Ohta et al., 2003; Poels et al., 2001; Rotte et al., 2009; Saudou et al., 1990; Vanden Broeck et al., 1995; Wu et al., 2013), whereas in some preparations of the D. melanogaster receptor octopamine has shown to be equally or more potent as tyramine in increasing intracellular calcium release (Reale et al., 1997; Robb et al., 1994). The TyrRII class is specific for tyramine and leads to an increase in intracellular calcium levels (Bayliss et al., 2013; Cazzamali et al., 2005; Huang et al., 2009; Reim et al., 2017; Wu et al., 2015).

Naphazoline and clonidine are very potent agonists of Oct $\beta R$ and Oct $\alpha 2 R$ (Chen et al., 2010a; Maqueira et al., 2005; Wu et al., 2012a, 2014). Naphazoline also activates TyrRI and 
TyrRII receptors (Huang et al., 2009; Vanden Broeck et al., 1995). Phentolamine is capable of activating many octopamine and tyramine receptors. However, since it does not activate the receptors equally efficiently (partial agonist) and it competes over the same binding place; its presence reduces activation of the receptor by other agonists (Wu et al., 2014). Demethylchlordimeform activates the Octa1R and Oct $\beta \mathrm{R}$ of Bombyx mori (Ohtani et al., 2006; Chen et al., 2010a). Chlordimeform does not activate the TyrRI or TyrRII of Chilo suppressalis (Wu et al., 2013; Wu et al., 2015) and only induces small activity in the Octa1R of Bombyx (Ohtani et al., 2006); in high doses it has been reported to even have antagonistic effects (Nathanson and Hunnicutt, 1981).

Epinastine is a strong antagonist of Octa2R (Wu et al., 2014) and a weak antagonist of Octa1R (Huang et al., 2010), with no effect on Oct $\beta R$ (Wu et al., 2012a). Chlorpromazine and mianserin are potent antagonists of Oct $\beta R$, with no effect on Octa2R (Wu et al., 2014). Cyproheptadine and metoclopramide are antagonists of Oct $\alpha 1 R$, Oct $\beta R$ and TyrRI. Yohimbine is an antagonist of TyrRI and TyrRII (Saudou et al., 1990; Rotte et al., 2009; Wu et al., 2013), which also inhibits Octa1R (Bischof and Enan, 2004; Ohtani et al., 2006; for an overview see Verlinden et al., 2010a).

\subsection{INVOLVEMENT OF BIOGENIC AMINES AND THEIR RECEPTORS IN LOCUST PHASE TRANSITION}

\subsubsection{Serotonin}

In S. gregaria, gregarising stimuli lead to a rapid ninefold transient increase in serotonin content in the thoracic ganglia and a slower fourfold increase in both the brain and optic lobes over a $24 \mathrm{~h}$ period (Rogers et al., 2004). Later, pharmacological manipulations provided evidence that serotonin is both necessary and sufficient to initiate gregarisation (Anstey et al., 2009). Injections of serotonin, or serotonin receptor agonists (a mix of $\alpha-\mathrm{Me}-\mathrm{HT}$ and 5-CT) induced some degree of behavioural gregarisation in isolated locusts. Injections of the serotonin precursor 5hydroxytryptophan had little effect on their own, but greatly accelerated the rate of behavioural change after brief exposure to gregarising stimuli compared to controls. Conversely, $\alpha$ methyltryptophan (AMTP), an inhibitor of tyrosine hydroxylase, or a mix of serotonin receptor antagonists (ketanserin and methiothepin) prevented behavioural gregarisation in briefly crowded 
locusts (Anstey et al., 2009). More recently, immunofluorescence staining has identified serotonergic neurons in $S$. gregaria nymphs that respond to gregarising stimuli (Rogers and Ott, 2015) and has qualitatively supported the earlier finding (Rogers et al., 2004) that solitarious phase nymph ganglia contain 20-35\% more serotonin than those of gregarious nymphs. A cAMPdependent protein kinase (PKA), an intracellular effector molecule closely linked to learning and other forms of neuronal plasticity, is also required for behavioural gregarisation (Ott et al., 2012). PKA is a downstream effector of many biogenic amine receptors, including $5 \mathrm{HT}_{7}$, a likely target of serotonergic signalling during gregarisation in $S$. gregaria. Despite these several lines of evidence, the role of serotonin in behavioural phase change in S. gregaria has been challenged. Tanaka and Nishide (2013) found that injections of serotonin into the thoracic haemocoel failed to induce a gregarious-like attraction to a stimulus group, although it may be that serotonin injections are only effective with direct application onto the thoracic ganglia (Anstey et al., 2009; Rogers et al., 2014). A further analysis of gregarisation in S. gregaria, which looked at the individual behaviours used to construct the logistic regression model, suggested that behaviours related to both activity and attraction to other locusts were indeed affected by serotonin (Rogers et al., 2014; see also Section 3).

Solitarisation in $S$. gregaria has been less thoroughly analysed at the behavioural level than gregarisation, and there have been few studies looking for possible neurochemical mechanisms. The most thorough behavioural analysis suggests that after a period of rapid behavioural change in the first few hours of isolation, locusts remain in a state intermediate between gregarious and solitarious values for a period of at least $96 \mathrm{~h}$ (Roessingh and Simpson, 1994). It is unclear whether the initial rapid loss of activity following isolation is genuine solitarisation or represents a loss of arousal following the removal of stimulation from other locusts.

More recently, Alessi and co-workers (2014) analysed changes in titres of biogenic amines following isolation of long-term gregarious $S$. gregaria. Their behavioural analysis suggested that there was an exceptionally rapid loss of gregarious behaviour starting with a change in attraction / repulsion to other locusts within $1 \mathrm{~h}$ of isolation, followed by greatly decreased activity within the next $24 \mathrm{~h}$. The arena design and behavioural assay differed in some regards from the Roessinghtype design used by many other researchers (see Section 3), and there was no long-term (multigenerational) isolated solitarious group used to bench-mark fully solitarious behaviour, so its correspondence to the earlier study (Roessingh and Simpson, 1994) is unclear. Alessi et al. (2014) 
found that the amount of serotonin in the thoracic ganglia increased following as little as $1 \mathrm{~h}$ of isolation and was elevated for at least $72 \mathrm{~h}$ post-isolation, consistent with the earlier finding by Rogers et al. (2004). They also found however, that the amount of serotonin in the brain decreased significantly in contrast to the earlier study, which found a substantial increase, particularly in the optic lobes. It is clear that further work is required to unravel this complex issue.

In contrast to $S$. gregaria, levels of serotonin in the brains of L. migratoria did not differ between long-term solitarious and gregarious $4^{\text {th }}$ instar nymphs (Ma et al., 2011). Crowding solitarious nymphs for up to $32 \mathrm{~h}$ did not induce any change in brain serotonin titres (Guo et al., 2013), but it should be noted that time sequences of behavioural change in L. migratoria following crowding of isolated-reared nymphs from the same lab (Guo et al., 2011; Ma et al., 2011, 2015) suggested that gregarisation is an extremely slow process in this species, and locusts were only partially gregarised even after $64 \mathrm{~h}$ of crowding (median $P_{\text {greg }}$ 0.2-0.3 compared to median values $>0.8$ for fully behaviourally gregarious locusts, and $<0.2$ for fully solitarious locusts from the model populations). There is some evidence for serotonin's role as initiator of gregarisation in $L$. migratoria from injections into the thoracic haemocoel with serotonin receptor agonists and antagonists (Ma et al., 2011). Injections of the 5HT2 receptor agonist ( \pm )-1-(2,5-dimethoxy-4iodophenyl)-2-aminopropane hydrochloride $[( \pm)$ DOI] caused an acute shift towards gregarious behaviour when injected into the head capsule of solitarious locusts, but by contrast, locusts treated with the same agonist whilst crowded for $32 \mathrm{~h}$ showed somewhat slower behavioural change than saline injected controls (Guo et al., 2013).

Isolation of gregarious nymphs led to a progressive increase in serotonin titres in the brain until by $32 \mathrm{~h}$ the amounts were 3 -fold greater (Guo et al., 2013). Here again, the correlation with the change in behaviour was not particularly strong: L. migratoria appears to undergo a very rapid behavioural solitarisation, with gregarious locusts isolated for just one hour showing a substantial loss of gregarious behaviour, and locusts isolated for $4 \mathrm{~h}$ or longer indistinguishable from longterm solitarious locusts (Guo et al., 2011). Serotonin had no acute effect on the behavioural phase state of gregarious locusts when injected, but there was evidence that it acted to accelerate the rate of solitarisation when combined with brief periods of isolation (Guo et al., 2013). The long-lasting increase in serotonin on isolation, well beyond the full expression of fully solitarious behaviour, suggests that it may have a role in the further consolidation of phase state. It is currently unknown how long the elevated serotonin titre lasts and what its full functional significance is. 
This suggests that serotonergic signalling in L. migratoria is linked to both behavioural solitarisation and gregarisation. There are several potential explanations for the seemingly contradictory effects of serotonin on behavioural phase change in L. migratoria. First, specific serotonin receptor classes could carry out different functions in solitarisation or gregarisation. This is supported by the finding that mRNA expression of only the $5 \mathrm{HT}_{2}$ receptor increased during behavioural gregarisation, and injecting a specific $5 \mathrm{HT}_{2}$-receptor agonist directly into the brain of solitarious nymphs induced gregarisation (Guo et al., 2013). However, RNAi against the 5HT2 receptor did not influence phase state or behavioural gregarisation relative to controls (Guo et al., 2013). Second, there could be time- and tissue-specific effects of serotonin on behavioural phase change, as brain serotonin levels in L. migratoria change in response to solitarisation but systemic injections primarily affected gregarisation (Ma et al., 2011). It is possible that the role of serotonin is to facilitate behavioural/neuronal plasticity during both gregarisation and solitarisation, but does not have any direct effect on the expression of gregarious or solitarious behaviours themselves. Full phase change is accompanied by extensive changes in gene expression (Kang et al., 2004; Badisco et al., 2011a,b; Guo et al., 2011), which means that signalling systems are acting on different underlying physiologies in the two phases and may therefore have different effects. Further work is required to characterise potentially distinct neuromodulatory roles of serotonin in different regions of the nervous system.

\subsubsection{Dopamine}

Dopamine plays important roles in social interactions in a range of insects (Penick et al., 2014; Rillich and Stevenson, 2014; Vergoz et al., 2009), so it is plausible that it would also play a role in behavioural phase change. Crowding solitarious S. gregaria nymphs transiently increased dopamine content in the brain and thoracic ganglia, but the time course was slower than that of behavioural gregarisation (Rogers et al., 2004). Upon isolating S. gregaria, brain dopamine titres also increased transiently (Rogers et al., 2004; Alessi et al., 2014), suggesting that there may be a role for dopamine in the solitarisation process. Alessi et al. (2014) found an increase within one hour of isolation which was maintained for at least $72 \mathrm{~h}$, while Rogers et al. (2004) only examined a single $24 \mathrm{~h}$ isolation time point. Injecting gregarious and solitarious $S$. gregaria with dopamine increased the likelihood of test locusts avoiding other locusts in a behavioural assay whereas 
fluphenazine, an antagonist of vertebrate D1 - and D2-class receptors, increased their propensity to approach other locusts (Alessi et al., 2014).

Ma and co-workers (2011) studied the catecholamine pathway in L. migratoria and reported lower amounts of dopamine in solitarious nymphs compared with gregarious nymphs. Brain dopamine titres in solitarious nymphs transiently increased above long-term gregarious values upon crowding, which corresponded with an upregulation of the Dop1 receptor (Guo et al., 2015) and an increased expression of genes in the dopamine synthesis pathway (Ma et al., 2011), (but see comments on the time course of behavioural change in L. migratoria above). Gregarious locusts displayed more solitarious-like behaviour when i) dopamine biosynthesis was interfered with using the tyrosine hydroxylase inhibitor AMTP, ii) when dopamine release was prevented by reserpine, an irreversible blocker of the vesicular monoamine transporter (which affects all monoamines) iii) when dopamine receptor signalling was inhibited by SCH23390, an antagonist of Dop1 and Dop2 receptors, (Ma et al., 2011), and iv) following RNAi-mediated knockdown of Dop1 (Guo et al., 2015). SKF38393, an agonist of vertebrate D1-class receptors, induced an acute change towards gregarious behaviour in solitarious nymphs, and also accelerated the shift towards gregarious behaviour upon crowding, and was reported to affect both activity and attraction towards conspecifics (Guo et al., 2015). Dopamine acting via Dop1 thus seems to play a role in gregarisation of L. migratoria.

In gregarious L. migratoria nymphs, there was a drop in dopamine titre in the brain after only $1 \mathrm{~h}$ of isolation, which mirrored an upregulation of the Dop2 receptor (Guo et al., 2015). Solitarious nymphs injected with a vertebrate D2-class receptor agonist R(-)-2,10,11-trihydroxyN-propylnoraporphine hydrobromide hydrate [R(-)-TNPA], and left uncrowded, did not display any behavioural change. Gregarious locusts treated with the Dop2 antagonist S(-)-sulpiride and subsequently isolated were much more gregarious in behaviour after $1 \mathrm{~h}$ than control animals (i.e. the rate of solitarisation had been slowed down). A similar result was seen when RNAi was performed against the Dop2 receptor (Guo et al., 2015). Dop2 signalling could thus be needed for solitarisation in both L. migratoria and S. gregaria.

\subsubsection{Octopamine and tyramine}


There has been relatively little attention to the role of octopamine and its precursor tyramine in the behavioural phase change of $S$. gregaria. Tyramine titres across the whole nervous system decreased upon isolating gregarious nymphs, but there was no noticeable change in octopamine (Rogers et al., 2004). Conversely, in a separate study of gregarious adults, isolation corresponded with a fall in octopamine titres in the metathoracic ganglia after $1 \mathrm{~h}$ of isolation and levels stayed low until $24 \mathrm{~h}$ of isolation, but there was no change in tyramine levels (Alessi et al., 2014). In $S$. gregaria, Octa1R and Oct $\beta \mathrm{R}$ transcript levels are significantly higher in long-term gregarious animals as compared to long-term solitarious animals. The transcript levels of Oct $\beta R$ in the central nervous system are already significantly increased after $30 \mathrm{~min}$ of crowding (Verlinden et al., 2010b).

In L. migratoria, early studies reported elevated levels of octopamine in the brains of solitarious animals (Fuzeau-Braesch and David, 1978; Fuzeau-Braesch and Nicolas, 1981). Ma et al. (2015) made a weakly supported claim for lower titres of octopamine in solitarious nymphs compared to long-term gregarious nymphs (see their Fig. 2A; four of the six measurements for gregarious locusts are similar to those of solitarious locusts). In the same study, isolation of gregarious nymphs was accompanied by an increase in the amount of tyramine according to the duration of isolation. Locusts in these experiments were placed in a Y-maze and given the choice of odour volatiles from a group of gregarious locusts or a clean air supply following crowding or isolation treatments. In gregarious locusts that had been isolated the amount of tyramine in the brain was positively correlated with the percentage of locusts choosing the clean-air arm of the $\mathrm{Y}$ maze (Ma et al., 2015; see Fig. 2C therein). There was no clear trend in the expression of a tyramine receptor during isolation. The involvement of tyramine signalling in the early stage of solitarisation was supported by a dramatic shift towards solitarious-like behaviour in gregarious locusts given systemic injections of tyramine, and RNAi against TyrR1 which, whilst not affecting gregarious behaviour, appeared to protect against the loss of gregarious behaviour when locusts were isolated.

Expression of the octopamine receptor Octa1R showed no change during isolation. Ma et al. (2015) also reported that the decrease in brain octopamine levels during isolation corresponded with a loss of attraction to locust odours. However, this interpretation is only possible because of two extreme outliers in the long-term gregarious group in the data (see their Supplementary Fig. $4 \mathrm{~A}$, in which the data points for gregarious and solitarious octopamine titres are duplicated from Fig. 2A in the main text). 
Upon crowding, tyramine- 3 -hydroxylase, the enzyme which converts tyramine into octopamine, may be upregulated. There was also evidence for an increase in the expression levels of the Oct $\alpha 1 \mathrm{R}$ during crowding, correlated with the increased attraction to conspecifics, but expression in long-term gregarious and solitarious locusts appears more similar. There was no correlation between total brain octopamine concentration and attraction to locust volatiles in a Ymaze. Systemic injections of octopamine into solitarious locusts promoted a small increase in gregarious-like behaviours and some alteration in odour preferences in a Y-maze towards preference for conspecific odours. RNAi against the Octa1R had no effect on solitarious behaviour in a behavioural assay, but treated locusts failed to show any behavioural change following $32 \mathrm{~h}$ of crowding, unlike saline treated controls (Ma et al., 2015). There may therefore be a role for octopamine in L. migratoria during gregarisation and in mediating the attraction to locust odour in solitarious nymphs during crowding.

\subsubsection{Conclusions}

Given the complex ways that biogenic amines act on a wide range of behaviours and processes (Andretic et al., 2005; Buhl et al., 2008; Goosey and Candy., 1908; Leitch et al., 2003), it is challenging to identify key mechanisms that control phase change, as these amines are not uniquely linked to single functions, interact with a range of receptors, and likely have a mix of mutually synergistic and antagonistic relationships with each other in modulating plasticity and behaviour. Many studies have utilised pharmacological or molecular interventions that may be expected to have very wide-ranging and systemic effects beyond behavioural phase change. A major challenge is to identify and zoom in on the specific neural circuits and systems mediating behavioural phase change. Very strong effects have been reported for different individual biogenic amine and receptor combinations on both gregarisation and solitarisation within the same species. There is a need to improve our understanding of how these separate elements work together to produce the phenotypic change characteristic of natural behavioural phase change. There is considerable variation in the time course of phase change both within and across locust species, further stressing the importance of taking a holistic and multidisciplinary approach to uncovering the underlying mechanisms. 


\section{Genomics, transcriptomics and epigenetics}

Transcriptomic studies in a range of insect species have shown that differential gene expression is an important mechanism underlying phenotypic plasticity, for example in the determination of caste in eusocial insects (Oppenheim et al., 2015; Corona et al., 2016). Since locusts can produce two dramatically different phenotypes from a single genome, some gene products must either be differentially expressed, or have differential activity or function between phases. Most quantitative transcriptomic studies in locusts have focussed on differences between long-term phases (in $L$. migratoria and S. gregaria). Many of the earlier studies (Kang et al., 2004; Wei et al., 2009; Chen et al., 2010b) lacked substantive biological replication, and although different transcript families and life stages have been targeted, our understanding of the transcriptomic underpinnings of phase polyphenism is still fragmentary.

\subsection{AVAILABILITY OF SEQUENCE INFORMATION}

The availability of sequence information has long been a limiting factor in the quest for key molecular regulators of locust phase change, but substantial progress has been made in recent years. The first large resources of orthopteran transcriptome sequences were databases of Sangersequenced Expressed Sequence Tag (EST) clones for L. migratoria (Kang et al., 2004; Ma et al., 2006) and S. gregaria (Badisco et al., 2011a). Although contig numbers were similar for both species (approximately 12,000) there was only limited sequence overlap between both sets, which may be explained by the use of RNA extracts from different tissues or life stages and/or by EST clones not typically covering the entire length of mRNA transcripts. These databases also provided annotation information using classifications frameworks such as Clusters of Orthologous Groups (COG), Gene Ontology (GO), the Kyoto Encyclopedia of Genes and Genomes (KEGG) and InterPro, but about half the sequences remain unannotated. Microarrays have also been designed for L. migratoria and S. gregaria to study various aspects of phase polyphenism (e.g. Badisco et al., 2011b; Ma et al., 2011; Guo et al., 2011).

With the advent of next-generation sequencing (NGS) technologies, especially Illumina sequencing, the number of available insect transcriptomes is growing rapidly, with the ever- 
broadening range of taxa including several orthopteran species (Oppenheim et al., 2015). To date, the most extensively covered orthopteran species is L. migratoria, with transcriptome projects based on different tissues, life stages and transcript families (Wei et al., 2009; Chen et al., 2010b; Ma et al., 2011; Wei et al., 2011), and a recently-published reference genome with 17,307 predicted genes (Wang et al., 2014), the first published genome for any locust species.

\subsection{TRANSCRIPTOMIC DIFFERENCES BETWEEN LONG-TERM PHASES}

The first quantitative transcriptomic study in L. migratoria compared the transcriptomes of solitarious and gregarious 5th instar nymphs, using cDNA derived from either the head, hind legs or midgut (Kang et al., 2004). A remarkable observation was the higher expression of juvenile hormone binding proteins (JHPH superfamily of proteins: juvenile hormone binding protein, hexamerins, prophenoloxidase and hemocyanins) in the heads of gregarious nymphs. More general patterns of tissue-specific differential expression were also observed, and the authors claimed a total of 532 transcripts as being putative differentially-expressed genes (DEGs). However, since this study lacked biological replication, it is hard to estimate the real extent of differential gene expression.

The first publication to use NGS in a study of locusts (Wei et al., 2009) focussed on the small RNA fraction of the transcriptome of L. migratoria. Small RNAs belonging to 50 known gene families were found, and 185 new, potentially locust-specific microRNA (miRNA) transcripts were predicted. This study claimed substantial differences in miRNA expression between long term phases. However, these claims were based on the comparison of two samples that seemed to differ substantially in their content of tRNA, rRNA and unannotated small RNA, suggesting that technical differences between both cDNA libraries may have existed that could equally well explain the differences in miRNA. Shortly after this, Chen et al. (2010b) published an updated L. migratoria transcriptome using Illumina sequencing based on whole body RNA extracts of gregarious and solitarious animals of different ages. This transcriptome contained 72,977 contigs larger than 100 base pairs, with 11,490 predicted protein-coding genes. During early larval stages, transcriptomic differences became increasingly pronounced, culminating in the most substantial difference recorded at the 4 th instar stage. In older nymphs and adults, the

differences were smaller again. Since only one replicate was used per age cohort, it was still very 
hard to quantify the extent of differential gene expression, but a core set of 242 genes differed between both phases throughout development and adult life. These transcripts were suggested to be candidate phase marker genes. Most of these genes were involved in metabolism and biosynthesis, but a number of signal transduction components were also differentially expressed. Ma et al. (2011) focussed on the differences between long term phases of L. migratoria throughout development using a microarray. They also found that differences between both phases peaked at the 4th instar stage, supporting the findings of Chen et al. (2010b) and suggesting that the 4th instar is a key developmental stage for the determination of phase state. Among the 594 Differentially Expressed Genes (DEGs) at the 4th instar stage, the most common functional categories were general metabolism, molecular transport, the production of cuticle protein, and chemosensory transduction. The molecular pathway of catecholamine biosynthesis and metabolism ranked as the most enriched pathway in genes upregulated in gregarious locusts. The important role of this pathway was further confirmed with quantitative PCR and functional experiments (see Section $5.2)$.

The first transcriptomic comparison between long term phases of $S$. gregaria was a microarray study by Badisco et al. (2011b). The brain transcriptome of adult solitarious and gregarious locusts was compared and 214 transcripts were found to be differentially expressed. In gregarious brains, DEGs were enriched in stress response genes including heat shock proteins, a group of genes that had already been shown to be differentially expressed in L. migratoria (Wang et al., 2007). Conversely, solitarious locusts seemed to invest more in oxidative stress resistance, which may facilitate their increased longevity compared to their gregarious counterparts (Maeno et al., 2013). Much as in L. migratoria, long term phases also differed in metabolism, sensory perception, signal transduction and some developmental pathways (despite the maturity of the subjects).

\subsection{THE TRANSCRIPTOME DURING PHASE TRANSITION}

The first transcriptomics study focussing on the time-course of behavioural change was a microarray study in L. migratoria by Guo et al. (2011). 794 transcripts were shown to be differentially expressed at some point during a 64-h isolation of gregarious 4th instar nymphs, and 1103 were differentially expressed at some point during a 64-h crowding of solitarious 4th instar 
nymphs. Solitarisation appears to be a relatively fast process in L. migratoria, which was reflected in large fluctuations in the patterns of differential expression. On the other hand, gregarisation seems to take much longer (see Section 5.2), which was reflected by more stable trends in differential expression. In both time-courses, insect cuticle proteins, pheromone binding, odorant binding and members of the JHPH family were most enriched among DEGs. Two groups of antenna-specific DEGs were also functionally validated; several chemosensory proteins and takeout appear to play a role in olfaction-led group attraction and repulsion by gregarious and solitarious locusts, respectively (see Section 3.2).

A transcriptomic study was also published alongside the L. migratoria reference genome (Wang et al., 2014), using a similar 64-h time-course. The authors stated that 4,893 genes were differentially expressed at some point over this time-course (i.e. $28.3 \%$ of all predicted genes in their genome), but this was based on only one replicate per time point. Furthermore, a list of these putative DEGs, or any details concerning their annotations or functions, was not given.

\subsection{MOBILE ELEMENTS}

Orthopterans have a wide range of genome sizes, and include some of the largest known insect genomes (Westerman et al., 1987; Wang et al., 2014; Camacho et al., 2015). One possible driver of genome size expansion is colonization by mobile DNA elements. Jiang et al. (2012) screened the transcriptome of L. migratoria for retrotransposons and found evidence for transcription of retroelements from approximately 100 loci in the genome, a figure comparable to observations in D. melanogaster and the malarial mosquito Anopheles gambiae. Locusts did however, show a higher proportion of non-Long Terminal Repeat (non-LTR) retroelements (73 non-LTR versus 32 LTR retroelements) with a greater diversity (members of 9 families versus 4 or 5 in dipterans) and with higher numbers of divergent copies. They also had a higher number of intact Open Reading Frames (ORFs) suggesting retrotransposition is still an active process. Based on observed differences in retroelement transcription between both phases in 5th instar and adult animals (but not in earlier life stages), the authors suggested that somatic retrotransposition may play a role in the regulation of phase-related phenotypic plasticity.

The reference genome of L. migratoria (Wang et al., 2014) estimated the haploid genome size to be 6.3 gigabases in this species. Large introns and repetitive elements (both intergenic and 
in introns) were the apparent reasons behind this large genome size, covering about $60 \%$ of the total assembly. These elements mainly consisted of DNA-transposons and long interspersed elements (LINEs). The latter was the most prevalent repeat family (about $4 \%$ of the genome) and contained many full-length copies with intact ORFs. This may be an indication of active transposition, which is in accordance with the earlier observed transcription of non-LTR retrotransposons (Jiang et al., 2012). Transposon diversity was high, with no single transposon dominating, indicating a general susceptibility to transposition rather than an aggressive colonization by a single element.

No other locust genomes have been sequenced so far. Based on Feulgen Image Analysis Densitometry and C-banding techniques, the haploid genome size of S. gregaria was estimated to be around 8.55 gigabases (Camacho et al., 2015). Though transposons were not the focus of this study, non-LTR transposons were also identified as an important part of repetitive DNA. In a microarray study comparing both phases in S. gregaria, one reverse transcriptase had a higher expression in the solitarious phase (Badisco et al., 2011b).

\subsection{DNA METHYLATION IN LOCUSTS}

Together with chromatin modifications and non-coding RNAs, cytosine methylation of DNA has emerged as a key epigenetic mechanism in regulating gene expression to tailor phenotypes to the environment (Szyf et al., 2008; Angers et al., 2010; Dias, 2015; Isles, 2015). In social insects, DNA methylation has been implicated in the differential gene expression that underpins caste polyphenism and the division of labour (Yan et al., 2015). It is therefore reasonable to expect that DNA methylation has a role in phase polyphenism and phase-related behavioural plasticity.

In mammals, DNA methylation is accomplished by two classes of DNA (cytosine-5)methyltransferases, DNMT1 and DNMT3. It is commonly assumed that DNMT3 is responsible for establishing methylation patterns de novo, thus interfacing the 'methylome' with the environment, whereas DNMT1 maintains the established pattern during cell divisions. A further enzyme, DNMT2, was so named due to its pronounced sequence similarity with DNMT1, but does not appreciably methylate DNA in mammals. Instead, DNMT2 very efficiently methylates aspartate tRNA (Goll et al., 2006) although a dual role in both tRNA and DNA methylation remains a possibility in some organisms (Jeltsch et al., 2016). 
In locusts, the evidence for the existence of the Dnmt 1 and Dnmt 2 genes is uncontroversial. Two Dnmt1 paralogues and one Dnmt2 are present in the genome of L. migratoria (Robinson et al., 2011, 2016; Wang et al., 2014). In S. gregaria, where whole-genome information is not yet available, current evidence indicates the presence of at least one Dnmtl (Falckenhayn et al., 2013) and one Dnmt2 (Boerjan et al., 2011). By contrast, the search for DNMT3 has long been unsuccessful in both species. cDNA fragments related to Dnmt 3 are absent in the published $S$. gregaria (Badisco et al., 2011a) and L. migratoria (Ma et al., 2006) EST databases (Robinson et al., 2011). Analysis of S. gregaria transcriptome-scale shotgun cDNA sequences gave 75 BLAST hits (E-values $<0.1$ ) with Apis mellifera DNMT1a as query, but no hits with A. mellifera DNMT3 (Falckenhayn et al., 2013). Subsequently, the genome announcement of Wang et al. (2014) reported the presence of a Dnmt3 gene in L. migratoria, but did not present evidence in the paper. We have verified the presence of a hypothetical DNMT3-like protein, LOCMI17543-m1, in the current version of the LocustBase Official Gene Set (OGS CDS V2.4.1) associated with Wang et al. (2014). LOCMI17543-m1 shows only weak sequence conservation with other DNMT3s (about $30 \%$ over a query cover of approximately $95 \%$ in tblastn searches against the NCBI $n t$ nucleotide collection database), but all top-scoring hits are Dnmt $3 b$ representatives and the three canonical domains present in mammalian DNMT3b (PWWP, ADDz_Dnmt3 and Dcm) can be identified by Conserved Domain Database BLAST (Ott, unpublished). Interestingly, however, BLASTsearching a L. migratoria CNS transcriptome (41,179 sequences; Zhang et al., 2012) with LOCMI17543-m1 as query gave no hit (Ott, unpublished). Robinson et al. (2016) likewise reported little evidence for DNMT3 expression in publicly available L. migratoria transcriptome datasets.

Possible explanations for the elusiveness of Dnmt3 transcripts in locust EST and transcriptome data are either first, that expression is much lower than for Dnmt1 and Dnmt2, or second, that the Dnmt3-like sequence in the L. migratoria genome (Wang et al., 2014) is no longer a functional gene. Locusts would not be alone among insects with functionally important DNA methylation to lack DNMT3 (Bewick et al., 2016). In these insect species, its function is possibly taken over by DNMT1.

There is some evidence that DNMTs are differentially expressed between phases. In $L$. migratoria, Dnmt1 is more strongly expressed in the eggs and ovaries of solitarious locusts, and Dnmt2 in nervous tissue of solitarious males (Benjamini-Hochberg corrected, $\alpha=0.05$; Robinson 
et al., 2016). In S. gregaria, Boerjan et al. (2011) reported differential expression of Dnmt1 and Dnmt2 in several of the 15 different tissues investigated, including in brain, testes and muscle (based on $N=3$ batches of pooled tissues per phase, not corrected for multiple comparisons).

Irrespective of the uncertainty surrounding the presence of DNMT3, locust genomes are extensively methylated by insect standards. Boerjan et al. (2011) quantified the ratio of methylated to total cytosines $(\mathrm{mC} / \mathrm{C})$ in CNS-derived DNA in $S$. gregaria by mass spectrometry and reported values of $1.6 \%$ and $1.9 \%$ in the metathoracic ganglion and brain, respectively. Comparable values were subsequently obtained by genome-scale bisulphite sequencing (Falckenhayn et al., 2013), which also revealed that $>90 \%$ of $\mathrm{mC}$ residues occurred in a $\mathrm{CpG}$ dinucleotide context, as expected from other animals. These $\mathrm{mC} / \mathrm{C}$ ratios are over ten times higher than in honeybees $(0.11 \%$; Lyko et al., 2010). Because methylcytosine shows hypermutability to thymidine, the ratio of observed to expected $\mathrm{CpG}$ sites $(\mathrm{CpG} / \mathrm{E})$ allows inference about the extent of historic DNA methylation in the germline. Assessing $\mathrm{CpG} / \mathrm{E}$ across many genes yields unambiguously bimodal distributions in both L. migratoria (Robinson et al., 2011; Wang et al., 2014) and S. gregaria (Falckenhayn et al., 2013), with one mode close to 1.0 and a second mode below 0.5 , the latter indicating a large population of genes with a germline history of extensive methylation. In L. migratoria this bimodal pattern was also observed in a subset of genes that have previously been found to be differentially expressed between phases (Robinson et al., 2011). Genes with historical germ-line hypermethylation (low $\mathrm{CpG}_{O / E}$ ) were significantly associated with the GO terms metabolism, protein transport, intracellular protein transport, biosynthesis and electron transport. However, genes with a $\mathrm{CpGo/E}$ close to 1.0 may still undergo phase-related changes in methylation during the lifetime of a locust. If, for example, behavioural phase change were underpinned by hypermethylation of particular genes specifically in the CNS, this would leave no trace in the germ line.

Genome-wide analyses of methylation patterns with single-gene resolution have proved difficult in locusts due to the large size of their genomes (Wang et al., 2014; Camacho et al., 2015). Using shotgun bisulphite sequencing of genomic DNA from the CNS of gregarious S. gregaria, Falckenhayn et al. (2013) obtained 180 million sequence reads corresponding to $32 \mathrm{~Gb}$ of DNA sequence. However, the absence of a reference genome precluded the assembling into a genomewide methylation map, and the absence of data from solitarious phase DNA precluded the identification of phase differences. Using the S. gregaria EST database of Badisco et al. (2011a) 
as a reference, the $\mathrm{mC} / \mathrm{C}$ ratio in genic sequences was found to be over $3 \%$, markedly higher than the genomic ratio. This suggested that methylation might preferentially target exons, as had been previously found in other insects. Contig methylation densities showed an extremely bimodal distribution, with about $20 \%$ of contigs fully methylated ( $\mathrm{mCpG} / \mathrm{CpG}>0.95)$ and around $45 \%$ entirely unmethylated. This large proportion of densely methylated exons was in marked contrast with previously analysed insect species, but may not be unique to locusts (Bewick et al., 2016).

Wang et al. (2014) analysed the methylation of brain DNA in solitarious and gregarious fourth-instar females of L. migratoria by reduced representation bisulphite sequencing. The overall genomic $\mathrm{mC} / \mathrm{C}$ ratio was $1.6 \%$, comparable with that found in S. gregaria. This figure rose to $11 \%$ for $\mathrm{CpG}$ sites across the genome, and to $25.5 \%$ for gene-body CpGs. Strikingly, however, the exonic $\mathrm{mCpG} / \mathrm{CpG}$ ratio was only $12.8 \%$, less than $2 \mathrm{x}$ the intergenic ratio of $7.3 \%$ and only marginally higher than the genomic ratio of $11 \%$. The high gene-body $\mathrm{mCpG} / \mathrm{CpG}$ ratio of $25.5 \%$ instead reflected an intronic ratio of 31\% (all values measured from Fig. 2B of Wang et al., 2014). The intronic ratio is thus almost $2.5 \mathrm{x}$ higher than the exonic ratio. This contrasts sharply with honeybees where the $\mathrm{mCpG} / \mathrm{CpG}$ ratio is about 40x higher in exonic than in intronic DNA (Lyko et al., 2010). The ratios reported in L. migratoria also contrast with the available evidence in $S$. gregaria, where methylation appears to preferentially targets exons, with $40 \%$ of exonic sequences showing methylation ratios greater than $65 \%$ (Falkenhayn et al., 2013). The available data in the two species are not directly comparable, however, and a direct characterisation of exonic and intronic methylation densities in $S$. gregaria is needed to resolve whether locust species differ fundamentally in this respect. In both L. migratoria and S. gregaria, methylation occurs in a significant fraction of repetitive DNA, including transposons and rDNA repeats (Robinson et al., 2011, 2016; Falckenhayn et al., 2013). In L. migratoria, the average $\mathrm{mCpG/CpG} \mathrm{ratio} \mathrm{in} \mathrm{DNA}$ repeats is $15.5 \%$ (Wang et al., 2014). The supplementary material of Wang et al. (2014) lists 90 genes that showed differential methylation in the brains of solitarious and gregarious nymphs. Many of these genes were also differentially expressed according to a 'false discovery rate of the significant level of the differential expression'. A sizeable proportion of the differentially methylated genes were functionally classified as coding for kinases, other signal transduction proteins, and proteins implicated in microtubule cytoskeleton organization. This implies long-term differences in neuronal signalling and microtubule dynamics between phases, an interesting 
finding that must, however, not be confused with evidence for a role of these genes in the process of phase change.

In an alternative approach, Mallon et al. (2016) used methylation-sensitive amplified fragment length polymorphisms (MS-AFLP) to compare DNA methylation patterns in the CNS (brain and thoracic ganglia) of long-term solitarious, long-term gregarious and recently crowded (24 h) solitarious adults in S. gregaria. MS-AFLP is a gel-based fingerprinting technique that can provide an index of 'epigenetic differentiation' $\left(\Phi_{S T}\right)$ similar to the $F_{\mathrm{ST}}$ index of genetic differentiation in population genetics. A major limitation of MS-AFLP is that it screens only a limited number of anonymous loci. The methylation fingerprints of solitarious and gregarious $S$. gregaria showed significant differentiation across 282 methylation-susceptible loci. However, crowding for $24 \mathrm{~h}$ resulted in a methylation fingerprint that was differentiated from both solitarious and long-term gregarious locusts. Furthermore, in a principal coordinate analysis, the fingerprints of these recently gregarised locusts occupied a position distinctly away from the axis defined by the two long-term phases. This suggests that methylation patterns seen after $24 \mathrm{~h}$ of crowding are not simply partial realisations of the endpoints, but reflect a distinct transitional epigenetic state. This finding adds to previous evidence for the existence of mechanistically distinct transitional phase states at different levels from the molecular to the behavioural (Rogers et al., 2004; Simões et al., 2013; Rogers and Ott, 2015).

In summary, locusts have more densely methylated genomes than most other insects analysed to date (Bewick et al., 2016). One might be tempted to speculate whether this directly reflects their capacity for pronounced phenotypic plasticity, but this simplistic idea is easily refuted: the Meadow Grasshopper, Chorthippus parallelus is incapable of phase change and has a genomic $\mathrm{mC} / \mathrm{C}$ ratio of $4 \%$ (Lechner et al., 2013); and the German Cockroach, Blattella germanica has genomic and exonic $\mathrm{mCpG} / \mathrm{CpG}$ ratios of $12 \%$ and $40 \%$, respectively (Bewick et al., 2016). Similarly, the notion that DNA methylation in insects is required for caste polyphenism and the evolution of sociality is not supported by recent analyses (Standage et al., 2016; Bewick et al., 2016). Diptera show no appreciable DNA methylation, demonstrating furthermore that the extreme developmental plasticity manifest in holometabolous insects can be achieved entirely through other regulatory mechanisms. At this point, there is clear evidence for phase-related differences in DNA methylation, but we are a long way from understanding the mechanistic role of DNA methylation in phase change. The results of Mallon et al. (2016) indicate that further progress will require 
analyses of changes in DNA methylation across different time points during phase transitions, rather than just comparisons of endpoints.

\section{$7 \quad$ Neurophysiological consequences of phase change}

Although a great deal of effort has been focussed on the mechanisms and plasticity underlying phase transition, analyses of the endpoints of this process - long term solitarious and gregarious animals - have also revealed important insights through comparative analyses of how altered neuronal function relates to changes in behaviour. Phase transition requires extensive changes in the ways in which sensory information is gathered, processed and used to shape behaviour. Understanding these differences at the cellular and circuit levels will permit a deeper understanding of how processes ranging from short-term modification of existing neural structures through to the epigenetic regulation of developmental gene expression give rise to markedly different behavioural phenotypes.

Blackburn et al. (2010) related differences in the walking behaviour of adult solitarious and gregarious $S$. gregaria to the activity of one of the motor neurons controlling hind leg movements. Solitarious locusts generally walk slowly with the body held low to the ground as part of their behaviourally cryptic phenotype, but gregarious locusts walk rapidly with the body held high. Gregarious locusts take larger steps than solitarious locusts, with the femoro-tibial joint undergoing larger excursions and being kept more extended throughout the entire step cycle. The metathoracic slow extensor tibiae (SETi) motor neurone of solitarious locusts fired tonically at 514 spikes s $^{-1}$ in quiescent animals. By contrast, the SETi of gregarious locusts showed little tonic activity (0-3 spikes $\left.\mathrm{s}^{-1}\right)$. Imposed flexion of the femoro-tibial joint elicited strong transient spiking activity in the SETi of both phases, resisting the imposed movement. The spiking rate of SETi in solitarious locusts during these resistance reflexes was twice as great as that of gregarious locusts regardless of femoro-tibial joint angle or excursion, indicating a higher gain of the sensory-motor feedback loop from the main femoro-tibial joint proprioceptor. The extensor tibiae muscle of solitarious locusts produces less force than that of gregarious locusts at low stimulation frequencies (Blackburn, 2005), but the high gain of the sensory-motor feedback loop allows for fine-grained control of muscle force amplitude and thus movements (Cruse and Schmitz, 1983; Cruse, 1985). 
This in turn may be linked to the occurrence of pronounced behavioural catalepsis (Rogers and Simpson, 2014) in solitarious locusts, similar to that seen in other cryptic insects such as phasmids.

\subsection{SOCIAL COMPLEXITY AND BRAIN SIZE}

The social interactions shown by gregarious locusts lack the behavioural complexity of eusocial Hymenoptera and termites. Nevertheless, the organised collective motion of locust swarms is, like many complex social behaviours, maintained by positive and negative behavioural feedback loops arising entirely from local interactions between near neighbours, which elicit simple behavioural responses that constitute rules of interaction (Buhl et al., 2006, 2011, 2012; Rogers and Buhl, 2016). In C. terminifera, for example, alignment of walking individuals only occurs if locusts are closer than $13.5 \mathrm{~cm}$ (Buhl et al., 2011). Above a critical population density, these local interactions on the scale of a few centimetres can generate highly ordered marching behaviour within groups of locusts that extend for hundreds of metres (Buhl et al., 2006). This does not require sophisticated large-scale coordination, but comes about solely as a result of local self-organisation (Camazine et al., 2003).

The inherent sensory complexity of a locust's immediate environment greatly increases when locusts become gregarious and form dense, constantly moving groups that demand dynamic responses. Gregarisation requires that locusts adopt new behaviours, and subjects them to different kinds of risk. This includes intense competition for resources such as food and roosting sites and, at its most extreme, this competition can result in an increased threat of cannibalism (Bazazi et al., 2008, 2011; Hansen et al. 2011).

The demands that group living places on behaviour and the nervous system may be indicated by the $30 \%$ larger brain of gregarious compared to solitarious $S$. gregaria, despite gregarious locusts being typically smaller in body mass (Ott and Rogers, 2010). This increased volume is not uniformly distributed: neuropiles associated with higher-order processing and integration of sensory information are disproportionately affected. For example, gregarious locusts have smaller eyes than solitarious locusts (Dirsh, 1953; Rogers et al., 2010) and the first visual neuropile in the optic lobes, the lamina, is little different between phases. However, the second visual neuropile, the medulla, is disproportionately larger in gregarious locusts than in solitarious locusts and the third visual neuropile, the lobula, is disproportionately larger again. Likewise, in 
the olfactory system, gregarious locusts have fewer olfactory sensilla than solitarious locusts ( $L$. migratoria: Greenwood and Chapman, 1984; S. gregaria: Ochieng' et al., 1998). The antennal lobe, which is the primary olfactory neuropile, is $10 \%$ smaller in gregarious locusts than in solitarious locusts of matching brain size. The primary calyx of the mushroom bodies, a higherorder olfactory and multi-modal integration centre, is conversely 50\% larger in gregarious compared to solitarious locusts. Phase-related differences in brain region proportions seem to arise from two processes: first, allometric growth as overall brain size increases; and second, a mosaic of step changes in size of some neuropiles, for example, the central complex. These differences in brain region size were found between adult long-term (several generation) solitarious and gregarious locusts. It is unclear at present whether regional size differences are the result of neuronal proliferation or arise from the growth of existing arborisations. It is also unknown how phase change initiated during post-embryonic development affects the subsequent growth and development of the brain as the locust adopts new life history strategies.

\subsection{LEARNING ABILITIES OF SOLITARIOUS AND GREGARIOUS LOCUSTS}

Locusts, in common with many insect species, have a considerable capacity for learning (Simões et al., 2011, 2012, 2013, 2016), which is one of the most basic and universal manifestations of phenotypic plasticity. Although the ability to learn confers fitness advantages (Dukas, 2008a,b), it

can also incur substantial costs (Mery and Kawecki, 2004; 2005). Consequently, the learning abilities of different species are often fine-tuned to their specific ecological and life-history requirements and show wide variation in performance (Dukas, 2008a; Ngumbi et al., 2012). It is therefore perhaps unsurprising that solitarious and gregarious $S$. gregaria, with their very different life-histories, also differ in their learning abilities as shown in a series of papers by Simões and colleagues $(2011,2012,2013$, and 2016).

\subsubsection{Appetitive and aversive olfactory learning in gregarious locusts}

Simões et al. (2011) first developed a paradigm using restrained S. gregaria that demonstrated classical Pavlovian associative conditioning for appetitive stimuli (Simões et al., 2011). This assay was based on the palp opening reflex (POR) in which locusts that are presented with a food 
stimulus (in this case a synthetic food) extend their maxillary and labial palps. This simple reflex can be used to measure memory acquisition. Locusts can learn to associate a food stimulus (unconditioned stimulus) with a simultaneously presented odour (conditioned stimulus). Thus, the frequency of the POR in response to presentation of an odour alone was substantially higher in locusts previously trained with paired presentations of both the odour and a food stimulus than in locusts trained with the odour alone, or with both stimuli presented separately and hence unpaired. These appetitive memories lasted for at least $24 \mathrm{~h}$, and were transferable to a different behavioural context: locusts trained on a particular odour in Pavlovian food-conditioning trials showed a subsequent preference for that odour when walking in a $\mathrm{Y}$-maze under operant conditions.

These same authors subsequently demonstrated that gregarious locusts can undergo aversive conditioning using a similar Y-maze paradigm (Simões et al., 2012). The innate olfactory preference of locusts was altered by pairing a naively preferred odour with a synthetic food to which noxious nicotine hydrogen tartrate (NHT) had been added. The concentration of NHT was sufficient to cause a variety of malaise symptoms (Bernays and Lee, 1988) without causing longterm damage. Only a single trial pairing was needed to produce robust aversive memories that lasted for at least $24 \mathrm{~h}$, and the strength of the aversion was dependent on the concentration of NHT in the food. The aversion was not immediately apparent in subsequent behavioural tests in the Y-maze however, but increased in strength over the course of $4 \mathrm{~h}$, suggesting that a postingestive mechanism was responsible for the memory formation. This was confirmed by injecting NHT directly into the haemolymph and pairing this with odour presentation. An olfactory association with the post ingestive effects of NHT could be formed with a gap of up to 30 min between injection and subsequent odour presentation, but required ingestion of neutral food simultaneously with exposure to the odour.

\subsubsection{Phase state affects aversive but not appetitive conditioning}

Naïve solitarious and gregarious $S$. gregaria have a similar 70:30 baseline preference for vanilla odour over lemon odour in a Y-maze (Simões et al., 2013). Locusts of both phases were trained by coupling a single presentation of this preferred vanilla odour with a synthetic non-nutritious food containing 10\% NHT. Just ten minutes later the locusts were given the choice between the vanilla odour and a novel lemon odour. Only $34 \%$ of solitarious locusts now chose vanilla, clearly 
indicating they had acquired a learned aversion, whereas $59 \%$ of gregarious locusts still retained a preference for the vanilla odour. The rapidity of the learned aversion in solitarious locusts suggests that it is not mediated by the post-ingestive process acting in gregarious locusts (Simões et al., 2012). By $4 \mathrm{~h}$ after training both solitarious and gregarious locusts avoided the vanilla odour stimulus. Solitarious locusts therefore form aversive olfactory memory associations much more rapidly than gregarious locusts.

By contrast, recently gregarised locusts, which had been crowded for $24 \mathrm{~h}$ prior to testing, completely failed to learn to make any aversive association between the vanilla odour and toxic food. No difference in preference from naïve locusts was seen $10 \mathrm{~min}, 4 \mathrm{~h}$ or $24 \mathrm{~h}$ post-training. Solitarious locusts crowded for more than $4 \mathrm{~h}$ are in most respects behaviourally indistinguishable from long-term gregarious locusts (Roessingh and Simpson, 1994), but Simões et al. (2012) show that their learning performance differs markedly from that of both long-term gregarious and longterm solitarious locusts.

Simões et al. (2012) also went on to show that, unlike the case with aversive learning, behavioural phase state did not affect the acquisition or retention of appetitive memories. Longterm solitarious, long-term gregarious and recently-gregarised locusts all displayed the same ability to form appetitive memories following four training trials pairing an odour with a food stimulus, and performed similarly in the Y-maze after $10 \mathrm{~min}, 4 \mathrm{~h}$ and $24 \mathrm{~h}$ post training.

Learnt memories that are acquired prior to undergoing gregarisation are not affected by the

phase-change process. Solitarious locusts that were first trained to acquire either appetitive or aversive memories retained them when they were subsequently gregarised by $24 \mathrm{~h}$ crowding. So although newly gregarious locusts are impeded from forming new aversive memories by behavioural phase change, the mechanism of memory recall is not compromised.

\subsubsection{Learning using a substance that changes from innately aversive to appetitive during phase change}

How are memories affected when a stimulus is changed from being intrinsically aversive to appetitive by behavioural phase change? Hyoscyamine is an alkaloid found in Black Henbane (Hyoscyamus niger: Solanaceae), which occurs in the natural environment of S. gregaria. Solitarious individuals avoid ingesting this plant in their diet, but recently-gregarious locusts 
actively choose to eat it (Despland and Simpson, 2005a,b). Hyoscyamine can therefore act as either an appetitive or aversive stimulus depending on a locust's phase. When artificial food containing hyoscyamine was used as an unconditioned stimulus and paired with an odour as a conditioned stimulus, solitarious locusts developed strong aversive memories to the odour, but neither longterm nor recently gregarised locusts did so (Simões et al., 2013). Hyoscyamine is therefore a negative reinforcer for solitarious locusts but neutral (and potentially positive) for gregarious locusts.

This potentially raises a problem for recently-gregarised locusts that may have formed negative associations whilst still solitarious, as memories acquired prior to gregarisation are maintained throughout the gregarisation process. How do recently gregarised locusts remove a memory to an odour induced by aversion to hyoscyamine, that is no longer useful and may prevent them eating foods that are now appetitive because the toxin they contain is now needed for chemical defence? As with NHT, the hyoscyamine-induced aversive memory is robust to gregarisation. Simões et al. (2013) showed that a single further exposure to the hyoscyamine-odour pairing post-gregarisation rapidly overwrites the previous negative association and results in a positive association instead. The blockade on aversive memory formation during gregarisation may help to prevent re-enforcement of such pre-existing memories, while the intact appetitive learning pathway would facilitate acquisition of the new positive association.

\subsection{PHASE-RELATED DIFFERENCES IN DAILY RHYTHMS OF ACTIVITY}

Gregarious S. gregaria and L. migratoria are generally day-active (diurnal) whereas solitarious animals are most active just after dusk (crepuscular: Roffey and Popov, 1968; Michel, 1970; Uvarov, 1977; Steedman, 1990; Ely et al., 2011), but this is not always the case.

\subsubsection{Solitarious locust behaviour}

In the field, solitarious adult $S$. gregaria roost in trees at night and behave cryptically during the day. They move to the ground to feed soon after dawn, shelter in low vegetation during the heat of the day and return to the ground in the late afternoon (Maeno et al., 2016). In contrast, solitarious nymphs tend to remain on their host plant throughout the entire $24 \mathrm{~h}$ period (Roffey and Popov, 
1968; K.O. Maeno unpublished observation, cited in Maeno et al., 2016). This makes sense as solitarious nymphs tend to be camouflaged against plants whereas solitarious adults are better camouflaged against desert sand. The diurnal switching of microhabitats is influenced by light, temperature, humidity and specific plant species. During the day, solitarious adults only fly when disturbed (Steedman, 1990). In warm weather they may start flying spontaneously after dusk and remain active for 1-2 h, sometimes covering large distances in relatively large numbers (Roffey, 1963; Roffey and Popov, 1968). Flights begin in response to the evening decrease in solar radiation and cease when temperatures fall below $25^{\circ}$. In the lab, they show increased walking, scanning (side-to-side movements of the head and thorax) and propensity to spontaneously take flight in the first $2 \mathrm{~h}$ after lights-off (Ely et al., 2011). Solitarious L. migratoria also fly mostly at night (Uvarov, 1977). In Mauritania solitarious adult $S$. gregaria probably feed little at night because they roost in unpalatable trees, but nymphs may feed during both night and day because they rarely move from their preferred host plant (Maeno et al., 2016).

\subsubsection{Gregarious locust behaviour}

In the field, gregarious $S$. gregaria nymphs typically roost overnight, descend to the ground before dawn (almost certainly in response to increasing light intensity), become active and march as temperatures increase, then roost again in the hottest part of the day. They then march again in the afternoon, form static groups at around sunset, and then either roost or march for several hours before roosting for the night (Ellis and Ashall, 1957). On some occasions nymphs march throughout the night. Feeding occurs at all times of the day and night, but rarely at dawn. Basking at right angles to the sun occurs relatively infrequently but generally in the morning when temperatures are low: more commonly nymphs simply cluster in warm sunny spots (Ellis and Ashall, 1957). Swarms of adults tend to take off and fly in the late morning, settle in the late afternoon (Roffey, 1963), and roost again overnight. The majority of these behavioural patterns are driven primarily by fluctuations in environmental temperature. The diurnal rhythms are, however, relatively weak: Cloudsley-Thompson (1977) states that S. gregaria are not rhythmic in the field and that their rhythms in the lab are 'feeble'. They are nevertheless described as true circadian rhythms that persist under constant dark, and which can be entrained by light and temperature. Distant approaching stimuli tend to elicit jumps during the day but hiding responses 
in the evening (H. Wilps, pers. comm. cited in Hassenstein and Hustert, 1999; but it is unclear whether this relates to solitarious or gregarious animals). In L. migratoria, entrainment to a 12:12h light: dark cycle induces a diurnal rhythm in amount of locomotion, which dies away again after a few days in constant dark (Edney, 1937; Chapman, 1954; Uvarov, 1977). The diel activity of adults differs from day to day and between individuals, but generally resembles that of nymphs. There has been no thorough analysis of diurnal patterns of activity using modern automated arena assays. In the lab, gregarious nymphs of L. migratoria feed at all times, but less so in the $4 \mathrm{~h}$ before lightson (Simpson, 1982). It is not known whether this rhythm persists under constant light conditions.

\subsubsection{Diel rhythms in emergence from the substratum}

In S. gregaria, although eggs may be laid at any time of the day or night (Ellis and Ashall, 1957; Popov, 1958), hatchlings emerge from the substratum at around dawn in both the field (gregarious phase, from at least $0.5 \mathrm{~h}$ before to $4 \mathrm{~h}$ after dawn, Ellis and Ashall, 1957) and the lab (gregarious phase, Padgham, 1981). In the lab, hatchlings from eggs laid by solitarious females also emerge from the substratum in the $4 \mathrm{~h}$ prior to dawn (Shand, 2016; see Section 7.3.5 below).

\subsubsection{Diel rhythms in hatching from the egg}

In both the field and lab, hatchlings from a single egg pod tend to hatch together, often within minutes (Ellis and Ashall, 1957; Nishide and Tanaka, 2016; Shand, 2016). This synchronicity is achieved through light, temperature or vibrational cues (Padgham, 1981; Nishide et al., 2015a,b; Nishide and Tanaka, 2016; Shand, 2016). When incubated in the lab under constant light with a $12: 12 \mathrm{~h}$ thermoperiod $\left(20: 30^{\circ} \mathrm{C}\right), S$. gregaria eggs hatch during the cryoperiod, whereas those of $L$. migratoria hatch during the thermoperiod (Nishide et al, 2015a). When diel thermal and luminance cues are both removed more than 3 days before hatching, hatching becomes arrhythmic (Nishide et al., 2015a). However, if the cues are removed only 1-2 days prior to hatching, eggs tend to hatch at around the expected time over the next 1-2 days, indicating the persistence of a weak circadian rhythm entrained to the thermocycle. There is a similar but even weaker effect in L. migratoria (Nishide et al., 2015a). Under constant temperature but with a 12:12h light: dark cycle, separated eggs of S. gregaria incubated on the substratum surface hatch during the dark phase whereas those 
of L. migratoria hatch during the light phase. The precise time of hatching is modulated by the ambient (constant) temperature, with higher temperatures leading to earlier hatching (Nishide et al., 2015b). Under these lab conditions, eggs buried in sand hatch arrhythmically, so the authors conclude that light may not be an important zeitgeber (external cue that entrains a circadian rhythm) in the field; but light intensities in the incubator (200 lx) were 5000-fold lower than intense daylight $\left(10^{5} 1 \mathrm{x}\right)$.

In the cricket Gryllus bimaculatus, the timing of egg hatching is influenced by an internal circadian clock that is active by about mid embryogenesis and which thereafter entrains to photoperiod (Tomioka et al., 1991). The photoperiodic conditions to which the parents were exposed can also influence the timing of hatching. So far, the data for locusts are less clear: there is a suggestion of weak circadian control of hatching in S. gregaria, but no such evidence for $L$. migratoria. Further experiments with natural zeitgebers would be useful.

\subsubsection{Phase differences in hatching and emergence}

In the lab, the eggs of solitarious $S$. gregaria hatch approximately $2 \mathrm{~h}$ later than those of gregarious animals when maintained under constant light with a 12:12 h thermoperiod and placed separately on or just under the surface of the substratum. No such difference is observed in L. migratoria (Nishide et al., 2015a). Under these conditions, however, hatching is very spread out during the cryophase (S. gregaria) or thermophase (L. migratoria), so it is difficult to relate these observations to the normal situation in the field where eggs hatch synchronously at around dawn. It is not known whether similar phase differences are observed for separated eggs under a 12:12 h photoperiod with constant temperature.

Emergence of $S$. gregaria nymphs from natural egg pods laid in sand in the lab and incubated under coincident 12:12 $\mathrm{h}$ thermo- and photoperiods differs between the phases (Shand et al., 2015; Shand, 2016). Those of solitarious parents emerge on average 40 min earlier than those of gregarious parents (57 min vs 17 min prior to dawn). Importantly, eggs of solitarious

parents placed individually under a 1-2 mm thick layer of sand and incubated under the same 12:12 $\mathrm{h}$ thermo- and photoperiod hatched $42 \mathrm{~min}$ later than similarly treated eggs of gregarious parents (2:12 h h vs 2:54 h prior to lights on, Shand, 2016), which is consistent with Nishide et al. (2015a). 
It thus appears that under natural conditions eggs of solitarious $S$. gregaria may hatch later but emerge from the substratum earlier than those of gregarious animals.

\subsection{NEUROPHYSIOLOGICAL CORRELATES OF DIEL PATTERNS OF ACTIVITY}

Pener and Simpson (2009) reviewed the relatively few studies of neural differences between the phases available at that time. Matheson et al. (2004) and Rogers et al. (2007) demonstrated phase related differences in the descending contralateral movement detector (DCMD) visuo-motor pathway, while Fuchs et al. (2003) demonstrated differences in the tritocerebral commissure giant (TCG) sensory-motor pathway involved in flight in S. gregaria. The tritocerebral commissure dwarf (TCD) is a GABAergic interneuron in the same sensory-motor pathway that is sensitive to ambient illumination intensity (Tyrer et al., 1988). The spontaneous activity of the TCD was greater under dark compared to light conditions in both phases, but the dark-related activity was five times higher in gregarious locusts than in solitarious locusts, suggesting that there is far greater synaptic inhibition onto the flight circuitry under dark conditions in gregarious locusts (Fuchs et al., 2003).

Rogers et al. (2010) have built on the earlier DCMD work by showing that solitarious $S$. gregaria have larger eyes located differently on the head and greater binocular overlap than gregarious animals. The receptive field of DCMD to looming stimuli is broadly similar in shape and sensitivity in the two phases, but there is a small caudolateral region of enhanced sensitivity in gregarious animals. Gregarious locusts habituate less to repeated looming stimuli along the eye equator, as previously reported, but there is no difference in rate of habituation in other parts of the receptive field. Rogers et al. (2010) suggest that the different DCMD receptive field properties may reflect the need for diurnal gregarious and nocturnal solitarious locusts to escape from different types of predators. Gaten et al. (2012) show that the responsiveness of DCMD to looming objects varies in a true circadian rhythm, and that the pattern differs in solitarious and gregarious S. gregaria. The strength of response varies sinusoidally with a period close to $24 \mathrm{~h}$ under constant light in solitarious locusts, but in gregarious locusts the 24 h rhythm is modified by secondary ultradian rhythms. The strongest responses occur at around expected dusk in solitarious locusts, but some $6 \mathrm{~h}$ earlier in gregarious locusts, thus matching the times of day at which locusts of each type are most active. Shand (2016) shows that overall eye sensitivity measured using an 
electroretinogram (ERG) varies in a very similar diurnal pattern in solitarious and gregarious $S$. gregaria, with little or no evidence for a true circadian rhythm. The peak of sensitivity is after dusk in both phases, which means that the robust difference in time of peak sensitivity in DCMD reported by Gaten et al. (2012) must originate downstream of the photoreceptors.

Gordon et al. (2014) show that adult solitarious S. gregaria are more sensitive than gregarious animals to high frequency sounds, and relate this to the need for nocturnal solitarious locusts to avoid bat predators. The work does not, however, attempt to determine if there are diel changes in auditory sensitivity.

A key area where research is lacking is in an understanding of the functioning of central clock neurons in any locust.

\subsection{CIRCADIAN CLOCK GENES IN SCHISTOCERCA GREGARIA}

Clock genes regulate the function not only of neurons that comprise the central circadian clock, but also the functions of cells and tissues throughout the body (reviewed for insects by e.g. Tomioka and Matsumoto, 2010). Tobback et al. (2011) produced partial sequences for the core clock genes clock, period and timeless from an existing database of $S$. gregaria expressed sequence tags (EST). Both gregarious and solitarious locusts show the same diel pattern of expression of these three genes in animals maintained under a 14:10 h light:dark cycle. The authors describe this as a 'circadian' rhythm, but since animals were not examined under constant dark, this term is not justified. Clock is highly expressed in the brain at lights-on, whereas period and timeless both have double peaks at 12 and $18 \mathrm{~h}$ later, with a dip at $15 \mathrm{~h}$. This strange pattern may be related to the 14 h photoperiod (Tobback et al., 2011). The similarity of the diel patterns seen in solitarious and gregarious locusts suggests that observed differences in diel patterns of behaviour are regulated downstream of the molecular clock.

Tobback et al. (2011) used RNAi to knock down expression of period and timeless in gregarious female $S$. gregaria, but this resulted in reduced expression (mRNA levels) only in ovary, and not in brain. The knockdown in ovary resulted in females laying fewer eggs. RNAi of clock was lethal in females prior to sexual maturity. Tobback et al. (2012) performed RNAi against period and timeless in gregarious male $S$. gregaria, which resulted in reduced expression only in testes and accessory glands, and not in brain. Untreated females mated with dsRNAi treated males 
produced fewer fertilised eggs, possibly because of disrupted diel patterns of sperm transfer in the male reproductive organs.

There has been no comparative study of the molecular biology of the circadian clock in any locust.

\section{Collective behaviour}

\subsection{LABORATORY STUDIES OF LOCUST COLLECTIVE BEHAVIOUR}

Animal collective behaviour, specifically the emergence of synchronization among moving groups of animals, is an important ecological phenomenon (Sumpter, 2010). The vastness of locust swarms, and their severe impact on both the natural environment and human agriculture makes them a particularly important example.

In recent years there have been several new attempts to describe or capture locust collective behaviour using theoretical modelling, as part of the long-lasting endeavour to understand the locust phenomenon. A recent review on locust collective motion by Ariel and Ayali (2015) focussed mostly on theoretical and modelling efforts to explain the emergence and maintenance of order in marching locust nymphs. While flying swarms of adults may attract general attention, the marching bands of nymphs, beyond constituting a critical stage in the development of locust aggregations, also demonstrate remarkable synchronization and coordination. Some modelling studies are closely associated with biological data and observations, which aim to specifically explain locust behaviour, while others offer a more abstract mathematical or physics-based perspective (Ariel and Ayali, 2015). This dualism can be found in many locust studies and reflects the open question of how unique an example of collective movement is locust swarming, and what general lessons are to be learned from it?

\subsubsection{Locust swarming and coordinated movement}

Aggregation, the formation of dense groups of nymphs, is a major behavioural characteristic of gregarious locusts that appears soon after hatching. It should be noted, however, that locust 
aggregation per-se differs from coordinated movement. The latter, referred to as marching behaviour, also appears a few days after hatching, and continues to constitute a dominant part of the nymphs' behaviour until adulthood, when they begin to fly.

Collective marching is affected by environmental conditions (e.g. temperature, rain, wind, and topography; Uvarov, 1977; Amichay et al., 2016), but it is a self-emergent process, induced by local interactions among the locusts within the group. The nature of these local interactions is a major focus of research.

Undisturbed nymphs within marching bands will advance by means of persistent walking, intermittently interrupted by pauses (Uvarov, 1977; Ellis and Ashall, 1957; Ariel et al., 2014). The speed of walking varies, as do the durations of the walking or pausing bouts. Locusts at the edges of the swarm will alter their behaviour, which maintains the integrity of the band: e.g. locusts at the front will slow down or turn more often, reducing their net displacement in comparison to those behind (Uvarov, 1977). The major factors that affect the general direction of marching are still unclear. There may well be fundamental species-related differences in the interaction of the marching band and the environment (as well as in other aspects of the collective behaviour), reflected in differences in the overall shape of the bands (Uvarov, 1977; Hunter et al., 2008; Sumpter, 2010).

Relatively few studies have focussed on the local dynamics within flying swarms due to obvious technical limitations, though see Edelstein-Keshet et al. (1998) for a mathematical approach. Flying swarms are assumed to migrate predominantly down-wind (Cooter, 1989). Interestingly, and somewhat similar to the case of the nymphs at the edge of the marching band, locusts flying out of the migrating swarm will turn back to re-join it.

\subsubsection{Laboratory studies of locust collective movement}

Recent progress in our understanding of the rules governing animal collective motion has been made possible by the development of new methods for generating coordinated motion under controlled lab conditions and reliably tracking individuals within collectively moving groups. Several studies have revisited the pioneering work of Ellis and colleagues (e.g. Ellis, 1959, 1963a,b), investigating marching behaviour in closed circular arenas. Lab-based manipulations of locust density are now accompanied by automated video tracking and innovative statistical 
methods, which have yielded important insights into large-scale characteristics of locust swarms and the rules governing collective motion, as well as into the local animal-animal interactions or the details of locust motion within the crowd.

Buhl et al. $(2006,2011)$ demonstrated that locust collective movement is highly dependent on group density and is mediated by combinations of social interactions such as avoidance, alignment, and attraction. These findings spurred later modelling studies. Ariel et al. (2014) reconfirmed that a dominant feature characterizing locust behaviour is their intermittent, pauseand-go, walking pattern. This finding, previously reported by Bazazi et al. (2012) for a single animal, was shown to persist in all individuals within a marching band. It was suggested that the coordinated marching behaviour of the locust swarms is manifested by the repeated decisions taken by individual animals to initiate or resume walking. These recurring decisions can be explained by sensory stimuli conveyed by the optical flux in the vicinity of the individual, due to the behaviour of others in the crowd (Ariel et al., 2014), supported by the locusts' high propensity for walking. Recent technological advances in achieving high accuracy and reliable tracking of all individuals in the arena promise to further augment our knowledge by way of carefully planned laboratory experiments.

\subsubsection{Modelling locust collective behaviour}

Locust collective movement occurs across different spatial and temporal scales -from the individual ( $\mathrm{cm}$ and minutes) all the way to swarms comprising millions of insects ( $\mathrm{km}$ and hours). Mathematical models of these phenomena tend to assume a homogeneous population, in which the influence of conspecifics on each individual is subject to some local average (or weighted average). While these assumptions may be somewhat unrealistic from a biological perspective, recent models have nevertheless predicted some important aspects of locust behaviour.

Most theoretical work on locust collective movement has established either continuousbased models, which define broad patterns of interactions across the entire group, or individualbased models, which focus on the local interactions of single locusts with their neighbours. Yates et al. (2009) and Escudero et al. (2010) used continuous models to determine that the mean time between directional changes increased exponentially with group density. Escudero et al. (2010) also established that direction switches are an inherent property of collective movement, and that 
their precise timing is unpredictable. This work was expanded upon more recently by Dyson et al. (2015), who combined empirical data from Buhl et al. (2006) with an individual-based model to show that interactions between individuals in marching bands of S. gregaria occur with at least two neighbours simultaneously.

Examples of individual-based models include that of Buhl et al. (2006), which was a SPP (self-propelled particles) model adapted from Czirók et al. (1999; based in turn on Vicsek et al., 1995). The main feature of such models derives from the understanding that the macroscopic state of the system can be classified into one of two well-defined phases (in the sense of statistical physics): order and disorder. Switching between the phases requires an external change in the system parameters such as concentration or the amount of noise. This finding introduced the possibility that order is closely dependent on the number of individuals, as demonstrated in the laboratory experimental arena.

A specific class of two dimensional SPP model is termed Escape and Pursuit (E\&P; Romanczuk et al., 2009). This modelling approach, directly motivated by experimental work (Bazazi et al., 2008, 2011) postulated that marching locusts are continuously escaping from conspecifics behind them while at the same time pursuing others in front of them. The strength of this model is the suggested direct link between the local individuals' interactions and the coordinated motion of the swarm. However, this modelling approach was questioned by Ariel and Ayali (2015), who note that most observations of locust behaviour are not consistent with aggressive interactions between healthy individuals within the marching band (cannibalism is far more common where the cannibalised individual is injured or dead). This observation is not necessarily inconsistent with a role for cannibalism in shaping these collective behaviours, precisely because the strategies locusts might have evolved to avoid direct contact with one another are successful, and reduce the risk of antagonistic interactions to such a level that it is outweighed by the group-related benefits of reduced predation risk and the possibility of future cannibalism-derived nutrients (Hansen et al., 2011). This interesting debate would benefit from field studies, of which Buhl et al. (2012) is presently the only recent example. Investigating a swarm of $C$. terminifera, the authors reported a tendency for locusts to interact with all their surrounding neighbours. There was also evidence of a short-range repulsion force, balanced by a clustering force, similar to what has been observed in a repulsion/ alignment/ attraction-based SPP model. This study rejected the E\&P mechanism for $C$. terminifera where marching had already 
been established (as opposed to during its onset) but, given that this is only one study in one species, further fieldwork would clearly be highly beneficial. The extent to which cannibalism is observed (or not) in extant populations does not discount a role for this behaviour in the evolution of collective movement (Guttal et al., 2012, and see details in Section 2).

An alternative approach to the E\&P model was suggested by Ariel et al. (2014). Further placing the emphasis on the individual animal and following their own experimental observations of locusts marching in an experimental arena, they base their model on a set of discrete decisions - when to stop, walk, or turn, rather than a continuous process. Here the calculated local averages (as well as the order parameter) consider only the walking individuals. This model effectively captures the basic behaviours of locusts in a marching band, in addition to offering testable predictions.

\subsection{THE NEED FOR LARGE SCALE MOVEMENT DATA}

Laboratory experiments (Ellis, 1951, 1953, 1964a,b; Buhl, 2006; Ariel et al., 2014) and minimal collective movement models (Vicsek et al., 1995; Buhl et al., 2006; Ariel et al., 2014) have helped establish that local interactions between individuals, for example alignment with nearest neighbours, are the fundamental elements underpinning locust collective movement. Collective movement is a central and defining part of the behavioural repertoire of gregarious locusts, but key questions remain concerning the benefits of collective movement to locusts, and how it originally evolved. Current models have barely started addressing these questions. Benefits to animal groups include: protection from predation by a dilution effect or through enhanced collective escape responses (Hamilton, 1971; Foster and Treherne, 1981); improved navigation capabilities such as gradient climbing (Sumpter, 2010); and increased migration survival due to the potential for cannibalism (Hansen et al., 2011; though see discussion in Section 8.1.3). All these functions are either directly dependent on group structure, or likely to be strongly influenced by variations of density within the group, its structure and dynamics. Yet, current models are unable to account for the large-scale group structures observed empirically (Sumpter, 2010). Locust migratory bands exhibit a wide range of patterns from "columnar formations" (Uvarov, 1977), such as the intertwined narrow and dense stretching streams of L. pardalina (Uvarov, 1977), to "frontal formations", such as wide crescent shaped frontal formations followed by looser comet- 
like tails of C. terminifera (Clark, 1949; Uvarov, 1977; Hunter et al., 2008; Buhl et al., 2011). Some species, such as D. maroccanus can alternate between both types of formations (Uvarov, 1977). There is a need to address locust collective marching at the large scale at which nymphal bands occur under natural conditions. While modelling techniques such as parallel computation using general-purpose computing on graphics processing units (GPGPU) make it theoretically possible to simulate nymphal bands at sufficiently large scales (e.g. Erra et al., 2009; Torney et al., 2011), gathering the relevant quantitative empirical data from the field remains a major challenge.

\subsubsection{Studying nymphal band patterns and their movement over their lifetime}

Quantifying and mapping locust nymphal bands in the field is time consuming and detailed data are still extremely rare and limited to a few species (e.g. in S. gregaria: Ellis and Ashall, 1957; Ashall and Ellis, 1962; N. septemfasciata: Dean, 1967; the outbreaking Gomphocerine Mato Grosso Grasshopper, Rhammatocerus schistocercoides: Lecoq et al., 1999; Lecoq and Balanca, 1998; see also Uvarov, 1977, for figures, photos and descriptions of bands in other species). While there are not enough data to determine which factors lead to the large variation of nymphal band patterns observed both within and across species, these studies reveal some key features specific to locust nymphal bands compared to other collectively moving animals. Locust bands tend to move at much slower speeds than the individuals within it. This is partially explained by individual locusts tending to move in short bursts of locomotor activity (in the field: Ellis and Ashall, 1957; Uvarov, 1977; observed in laboratory experiments by Ellis, 1953; Buhl et al., 2006; Ariel et al., 2014) but also because nymphal bands are a product of both aggregation and marching. Nymphs exhibit aggregation behaviour in three main contexts. First, they form dense clusters overnight or when the temperature is too cold to march, which occur in vegetation or on the ground. Second, they climb up vegetation and aggregate when the ground temperature becomes too hot (perching). Third, they aggregate either on the ground or in vegetation when they reach the front of their band (Uvarov, 1977). The alternation of marching and inactivity at the individual level is also likely to be influenced by nutritional state. Locusts undergo well marked internal rhythms linked to their feeding activity (Simpson, 1981) and need to accommodate their own nutritional state with the need to move and remain within the group. An in-depth study of the link between individual behaviour and emergent nymphal band patterns will require the ability to systematically track and 
quantify patterns and movement at both individual and group levels, which still poses significant technical challenges.

\subsubsection{Remote piloted aircrafts and nymphal bands and vegetation photogrammetry}

Even with the use of global positioning system (GPS) units, ground surveys remain too timeconsuming to map small nymphal bands several times per day, let alone large ones. Bands can be visible during aerial survey operations, but such surveys are both expensive and not always capable

of detecting smaller bands when relying on conventional manned aircraft operated at safe altitudes above 100 m (Hunter et al., 2008). Unmanned Aerial Vehicles (UAV; also known as "drones" or Remotely Piloted Aircraft Systems (RPAS)) promise to revolutionise surveying because they are both cheap to operate and typically fly at low altitudes, below $100 \mathrm{~m}$. While their application to the surveying and studying of locust nymphal bands is at an early stage of development, UAVs are increasingly used in ecological and conservation contexts (Koh and Wich., 2012; Marvin et al., 2016). Their use ranges from monitoring areas using live video feedback; to mapping areas of study using high resolution camera images (either conventional RGB or thermal, multispectral, hyperspectral) which can be stitched into larger pictures using photogrammetry; to remotely detecting sensors carried by animals. All of these applications could be adapted for use on locust nymphal bands.

Both main types of UAV, multi-rotor and fixed-wing aircraft, are suitable for surveying or mapping locust nymphal bands. For locust work, the main immediate limitation often comes from existing aviation regulations that insist that UAV operators keep their aircraft within visual line of sight (VLOS), which effectively imposes a flight radius of approximately $500 \mathrm{~m}$. Within such ranges, most UAVs are easily capable of overflying a locust band at altitudes ranging from 50 to100 $\mathrm{m}$ and recording overlapping pictures suitable for photogrammetry at resolutions of up to a few $\mathrm{cm}$ per pixel within 20 minutes. Where flying beyond visual line of sight (BVLOS) is allowed, fixed wing UAVs can have the advantage of having a much longer flight time (up to an hour) and a larger range (up to $25 \mathrm{~km}$ in Koh and Wich, 2012) but the operational and training requirements are greater.

\subsubsection{Individual movement quantification and tracking in the field}


Tracking the movement of individuals remains challenging. Some studies have quantified individual movement over relatively small scales, starting as early as the 1960s with the pioneering work by Stower (1963), and more detailed recent papers have managed to quantify some movement characteristics and infer interaction rules (Buhl et al., 2012). For larger spatial and temporal scales, insect-borne sensors, including very small ones, are becoming increasingly accessible. The most promising tracking method uses harmonic radar transponders, such as those used to track individual bees (Riley et al., 2005; Lihoreau et al., 2012) or Mormon Crickets, Anabrus simplex (Sword et al., 2005). Recent progress in transponder miniaturisation is bringing these technologies within a size and weight range that would allow them to be fitted onto final larval instar C. terminifera (Tahir and Brooker, 2015). The radars required to track these sensors used to be too large to be frequently transported, but Frequency Modulated Continuous Wave (FMCW) radars operating in the millimetre wave band will soon be small enough to be carried as UAV payloads (Tahir and Brooker, 2015).

\subsubsection{Conclusions}

Existing quantitative field data is still extremely scarce, and more is critically needed to understand the emergence of the large-scale patterns produced by locust nymphal bands. Recent advances in both the development of UAV and miniature sensors will allow the gathering of data at unprecedented spatial and temporal scales, which should continue to be combined with laboratory, modelling and theoretical approaches. Furthermore, and given that collective behaviour is a crucial aspect of the locust phenotype, these varying approaches should be employed across a range of locust species, with a view to offering new perspectives for evolutionary and phylogenetic studies (see Section 2).

\section{$9 \quad$ Environmental stimuli affecting phase change}

Locust phase change can be induced to a greater or lesser extent through some combination of visual, olfactory and tactile stimuli from other locusts. As discussed in Section 4, there is some 
evidence that olfaction plays a role in phase change of L. migratoria, and it also causes phase change in S. gregaria in combination with visual stimuli from other locusts (Roessingh et al., 1998). Tactile stimulation of the hind femur is also a potent gregarising factor in $S$. gregaria (Simpson et al., 2001), while tactile stimulation of the antennae has the same effect in $C$. terminifera (Cullen et al., 2010). It should therefore follow that any environmental variables that bring locusts into close proximity, and especially those that increase direct contact between individuals, will generally promote gregarisation. At a small scale, clumped resources promote interactions and subsequent gregarisation among S. gregaria (Bouaïchi et al., 1996; Collett et al., 1998; Despland et al., 2000; Despland and Simpson, 2000; Babah and Sword, 2004). In contrast, dispersed resources tend to keep locusts spread apart from each other, thereby maintaining them in the solitarious phase. Using survey data from routine monitoring from 2003 to 2011 in Mauritania, Cissé et al. (2013) used a statistical model to show that sparse and dry vegetation lowered the threshold of gregarisation relative to dense and green vegetation, presumably by increasing locust interactions. By analyzing gregarious behaviour in circular arenas in the field, Cissé et al. (2015) corroborated those results showing that when at similar densities for a given $100 \mathrm{~m}^{2}$, locusts collected from areas with a lower percent vegetation cover tended to be more gregarious. However, other vegetation parameters such as greenness and distance between plants were found to be poor predictors of phase status. In a subsequent study, Cissé et al. (2016) used field assessments of locust phase state (predominantly clumping behaviour) collected in 2012 and 2013 to test the predictions of the Cissé et al. (2013) statistical model. The model predicted the phase of field populations of $S$. gregaria with only a $6 \%$ error rate. Using an occupancy model, Veran et al. (2015) showed that there was a higher probability of scattered populations leading to gregarious outbreaks of $C$. terminifera in desert areas as compared to grasslands. This pattern may be due to resources being more clumped in the desert. Despland and Simpson (2000) found an interaction between food quality and distribution; S. gregaria have an increased amount of direct contact, and are therefore more likely to gregarise, if they have to regularly switch among food sources that have complementary nutrient contents. Bazazi et al. (2011) found that crowd-reared S. gregaria nymphs fed low-protein diets for two days had an increased walking speed, which likely translates to increased interactions and the formation of migratory bands. The effect of resource distribution on gregarisation likely differs by scale. In contrast to studies focussed at a small-scale, a large-scale landscape study using satellite imagery showed that S. gregaria 
outbreaks tended to occur when vegetation was more fragmented as opposed to clumped (Despland et al., 2004). These studies illustrate that resource abundance, quality and distribution are all important factors influencing the likelihood that locusts will gregarise. If these same mechanisms apply to species beyond $S$. gregaria and $C$. terminifera, and why it might be functionally beneficial for locusts to undergo behavioural phase change under these different conditions, are still open questions.

Limited experiments have explicitly tested the major evolutionary drivers of locust phase change, but it is likely a complex set of interacting factors arising from living in a dynamic and unpredictable environment that differ to some extent among species (see Section 2). At the outbreak scale of localised populations (prior to a larger upsurge or plague), the benefits of some phase-related traits have been clearly demonstrated above a certain population density threshold. For example, behavioural phase change that leads to aggregations when at high density is likely an anti-predator strategy. The appearance of aposematic colouration only at high density fits with warning colour theory, which predicts this strategy to be beneficial above a critical density of individuals expressing similar colouration (Sword, 2002). High density-induced colour changes have been shown to signal toxicity (Sword, 1999). In S. lineata, high-density animals feed on toxic Wafer Ash, Ptelea trifoliata, which causes them to gain "gut-content mediated toxicity" to vertebrate predators (Sword, 1999; Sword, 2001). Similarly, solitarious phase S. gregaria avoided foods containing the alkaloid hyoscyamine, while gregarious locusts did not (Despland and Simpson, 2005a). When solitarious locusts were subsequently crowded, they began to accept food containing hyoscyamine. Even in cases where insects lack aposematic colouration or plant-derived toxicity, there can be anti-predator advantages to being a part of the crowd. Sword et al. (2005) tracked Mormon Crickets, A. simplex in the field and found that those marching in a crowd had lower levels of predation than those not within the crowd. Crowd-reared locusts tend to have a faster development rate (Pélissié et al., 2016), which likely decreases susceptibility to predation during the nymphal stages.

Once at high population density, locusts increase activity levels and may migrate en masse due to a combination of factors that may include the threat of cannibalism (Bazazi et al., 2008; Guttal et al., 2012), decreased disease transmission (Altizer et al., 2011), and/or seeking better resources. Locust phase change is often (but not always) coupled with migratory swarms (Key, 1950; Sword, 2003). Thus, it has been hypothesised that high population density increases 
intraspecific competition for food, and migration towards further resources by gregarious phenotypes at least partially mitigates the effects of this competition (Johnson, 1969). Indeed, many traits associated with phase change support the broad hypothesis that gregarious locusts are migratory, and solitarious locusts are sedentary phenotypes. The forewing length:hind femur length ratio is higher in gregarious than solitarious adults of $S$. gregaria, $N$. septemfasciata, and $L$. pardalina (Uvarov, 1966 and references therein; see also Nolte, 1967). Presumably, increased values are correlated with decreased wing loading (wing area/body mass) and higher flight capacity, although experimental tests are limited. In addition to changes in morphometry that enhance migratory capacity, gregarious locusts tend to have a proportionally higher resting metabolic rate (Butler and Innes, 1936; Uvarov, 1966; Heifetz, 1997; Applebaum and Heifetz, 1999; Cease et al., 2010), perhaps due to the elevated maintenance cost of larger flight muscles (Nespolo et al., 2008). Gregarious locusts also tend to have increased lipid stores for long-distance flight, and a delayed onset of fecundity (Uvarov, 1966; Hunter et al., 1981; Pener et al., 1997; Pener and Yerushalmi, 1998; Applebaum and Heifetz, 1999), which supports a general model of increased investment in flight-related physiology and morphology at the expense of reproductive capacity. Even as hatchlings, gregarious S. gregaria have higher body lipid contents than solitarious hatchlings (Maeno et al., 2013). The increased energy reserves made gregarious hatchlings less susceptible to starvation and would presumably also increase marching capacity. Density-dependent polyphenism has been documented even in acridids that do not form agriculturally damaging swarms. For example, the Oedipodine Aiolopus thalassinus has never been recorded to march or swarm, but does develop more quickly and have increased activity levels, haemolymph lipids and carbohydrates, metabolic rate, and food consumption compared to those of solitary-reared insects (Heifetz and Applebaum, 1995). Such examples support the concept that different reaction norms of locust phase polyphenism have evolved separately from each other (Song, 2005; Song and Wenzel, 2008).

Because locust phase polyphenism is often, but not always, coupled with migration (Pener, 1991), it has been hypothesised that poor resources should promote phase change (Johnson, 1969). Nutrition is influential in regulating migratory plasticity in other insects (e.g. Denno et al., 1985; Müller et al., 2001; Dixon, 2012) and, in the short term (two days), Bazazi et al. (2011) found that $S$. gregaria fed low-protein diets increased walking speed in gregarious nymphs. In a longer-term study, Maeno and Tanaka (2011) found that S. gregaria reared on wilted grass had higher wing: 
leg length ratios, but decreased performance, relative to locusts reared on fresh cut grass. However, nymphs were reared individually and more experiments are required to determine if gregarious, crowd-reared S. gregaria respond in the same way. In contrast to the hypothesis that poor resources should enhance development of migratory characteristics (Johnson, 1969), new evidence suggests that nutritionally-optimal diets may be required to promote increased migratory capacity, at least in some species. Cease et al. (2017) found that Mongolian Locusts, Oedaleus asiaticus reared at high population density and fed low-nitrogen grass (nutritionally optimal for this species) had increased wing area and decreased wing loading relative to locusts fed high-nitrogen grass (which decreases growth and survival in this species; Cease et al., 2012). The authors then reared locusts on three synthetic diets differing only in the protein: carbohydrate ratio for the duration of the $5^{\text {th }}$ instar and measured flight behaviour in the adults. Locusts fed their nutritionally-optimal dietary ratio of 1:2 protein: carbohydrate flew for longer than locusts fed imbalanced diets with lower or higher protein:carbohydrate ratios. Faki (2004) found that S. gregaria reared on no-choice diets of their preferred host plants Pennesitum typhoideum or Heliotropium undulatum tended to have morphometrics more closely aligned with gregarious locusts as compared to S. gregaria reared on a less-preferred and presumably less nutritionally-optimal Aerva javanica. These studies suggest that a locust's nutritional state, and wider nutritional landscape, might directly influence its reaction norm for migratory capacity in response to population density, in addition to the described indirect effects of resource variation on phase change (Despland et al., 2000, Despland and Simpson, 2000). Other environmental factors may also modulate the effect of density by altering the threshold at which an individual locust responds to crowding stimuli. There is some evidence to suggest that the microsporidian Paranosema locustae might suppress phase change in $L$. migratoria migratorioides, although it is unknown if this was a direct effect or if it was behaviourally-mediated by slowing locusts down and therefore decreasing their interactions with conspecifics (Fu et al., 2010; species given as L. migratoria manilensis). These studies suggest a pattern whereby conditions that favour population growth may also favour development of gregarious phenotypes. Population growth often leads to increased effective density, which is the primary trigger of phase change. Therefore, it seems plausible that conditions promoting population growth might not suppress, but instead promote, gregarisation. Moreover, access to high quality food resources may be necessary to support the energetic demands of the gregarious phase. Non-optimal diets may make it difficult for locusts to achieve the physiological condition 
necessary for successful migration and may preclude migration en masse even when faced with intraspecific competition under crowded conditions. This area of locust research supports recent calls for a renewed study of migratory and dispersal reaction norms (Clobert et al., 2009) to aid in developing an understanding of the regulation and evolution of migratory and dispersal behaviours (Burgess et al., 2015).

\section{Ecology and landscape-level processes}

\subsection{REMOTE SENSING}

The immense, often transcontinental scale of potential locust habitats requires fast and efficient monitoring dictated by meteorological conditions and locust life cycles. Locust surveying is a daunting and challenging task as locust habitats often cover vast, remote and transborder areas with difficult and insecure access. Traditionally, it is performed by ground survey teams, who collect locust information and assess ecosystem parameters with particular attention to vegetation. Such ground-based surveying is often incapable of providing fast and reliable information on spatio-temporal distribution of potential locust habitats. Remote sensing in the form of satellite imaging appears to be an appropriate tool for addressing the challenges of scale and time in locust monitoring.

The first attempts of using information from satellites in locust monitoring were implemented by Pedgley (1974) and Hielkema (1981). Subsequently Tucker et al. (1985) recognised the potential of the remotely sensed data for $S$. gregaria surveying and forecasting. Satellite images, particularly from Landsat, were used to map vegetation in the gregarisation areas of S. gregaria in West Africa (Hielkema et al., 1986; Ghaout, 1990; Loveaux et al., 1990). In Australia, satellite data were first used by McCulloch and Hunter (1983) to inventorise $C$. terminifera habitats. The authors classified Landsat images and found that locust presence was confined to three ground cover classes: stony downs, stony plains, and high-elevation floodplains. Further development of remote sensing tools in Australia was done by Bryceson and colleagues, who demonstrated that it is possible to detect even small areas of vegetative growth after rains with Landsat data (Bryceson, 1984; Bryceson and Wright, 1986). Subsequently, Landsat images 
were instrumental in identifying oviposition beds (Bryceson, 1989) and the source areas from which plagues of C. terminifera developed (Bryceson, 1990, 1991). Remote sensing data are currently incorporated in a decision support system used by the Australian Plague Locust Commission (APLC) in its routine forecasting and management activities (Deveson and Hunter, 2002; Deveson, 2013).

Early detection of potential $S$. gregaria habitats became possible with the development of the Normalized Difference Vegetation Index (NDVI), a remote sensing technique which allows observers to distinguish areas of green vegetation from bare soil. Cherlet and Di Gregorio (1993) attempted to calibrate the NDVI delivered by the Advanced Very High Resolution Radiometer (AVHRR) satellite of the National Oceanic and Atmospheric Administration of the USA (NOAA), using extensive ground-collected field data from Niger. Voss and Dreiser (1994) used the NDVI to detect vegetated areas in Mauritania. Dreiser (1994) used Landsat data to produce $S$. gregaria habitat maps for certain areas of Sudan, Mali and Mauritania. Landsat data, which have a 16-day temporal resolution, were deemed adequate to assess the potential suitability of $S$. gregaria habitats. However, the identification of the actual habitats required higher temporal resolution imagery like that provided by the NOAA AVHRR satellite, in combination with meteorological data from Meteosat and other similar satellites (Voss and Dreiser, 1997).

Various remote sensing techniques have been used on a regular basis for Desert locust monitoring by Food and Agriculture Organization's Desert Locust Information Service (FAODLIS) in Rome since the 1990s. Early detection of habitat conditions favorable to locust outbreaks is a key element in locust preventive strategy, which relies on finding $S$. gregaria populations before they can expand into full-scale plagues (Magor et al., 2008). Satellite imagery is used to characterise changes in vegetation cover and soil moisture in order to direct ground survey teams to areas with the highest probability of conditions conducive to $S$. gregaria breeding. The first satellite imagery used in FAO-DLIS early warning systems was Meteosat cloud imagery, which made it possible to pinpoint rainfall areas (Cressman, 1996). A few years later, the NDVI delivered by NOAA AVHRR sensors with $7.6 \mathrm{~km}$ spatial resolution improved our understanding of locust habitats. Since then, the spatial resolution of satellite imagery has continued to improve, along with the treatment algorithms, which has greatly aided the identification of potential and actual $S$. gregaria habitats from West Africa to India through the estimation of rainfall and vegetation cover (Cressman, 2013). Since the 2000s, the FAO-DLIS has used Moderate Resolution Imaging 
Spectroradiometer (MODIS) images at a spatial resolution of $250 \mathrm{~m}$, consisting of a 16-day composite image of NDVI values. Starting from 2010s, the FAO-DLIS has incorporated dynamic greenness maps derived from the NDVI index from MODIS data (Pekel et al., 2011; Renier et al., 2015). In addition, daily, 10-day and monthly rainfall estimates are produced on a $0.25^{\circ} \times 0.25^{\circ}$ latitudelongitude grid (Dinku et al., 2010). These various remote sensing data are incorporated into a Geographic Information System (GIS) called RAMSES (Reconnaissance And Monitoring System of the Environment of Schistocerca) allowing national locust information services to manage, query, display, analyze, and map field and other data (Cherlet, 1993; Cressman, 2013). MODIS-derived NDVI is also currently used in Australia for locust forecasting (Deveson, 2013) although some specialists question its predictive capacity (Weiss, 2016)

Green vegetation emerging after rains in the desert is known to play a crucial role in the initial concentration and gregarisation of S. gregaria (Despland, 2003). Furthermore, the fractal dimension of vegetated areas, which measures its degree of spatial fragmentation, was demonstrated to be positively related to the gregarisation potential of $S$. gregaria populations (Despland et al., 2000). The authors used NDVI pixels of $7.6 \mathrm{~km}$ over a vegetation threshold in good and bad reproduction periods, across an area of approximately $500 \mathrm{~km}$ x $1300 \mathrm{~km}$. However, Piou et al. (2013) did not find fractal dimension to be a good indicator of locust presence when analysed at a finer spatial resolution of $250 \mathrm{~m}$ and across a smaller area. Tratalos and Cheke (2006) also argued that NDVI data derived from satellite images covering large areas (from 4 to $8 \mathrm{~km}$ spatial resolution) were not a good predictor of $S$. gregaria presence. However, Piou et al. (2013) did find that NDVI at $250 \mathrm{~m}$ resolution can be a good predictor of $S$. gregaria occurrence. These studies support the potential of satellite imaging to identify habitats susceptible to locust invasions, which is critical for early warning and preventive management (Cressman, 2013). Predicting presence of locusts can be improved if remote sensing information and vegetation indices are coupled with historic survey data (Deveson, 2013; Piou et al., 2013; Waldner et al., 2015; Latchininsky et al., 2016).

With regard to L. migratoria migratoria, satellite images have been used to map spatiotemporal distribution of its preferred vegetation, the common reed Phragmites australis, in Central Asia (Sivanpillai et al., 2006; Latchininsky et al., 2007; Sivanpillai and Latchininsky, 2007, 2008; Propastin, 2012, 2013; Löw et al., 2013; Navratil and Wilps, 2013). Improved mapping of reed 
habitats has allowed for more targeted locust surveying and risk assessment (Latchininsky et al., 2016; Löw et al., 2016).

Satellite data have also been used for L. migratoria migratorioides habitat monitoring and post-outbreak damage assessment on vegetation in China (species given as L. migratoria manilensis; Ji et al., 2004; Ma et al., 2005; Zha et al., 2005; Tian et al., 2008). An attempt to apply Landsat data to estimate the risk of infestation by L. migratoria migratorioides in Madagascar was made by Franc et al. (2005; species given as L. migratoria capito).

Application of remote sensing data to other locust species is scarce. Franc et al. (2008) used SPOT satellite information to evaluate the impact of habitat fragmentation on population dynamics of $N$. septemfasciata in Madagascar. They showed that the SPOT images, combined with a digital elevation model, made it possible to identify three new migration pathways of $N$. septemfasciata, which were made possible by deforestation between 1986 and 2004. These pathways provided access to previously inaccessible oviposition zones and enabled locusts to breed, concentrate, and gregarise (Franc and Duranton, 2012; Latchininsky et al., 2016). Gornyy et al. (2011) and Tronin et al. (2014) attempted to estimate C. italicus outbreak potential in Eurasia using satellite information but encountered difficulties due to great habitat heterogeneity. Similarly, the patchiness and mosaic character of the habitats of D. maroccanus made it difficult to characterise its distribution in Central Asia based on satellite data (Smith, 2012).

Currently available satellites are unable to directly detect locust swarms or nymphal bands. An attempt by Kibasa (2006) to detect actual locust aggregations with the high-resolution SPOT 5 satellite data failed because it was not possible to distinguish locusts from the background soil.

A different remote sensing technique, so-called 'active remote sensing' in the form of Vertically Looking RADAR (VLR) was first used to monitor S. gregaria flights over the Sahara in the late 1960s (Roffey, 1969). VLR provided a tool for measuring aerial density, orientation, direction and speed of flight of solitarious locusts (Schaefer, 1969, 1976). VLR data could be a useful complement to routine locust surveys (Smith et al., 1993; Riley and Reynolds, 1997; Chapman et al., 2003). Although some specialists considered the use of VLR devices for monitoring locust swarm migrations impractical because of complex data analysis (Reynolds, 1988; Riley, 1989), this technique is currently used by the APLC in Australia for monitoring migrations of C. terminifera swarms (Drake et al., 2001; Deveson et al., 2005; Drake and Wang, 2013). 
A detailed history of remote sensing in locust monitoring and management is presented in Latchininsky and Sivanpillai (2010) and Latchininsky (2013). As soon as data from satellites became available for non-military purposes, remote sensing became a hope for locust specialists worldwide. Over the past three decades, satellite imagery has been increasingly applied to locust monitoring and has become a routine operational tool for two locust species, S. gregaria and $C$. terminifera (Cressman, 2013; Deveson, 2013). Based on satellite imagery, locust management teams target specific, high-risk gregarisation areas, which results in significantly reduced monitoring and management costs. This also contributes to a change in the paradigm of locust control from a curative to a preventive mode. Satellite data and associated GIS appear to be powerful instruments in the arsenal of locust managers, especially if accompanied by a thorough ground knowledge (Latchininsky and Sivanpillai, 2010). With advances in our understanding of the ecology of different locust species and the increased availability of satellites and processing software, remote sensing is gradually becoming an efficient tool for locust forecasting and management. Finally, the rapid development of remote sensing platforms, such as drones, is opening intriguing possibilities for various applications in locust research and management (see Section 8.2).

\subsection{CLIMATE CHANGE}

Climate change became obvious in the last few decades of the 20th century. One of the most powerful manifestations of climate change is global warming. Over the past hundred years the average temperature of the atmosphere near the Earth's surface has increased by $0.74^{\circ} \mathrm{C}$ (Houghton et al., 2001) and by the end of the $21^{\text {st }}$ century, the Earth's temperature is expected to rise between 1.8 and $4.6^{\circ} \mathrm{C}$ (IPPC, 2007).

Insects are ectotherms; their body temperature is strongly influenced by ambient air temperature, and their life cycles are usually short. Therefore, even small temperature variations can have a strong impact on population dynamics (Musolin and Saulich, 2012). Temperature increase, if it occurs within an optimal temperature range of a given species, is usually very beneficial for insects (Robinet and Rocques, 2010). In acridids, increased temperature can trigger a variety of responses, including a shift in spatial distribution, earlier hatching, and faster development. 
Despite the expected benefits of increased global temperatures for warm-climate species like locusts, warmer temperatures may also incur detrimental effects counteracting these benefits; for example, a lack of oviposition sites. Recent studies of historic Chinese records have suggested a correlation between climatic cycles and locust plagues, though the trend seems to vary with the spatiotemporal scale under scrutiny. Using the 1,000-year locust record compiled by Ma (1958), Stige et al. (2007) found that the mean abundance of L. migratoria migratorioides (named therein as L. migratoria manilensis) was negatively associated with temperature but positively associated with the frequencies of floods and droughts, both of which may have led to the creation of increased locust oviposition sites through either a waterbody receding, or the dampening of previously dry ground. The same authors (Zhang et al., 2009) used wavelet analysis to further analyse reported oscillations of locust population and climate, and suggested that periodic cooling events at 160- to 170-year intervals enhanced the likelihood of temperature-linked floods and droughts, the latter of which in particular led to a higher frequency of locust outbreaks. The implication of this work is that rising temperatures could reduce the likelihood of locust outbreaks in China, but the authors used locust data covering only the east of China and temperature data for the whole country, which could have led to regional inconsistencies. Indeed, analysis of the same long-term dataset by $\mathrm{Yu}$ et al. (2009) drew the opposite conclusion; using a general circulation model and wavelet analysis, the authors found a positive association between locust abundance and temperature at both inter-annual and inter-decadal scales. These contradictory results were partly reconciled by Tian et al. (2011), who reconstructed an even longer time series of locust outbreaks spanning 1,910 years (A.D. 2-1911) based on over 8,000 historic documents. Across an annual scale (A.D. 1512-1911) and decadal scale (A.D. 1000-1900) there were more locusts under dry and cold conditions, and when locusts were more abundant in the previous year or decade. Furthermore, using a 200-year moving window the authors showed that the correlation between locust abundance and precipitation was consistently negative between A.D. 500-1900, except for a few decades around A.D. 1200. By contrast, at a decadal resolution, the negative correlation between locust abundance and temperature per se was not as consistent, and was most convincing when the average temperature for the whole of China was used (and less strong at the regional and hemisphere scale). It is therefore clear that global or continent-wide trends may be too coarse a scale to allow accurate predictions, and that local and regional circumstances must be considered for effective locust forecasting and control. 


\subsubsection{Changes in distribution area}

Based on different climate simulation models, locust and grasshopper species of the Northern hemisphere could expand their distribution areas northwards. Olfert et al. (2011) predicted that $M$. sanguinipes is likely to inhabit more northerly Canadian latitudes than before. The distribution area of $\mathrm{Ca}$. italicus is already shifting to the north in Russia due to climate warming (Popova et al., 2016). Likewise, D. maroccanus has also undergone a northward expansion of its distribution limit in Turkmenistan and southern Kazakhstan (Latchininsky, 2017a). A corollary of northwards expansion, is colonisation of higher altitudes of mountains. Historically, D. maroccanus inhabits semi-deserts in foothills of the Mediterranean (sensu lato) region alongside spring ephemeral vegetation, which develops at altitudes between 400 and 1,000 m and includes Poa bulbosa, Alyssum desertorum, Carex pachystylis and Medicago falcata (Latchininsky and Launois-Luong, 1992). However, in recent years, climate warming has caused a gradual shift of vegetation belts to higher altitudes (Lenoir et al., 2008). As a result, in the $21^{\text {st }}$ century, D. maroccanus breeding habitats have moved to higher altitudes, with their vertical distribution limit shifting approximately $300 \mathrm{~m}$ higher than before. In Central Asia, D. maroccanus is now frequently found at altitudes of up to $1,800 \mathrm{~m}$ above sea level (Latchininsky et al., 2015).

\subsubsection{Earlier hatching and faster development}

An important result of these changes in phenology may be an increased number of annual generations (Nufio et al., 2010; Wu et al., 2012b). While most acridids from temperate Eurasia and North America are univoltine (having only one generation per year), with usually overwintering diapausing eggs (Latchininsky, 2017b), some species are recorded as having more than one annual generation. For example, late-season hatching of a second generation of the normally univoltine L. migratoria migratoria became frequent in Uzbekistan and southern Russia in the $21^{\text {st }}$ century (Stamo et al., 2013; Latchininsky et al., 2015). In North America, similar increases in annual generation number are reported for M. sanguinipes in Arizona (Jech, 2015). Even the embryonic development of grasshoppers, which takes place in the soil, occurs faster with higher ambient temperatures. In Alaska, grasshoppers Melanoplus spp. used to spend two winters 
as eggs in the soil, which meant a two-year long life cycle. However, a $3^{\circ} \mathrm{C}$ temperature increase in recent decades caused eggs to hatch after the first winter, resulting in a shift to a univoltine life cycle (Fielding and Defoliarte, 2010).

In summary, locust outbreaks are generally expected to become more frequent and severe under warming climatic conditions (Popova, 2014). Climate warming, which causes shifts in locust distribution, phenology and voltinism, is a serious challenge for both locust monitoring and management.

\subsection{LOCUST ECOLOGY CASE STUDY: THE CENTRAL AMERICAN LOCUST, SCHISTOCERCA PICEIFRONS}

The Central American Locust, Schistocerca piceifrons piceifrons, is an important pest species that has caused enormous damage to agriculture in Central America (Harvey, 1983; Barrientos Lozano et al., 1992; Contreras and Magaña, 2013). This species is widely distributed across the southern half of México to northern Costa Rica, with perpetual gregarious zones in Yucatán, and frequent outbreak areas in Veracruz, San Luis Potosí, Tamaulipas and Tabasco in México. Within México, this locust is known to impact 4.9 million hectares distributed throughout nine states causing enormous agricultural and economic damage, making it one of the most important pest species in México and Central America. This locust is a highly polyphagous species known to damage numerous crop plants, including maize, soybeans, wheat, sesame, cotton, sorghum, banana, sugarcane, and various fruits. Outbreaks of S. piceifrons are often associated with the dry season and high levels of starvation (Contreras and Galindo, 2013). Such outbreaks have been considered a possible explanation for the fall of the Mayan culture (Flores, 2011). This species has two generations per year, the first in summer and the second in winter. The first generation is shorter than the second one, which reaches maturity in the following spring (Hernandez-Zul et al., 2013).

S. piceifrons has two subspecies: $S$. piceifrons piceifrons from México to northern Costa Rica and S. piceifrons peruviana in Peru, Ecuador, Colombia, Venezuela, Panama, and Trinidad and Tobago (Harvey, 1983; Barrientos Lozano et al., 1992). S. piceifrons is a tropical locust and its geographic distribution coincides with areas with suitable vegetation and favorable weather conditions for breeding and development (Contreras and Magaña, 2013). Within these areas, there are permanent gregarious zones where the vegetation and environmental conditions promote 
feeding and breeding of S. piceifrons, which leads to outbreaks and subsequent transformation to the gregarious phase (Harvey, 1983; Barrientos Lozano et al., 1992).

\subsubsection{Vegetation}

Vegetation plays a major role in providing food and shelter for locusts. Studies on S. gregaria have shown that high population density is correlated with abundance of specific group of plants, like the dicotyledon Heliotropium arbainense and the monocotyledons Pennisetum typhodium, Aristida punsengens and Panicum turgidum (Woldewahid et al., 2004, 2007; Babah and Sword, 2004; Sword, 2010). In addition, there is also evidence that food distribution and nutritional quality can influence phase change (see Section 9). When the distribution of vegetation is patchy, locusts are more active, experience higher levels of crowding, and become more gregarious (Despland et al., 2000). The change in the threshold of gregarisation occurs according to the cover and status of the vegetation, in which low cover and dry vegetation lead to a low-density threshold of gregarisation, probably due to high probability of individuals mechanically stimulating each other

(Cissé et al., 2013). Unlike S. gregaria, S. piceifrons is attracted more generally by odour of any plants that can provide refuge and protection from natural enemies. For example, Pisonia aculeate is a large shrub that attracts $S$. piceifrons and promotes aggregation because it stays green during the dry season and can be patchily distributed (Poot-Pech et al., 2016).

\subsubsection{Land use}

Another important attribute of the gregarious zone is the pattern of land use. The original habitat of S. piceifrons was patchy grassland located in the same zones as dry tropical forest. However, a major problem in Latin America is the intensification of land use, and the principal habitat of $S$. piceifrons today has been created by human activity (Harvey, 1983). Between 2000 and 2010, forests were significantly reduced due to changes in agricultural or livestock activities, specifically in Yucatán State (the principal gregarisation area of México and Central America). In 1981 Yucatán had 394,236 ha of grassland, but in 2014 there were 579,634 ha. This increase of grassy habitats by over 185,000 ha within a 30-year time span served to increase the breeding zone of $S$. piceifrons (Poot-Pech, 2016). In Yucatán, the principal grass that provides suitable conditions for 
development of S. piceifrons is Panicum maximum (Poot-Pech et al., 2017). During the dry season, it is common practise among ranches in the gregarious zone to remove dried grasses by burning. While this practice promotes grass regrowth in the rainy season, it also causes aggregation of $S$. piceifrons by creating patches of bare soil, which are favoured areas for ovipositing (Poot-Pech, unpublished observations). In addition to creating favourable oviposition sites, excessive livestock grazing may also promote locust outbreaks by creating a nutritionally-optimal niche for locusts (e.g. Cease et al., 2012).

\subsubsection{Weather}

Characteristics of climate and soil contribute greatly to the fluctuation of population density of various insect species (Vinatier et al., 2011). In the case of locusts, temperature and soil moisture are highly correlated with egg laying and, in turn, increases in locust populations (Van der Werf et al., 2005; Xian-Lei et al., 2007; Hernández-Zul et al., 2013). Weather at a regional scale, particularly the distribution of precipitation, is also a critical factor that shapes the population density of locusts (de Wysiecki et al., 2011). Precipitation and the temperature averages are variable throughout the year in the breeding area. This variability can be seen from the data compiled for the Municipality of Buctzotz since 1961 to 2015 (Table 2), which is reported to be a major gregarious zone (Contreras and Magaña, 2013).

Precipitation is higher during the first generation than the second generation, and therefore locusts have access to sufficient food to complete their development as well as adequate soil moisture to oviposit for the second generation. In the first generation the temperature is the highest, and thus this generation of S. piceifrons is shorter than the second generation (Hernández-Zul et al., 2013). In the second generation, the period between January and May is the recession period, where population density is low (Barrientos Lozano et al., 1992). S. piceifrons start to mate when the precipitation increases in September (first generation) and May (second generation) (Harvey, 1983).

Understanding the influence of biotic and abiotic factors, such as vegetation and weather, on population dynamics of S. piceifrons is critical not only for predicting outbreaks, but also for effective field surveys (Symmons, 1992; Van der Werf et al., 2005; Sword, 2010). Further research 
in these areas will therefore offer some important tools to incorporate into management programs for this problematic species.

TABLE 2. Life history of S. piceifrons in Buctzotz, Mexico.

\begin{tabular}{|c|c|c|c|c|c|c|c|c|c|c|c|c|}
\hline \multirow{2}{*}{ Life stage } & \multicolumn{12}{|c|}{ Month } \\
\hline & Jan & Feb & Mar & Apr & May & Jun & Jul & Aug & Sep & Oct & Nov & Dec \\
\hline Nymph & & & & & & $\mathrm{x}$ & $\mathrm{x}$ & & & $\mathrm{x}$ & $\mathrm{x}$ & $\mathrm{x}$ \\
\hline Adult & $\mathrm{x}$ & $\mathrm{x}$ & $\mathrm{x}$ & $\mathrm{x}$ & $\mathrm{x}$ & & & $\mathrm{x}$ & $\mathrm{x}$ & & & $\mathrm{x}$ \\
\hline Swarming & $\mathrm{x}$ & $\mathrm{x}$ & & & & & & $\mathrm{x}$ & $\mathrm{x}$ & & & $\mathrm{x}$ \\
\hline Mating & & & & & $\mathrm{x}$ & $\mathrm{x}$ & & & $\mathrm{x}$ & & & \\
\hline Oviposition & & & & & $\mathrm{x}$ & $\mathrm{x}$ & & & $\mathrm{x}$ & & & \\
\hline Generation & \multicolumn{5}{|c|}{\begin{tabular}{|l|} 
Second generation \\
\end{tabular}} & \multicolumn{4}{|c|}{ First generation } & \multicolumn{3}{|c|}{ Second generation } \\
\hline Mean precipitation, $\mathrm{cm}$ & 45.4 & 36.4 & 24.8 & 26.3 & 82.3 & 160.5 & 169.9 & 185.1 & 211.0 & 125.4 & 49.4 & 42.4 \\
\hline Mean temperature, $\mathrm{C}$ & 22.5 & 23.5 & 25.7 & 27.8 & 28.7 & 28.5 & 28.0 & 28.0 & 27.7 & 26.0 & 24.2 & 22.8 \\
\hline
\end{tabular}

\section{Human - locust interactions}

Locusts have played a visible role throughout human history, with some of our earliest records noting outbreaks that had massive and destructive impacts on human populations and livelihoods. The early Chinese history classic, the Old Book of Tang, notes that in 785 CE: "The sky was shaded by the flying locusts. Hardly any vegetation and fur of animal remained after the place was visited by the plague of locusts. Roads were paved with starved people. Those survived steamed the locusts to eat as food." (Liu, 1975). Similarly, the book of Exodus (10:15 RSV) describes a particularly brutal locust plague: "They covered the face of the whole land so that the land was darkened, and they ate all the plants in the land and all the fruit of the trees which the hail had left; not a green thing remained, neither tree nor plant of the field, through all the land of Egypt." These early records reveal the long-standing connections between humans and locusts. While these are likely records of some of the most extreme events, they also reveal locusts to have been a common occurrence in human society throughout much of our history.

Today, of course, locusts still play a role in many communities, predominantly through impacts on agriculture. In lesser developed regions, where human-locust relationships are still 
tightly linked, we often find the populations that are most vulnerable to locust outbreaks. West Africa, for example, is highly susceptible to locust outbreaks or even persistent low-level crop degradation, and the impacts can be devastating for many rural dwellers (Brader et al., 2006). These impacts can have ripple effects in other areas of life. For example, outbreaks in Mali have been linked with decreased educational attainment for the children of affected families (de Vreyer et al., 2015). Low levels of capital assets in these areas make pre-emptive biological control difficult, and international agencies typically provide support only when outbreaks have reached "emergency" status. Therefore, farmers in these kinds of situations can only react to locust problems when they occur. In industrialised regions, prevention and planning is often incorporated into agricultural management strategies, primarily through the use of insecticides to control locusts when even moderate densities begin to appear (Hunter et al., 2004). Still, prevention and control of locusts is far from solved, and many mysteries and challenges remain. Here we briefly review some perspectives from the social sciences, particularly from the viewpoint of agricultural and environmental economics, that may lend some insight into the nature of what might and might not work for better managing future outbreaks.

In general, we want to identify management levers that would allow individuals or policy makers to affect the likelihood of locust outbreaks occurring. The impact of locusts is greatest at the level of the farm; however, damage is heterogeneous and widespread across landscapes. The result is that the scope of intervention is diffuse and shared among many stakeholders. The mismatch of these scales creates an obstacle to sustainable locust management (Péloquin, 2014; Lockwood et al., 2001). Locusts are a continental-level problem that requires coordinated responses across boundaries by individuals and governments. With a better understanding of the connections between land management decisions and locusts, action at the level of farms or small communities may be the best option for decreasing the probability of outbreaks. For example, introduction of European livestock and agriculture to Australia over the past century may have contributed to present-day C. terminifera swarms (Deveson, 2012). Similarly, in Yucatán State, the main gregarisation area of $S$. piceifrons in México and Central America, deforestation due to agriculture led to a 50\% increase in grassy breeding areas (Poot-Pech, 2016; see Section 10.3). This pattern for S. piceifrons was exacerbated by expansions of the invasive grass Panicum maximum, which creates favourable conditions for S. piceifrons (Poot-Pech et al., 2017). In Inner Mongolia, China, Cease et al. (2012) found that heavy livestock grazing promotes O. asiaticus 
outbreaks by lowering plant nitrogen content. Continuous high levels of livestock grazing lead to loss of topsoil and soil nitrogen (Giese et al., 2013). Reduced soil fertility decreases nitrogen content of grass leaves, which results in low protein, high carbohydrate leaves. In contrast to the common conception of herbivores being nitrogen-limited, O. asiaticus prefers and grows best on low protein, high carbohydrate diets; high protein diets decrease locust survivorship (Cease et al., 2012). Furthermore, these low protein, high carbohydrate diets, in conjunction with high population density, promote development of migratory characteristics (Cease et al., 2017), presumably promoting increased capacity for long-distance migration. While these examples illustrate mechanisms by which individual farm managers might keep locusts at bay, they may not have the resources, knowledge, or incentive to maintain preventative locust management. Farm managers need to consider many potential outcomes and shocks, such as drought or market fluctuations, in addition to locust plagues. Therefore, it is important to approach sustainable locust management from a systems perspective (Figure 2).

To investigate the potential for sustainable farm-level locust management, we begin by looking at the household and farmers' decision-making. Agricultural production requires the farmer to manage productive assets considering a short-run (i.e., $<12$ months) and long-run (i.e., $>12$ months) planning horizon jointly (Kay et al., 2012). Ideally, short-run decisions help the farm manager reach their long-run goals. Long-run goals may include increasing the number of animals in their grazing herd, increasing profitability, having consistent profit over time, or more generally increasing productive assets. Productive assets are agricultural items, such as livestock or land, which are managed to create revenue flows to the farm manager over time. While these assets contribute to the success of the agricultural operation, in developing countries they also provide the decision maker with a form of wealth accumulation or a "savings account" to protect against risk, such as locust plagues. Knowing that these assets also provide a form of insurance for the farmer suggests that they will be managed in a way that emphasises long-term sustainability. The objectives and action plans for short and long-run farm level decisions vary by farm manager. Short-run decisions are often focussed on solving a financial hardship and can overlook environmental sustainability, regulations, or ecosystem effects. For example, a farmer may sell livestock or overgraze a pasture to feed the animals to a larger size to pay outstanding debt. Within this decision, they are not concerned about future output, but only what needs to be done today to survive to the next year. When farm managers make long-run decisions they consider how the 
decisions they make today will affect their operation in the future. While considering these factors, farm managers can enhance the long-run productivity of their farm operation by including information on the long-term effects of stocking decisions on the quality of their land and their overall farm output. Considering the complexity of the decision-making process it is important to approach farm management with a whole systems perspective. For example, in China overgrazing may increase farm-level output (e.g. more sheep in the short term), but degrades soil quality and may result in ecosystem externalities, such as a locust outbreak in the long-term (Cease et al., 2012).

Even if farmers effectively manage locusts on their property, the remaining key problem is the familiar tragedy of the commons: if one farmer invests in preventative locust measures on their lands, they may not reap all (or even most) of the benefits of that investment. Most likely, their neighbours would benefit greatly from their actions. If multiple farm managers begin to make short-run decisions disregarding the long-term effects, ecosystem externalities such as locust outbreaks can occur more consistently. Moreover, locusts are migratory and connect not only neighbouring farms but also distant landscapes. Therefore, when thinking of locust management broadly, local farmers may be agents that can help in an overall strategy, but the management structure must fit the spatial extent of the ecological problem (Young, 2002). In the case of locusts, the scale can be continent-wide.

Effective, economically efficient, and sustainable management of locust plague risk requires an interdisciplinary approach in ways that parallel other integrations of natural and social sciences. On one hand, attempts to model locust management strategies that focus only on the financial cost/benefit do not reflect true economic cost. True economic value must incorporate trade-offs faced by individuals, making it incorrect to equate the economic cost of a policy with the accounting cost of an action (Bockstael et al., 2000). On the other hand, for modelling efforts that omit economic cost and instead focussed on solely on the natural science, ignoring the role and responses of humans means omitting an integral subsystem of the social-ecological system, (Ostrom, 2009). For locust social-ecological systems, these feedbacks include (but are not limited to) interactions between plant quality and locust and livestock growth rates, decisions made by farmers and ranchers on how to use land and the choices of how to control outbreaks (Cease et al., 2012, 2015; Ali, 2008; Lecoq, 2005). Modelling results of social-ecological systems are difficult 
to predict and often nonlinear, and depend on the choices made about the subsystems being modelled (Ostrom, 2009).

Bio-economic modelling, which links trade-offs resulting from complex ecosystem dynamics with the consequences of policy attempting to mitigate risk such as locust plagues, may be one field that can yield particular insight (Crocker and Tschirhart, 1992). Bio-economic modelling can be used if we can define the costs, benefits, and management mechanisms that are associated with the different aspects of the natural system. General equilibrium externalities occur when there are impacts and feedbacks between various sectors and species (Finnoff and Tschirhart, 2008; Carbone and Smith, 2008). Since locusts are migratory, locust swarms may arrive and impact areas that are well managed by farmers and policymakers. Understanding the relationship between locust control and the timing and type of locust policy intervention suggests that social science could contribute to management responses by integrating human preferences for risk within locust population models.

Excluding humans from ecosystem models means that model parameters and results are accurate only within the context of existing human institutions. Humans respond to incentives, and ecosystems are subject to these changes in human behaviour. Incorporating potential risk mitigation strategies, such as preventing a plague, controlling an existing swarm, or adapting to a plague by limiting the risk to humans of adverse impacts, is important to capture the feedback loops and interactions across the ecosystem (Ehrlich and Becker, 1972; Finnoff et al., 2010). When considering the cost or benefits of any of these policies, their impacts must be compared against each other and in conjunction with each other (Shogren and Kane, 2000). When human preferences, such as negative farmer perceptions of the environmental and health costs of insecticide spraying, are included, alternatives such as insurance may be preferable (Ali, 2008). The benefits of each policy are going to be connected to each other - if nothing is at risk because farmers have perfectly adapted to locust outbreaks (or any adverse event), there is no benefit to preventing an outbreak from occurring. For this reason, temporal and spatial scales, in addition to the biophysical and social contexts, need to be defined and integrated to find effective, economically efficient, and sustainable solutions to locust plagues (Bockstael et al., 2000; Cease et al., 2015; Péloquin, 2014; Lockwood et al., 2001; Ostrom, 2009; Figure 2). 


\section{Concluding remarks}

The last ten years have seen dramatic progress and challenges in our understanding of locust phase change. The opportunities provided by advances in molecular biology are now being applied to locust research, and the sequencing of the L. migratoria genome (Wang et al., 2014) is a significant milestone. The size and complexity of the genome, together with the relatively low level of annotation and/or detectable sequence identity with those of other organisms, in some ways serves to underline the daunting task understanding the molecular basis of phase change in its entirety. Nevertheless, techniques such as RNAi knockdown have become standard tools in experimentally manipulating putative mechanisms of phase change. The new and powerful CRISPR-Cas9 (clustered regularly interspaced short palindromic repeats) technique is a means to edit the genome and induce permanent changes in gene expression. At the time of writing only a single paper has reported this technique in locusts (Li et al., 2016), which used it to successfully delete an odorant receptor co-receptor (Orco) in L. migratoria, but it is to be expected that use of this technique will greatly increase in the future. The demonstration of the existence of widespread DNA methylation in locusts has spurred interest in the epigenetic nature of phase change and the transmission of phase characteristics to offspring. Determining its exact nature will be a substantial research focus of the next ten years.

Biogenic amine signalling and its role in at least the early stages of behavioural phase transition have remained a subject of interest, but the picture has become considerably more complex as more information becomes available. All the major biogenic amines have now been implicated in the process of gregarisation and / or solitarisation. In part this probably reflects the independent evolution of density-dependent behavioural change in S. gregaria and L. migratoria, two distantly related species, which have co-opted somewhat different widespread and evolutionarily conserved mechanisms of neuronal plasticity to produce similar phenotypic outcomes. A challenge is now to unravel this complex pattern and match the roles of specific biogenic amines to particular aspects of behavioural change. This process will be assisted by recent progress in the behavioural analysis of locusts; automated tracking facilitates a greater throughput and helps generate more data more quickly, whilst new quantitative methods and statistical models will help extract more information from the data and help distinguish behavioural phase change from other kinds of behavioural response. 
The interest in comparative analyses between different locust species, and between locusts and 'near locusts' that are either closely related to locusts or show some of the aspects of plasticity associated with full phase polyphenism, is to be welcomed. Such comparative analyses promise to increase our understanding of how phase polyphenism has evolved, and may help resolve some of the difficulties of how different physiological mechanisms occur in different species. As research spreads beyond the two canonical locusts, S. gregaria and L. migratoria, to other species that in their own locales may be just as important or damaging, a larger framework unifying and relating our understanding of these different species becomes essential.

Locusts have remained and will continue to be an inspiration and model species for those interested in the emergent behaviour of collective movement. The extension of experimental studies beyond the laboratory to larger scale field settings is an important development and is poised to yield important insights into the structure of locust swarms in particular, and whole field of collective motion studies in general.

There has been a renewed interest in how environmental factors affect phase change and the development of outbreaks or larger scale swarms. Most laboratory studies have focussed on simplified conditions to try and understand how locust-locust interactions initiate the process of phase change, but in natural environments other factors greatly influence the likelihood of locusts encountering each other and may even directly influence the mechanisms of phase change. Nutritional status is emerging as an important factor in phase change itself and the propagation of locust outbreaks. Locust outbreaks and plagues, and the economic damage they inflict, exist in a wider context of human land use and management. The demonstration of the role that human activities may unwittingly play in creating conditions that facilitate gregarisation and ultimately swarm formation has been an important breakthrough.

Locust phase change research is motivated by several reasons; as a model for phenotypic plasticity generally, or neuronal and behavioural plasticity in particular, or as an exemplar of collective movement, but all of this stems from the status of locusts as major and occasionally catastrophic pest species that warrant continual monitoring and control. The inclusion of a section that analyses locusts within social-ecological systems, including the economics of locust outbreaks, is unusual in a review of this kind, but serves to underline the wider human context in which locust research occurs. The locust phase change research community is small, but the range of research interests covered within it, from molecules to management, is all encompassing as 
presented in this review. Major research opportunities and insights in the future will depend on crossing disciplinary boundaries and fusing different tiers of expertise to create new and deeper insights into locust phase transformation and its relationship to humans, agriculture, and the environment. To this end, Arizona State University is supporting development of the Global Locust Initiative (GLI). This Initiative's purpose is to assist universities, government and nongovernment agencies, and agricultural communities to forge new partnerships that seek to better

integrate locust research and management. GLI is based on three pillars: advancing fundamental research, creating and maintaining a global network, and developing local solutions to the global challenge of locust plagues. GLI will develop and maintain a global database of interested people and organisations, along with relevant documents and data such as research methods and locust outbreak records. This network is intended to remove barriers for new people and organisations to work on locusts, and for current researchers and managers working across sectors and geographical regions to collaborate more easily. GLI will support workshops and the development of funding proposals for interdisciplinary teams advancing fundamental research, and for cross-sectoral teams fusing fundamental and applied approaches to develop new solutions to the ancient challenge of locust plagues.

\section{Acknowledgements}

We would like to thank The Entomological Society of America for hosting our two symposia at ICE 2016, the $25^{\text {th }}$ International Congress of Entomology in Orlando, Florida. We would also like to gratefully thank the following funding bodies for their support:

DAC, JVdB, RV and HV were supported by the Research Foundations of Flanders (FWO) and KU Leuven (GOA/11/02 and C14/15/050). RV was also supported by an FWO PhD Fellowship; DAC and HV are supported by FWO Postdoctoral Fellowships.

AJC, KB, JCH and BR were supported by the U.S. National Science Foundation (\#1313693). AJC was also supported by (CHE-1313958), and BR was also funded by Fonds de Recherche du Québec - Société et culture (2015-NP-182013). 
AL was partly funded by a Wyoming NASA Space Grant.

AA was partially supported by grant no. 891-0277-13 from Israel's Ministry of Agriculture.

JB was funded by the Australian Research Council Future Fellowship, Discovery Projects and Linkage Projects programs.

RDK, TM and SRO were supported by research grant BB/L02389X/1 (to SRO and TM) from the Biotechnology and Biological Sciences Research Council, UK, and a Wellcome Trust ISSF award from the University of Leicester.

JMS was supported by a MIBTP Studentship from the Biotechnology and Biological Sciences Research Council, UK.

BF and HS were supported by the U.S. National Science Foundation (IOS-1253493).

MAP-P was supported by Yucatán Plant Health (CESVY) and the National Service of Sanitary and Agro-Alimentary Health (SENASICA), México.

GAS was supported by USDA-NIFA, Texas A\&M University AgriLife Research, and the Charles R. Parencia Endowment.

\section{References}

Alessi, A.M., O’Connor, V., Aonuma H., Newland, P.L., 2014. Dopaminergic modulation of phase reversal in desert locusts. Front. Behav. Neurosci. 8, 1-15.

Ali, H.A., Mohammed, B., 2008. Cost benefit analysis of desert locusts' control: a multicountry perspective. Economic Research Forum Working Papers. No. 0801.

Altizer, S., Bartel, R., Han, B.A. 2011. Animal migration and infectious disease risk. Science 331, 296-302. 
Amichay, G., Ariel, G., Ayali, A., 2016. The effect of changing topography on the coordinated marching of locust nymphs. PeerJ 4, e2742.

Andiatsirevombola, J.M., Ge, J., Nyirenda, T.M., Raholijaona, 2016. Predation impact of Cattle Egret (Bubulcus ibis) on Migratory Locust (Locusta migratoria capito) and Red Locust (Nomadacris septemfasciata) in south and southwest regions of Madagascar. Appl. Ecol. Env. Res. 14, 297-308.

Andretic, R., Van Swinderen, B., Greenspan, R.J., 2005. Dopaminergic modulation of arousal in Drosophila. Curr. Biol. 15, 1165-1175.

Angers, B., Castonguay, E., Massicotte, R., 2010. Environmentally induced phenotypes and DNA methylation: how to deal with unpredictable conditions until the next generation and after. Mol. Ecol. 19, 1283-1295.

Anstey, M.L., Rogers, S.M., Ott, S.R., Burrows, M., Simpson, S.J., 2009. Serotonin mediates behavioral gregarization underlying swarm formation in Desert Locusts. Science 323, 627-630.

Applebaum, S. W., Heifetz, Y. 1999. Density-dependent physiological phase in insects. Annu. Rev. Entomol. 44, 317-341.

Ariel, G., Ophir, Y., Levi, S., Ben-Jacob, E., Ayali, A., 2014. Individuals' intermittent motion is instrumental to the formation and maintenance of swarms of marching locust nymphs. PLoS ONE 9, e101636.

Ariel, G., Ayali A., 2015. Locust collective motion and its modeling. PLoS Comput. Biol. 11, e1004522.

Ashall, C., Ellis, P.E., 1962. Studies on numbers and mortality in field populations of the Desert Locust (Schistocerca gregaria Forskål). Anti-Locust Bull. 38, 1-59.

Babah, M.A.O., Sword, G.A., 2004. Linking locust gregarization to local resource distribution patterns across a large spatial scale. Environ. Entomol. 33, 1577-1583.

Badisco, L., Huybrechts, J., Simonet, G., Verlinden, H., Marchal, E., Huybrechts, R., Schoofs, L., De Loof, A., Vanden Broeck, J., 2011a. Transcriptome analysis of the desert locust central nervous system: production and annotation of a Schistocerca gregaria EST database. PLoS ONE 6, e17274.

Badisco, L., Ott, S.R., Rogers, S.M., Matheson, T., Knapen, D., Vergauwen, L., Verlinden, H., Marchal, E., Sheehy, M.R.J., Burrows, M., Vanden Broeck, J., 2011b. Microarray-based transcriptomic analysis of differences between long-term gregarious and solitarious desert locusts. PLoS ONE 6, e28110.

Balfanz, S., Strünker, T., Frings, S., Baumann, A., 2005. A family of octopamine [corrected] receptors that specifically induce cyclic AMP production or $\mathrm{Ca} 2+$ release in Drosophila melanogaster. J. Neurochem. 93, 440-451. 
Barrientos Lozano, L., 2011. Dinámica Poblacional, Biología y Ecología de la Langosta Suramericana (Schistocerca cancellata, Serville). Instituto Tecnológico de Ciudad Victoria, Tamaulipas, México. Pp. 93-135.

Barrientos Lozano, L., Astacio, C.O., Alvarez, B.F., Poot, M.O., 1992. Manual técnico sobre la langosta voladora (Schistocerca piceifrons piceifrons Walker 1870) y otros acridoideos de Centroamérica y Sureste de México. FAO-OIRSA, El Salvador.

Bayliss, A., Roselli, G., Evans, P.D., 2013. A comparison of the signalling properties of two tyramine receptors from Drosophila. J. Neurochem. 125, 37-48.

Bazazi, S., Bartumeus, F., Hale, J.J., Couzin, I.D., 2012. Intermittent motion in desert locusts: behavioural complexity in simple environments. PLoS Comput. Biol. 8, e1002498.

Bazazi, S., Buhl, J., Hale, J.J., Anstey, M.L., Sword, G.A., Simpson, S.J., Couzin I.D., 2008. Collective motion and cannibalism in locust migratory bands. Curr. Biol. 18, 735-739.

Bazazi, S., Romanczuk, P., Thomas, S., Schimansky-Geier, L., Hale, J.J., Miller, G.A., Sword, G.A., Simpson, S.J., Couzin, I.D., 2011. Nutritional state and collective motion: from individuals to mass migration. Proc. Roy. Soc. B Biol. Sci. 278, 356-363.

Beaulieu, J.M., Gainetdinov, R.R., 2011. The physiology, signaling, and pharmacology of dopamine receptors. Pharmacol. Rev. 63, 182-217.

Beggs, K.T., Hamilton, I.S., Kurshan, P.T., Mustard, J.A., Mercer, A.R., 2005. Characterization of a D2-like dopamine receptor (AmDOP3) in honey bee, Apis mellifera. Insect Biochem. Mol. Biol. 35, 873-882.

Beggs, K.T., Mercer, A.R., 2009. Dopamine receptor activation by honey bee queen pheromone. Curr. Biol. 19, 1206-1209.

Beggs, K.T., Tyndall, J.D., Mercer, A.R., 2011. Honey bee dopamine and octopamine receptors linked to intracellular calcium signaling have a close phylogenetic and pharmacological relationship. PLoS ONE 6, e26809.

Belayneh, Y.T., 2005. Acridid pest management in the developing world: a challenge to the rural population, a dilemma to the international community. J. Orthoptera Res. 14, 187-195.

Bellés, X., 2010. Beyond Drosophila: RNAi in vivo and functional genomics in insects. Annu. Rev. Entomol. 55, 111-128.

Bernays, E.A., Lee, J., 1988. Food aversion learning in the polyphagous grasshopper Schistocerca americana. Physiol. Entomol. 13, 131-137.

Berthier, K., Chapuis, M.-P., Simpson, S.J., Ferenz, H.-J., Habib Kane, C.M., Kang, L., Lange, A., Ott, S.R., Ould Babah, M.A., Rodenburg, K.W., Rogers, S.M., Torto, B., Vanden Broeck, J., Van Loon, J.J.A., Sword, G.A., 2010. Laboratory populations as a resource for understanding the relationship between genotypes and phenotypes: a global case study in locusts. Adv. Insect Physiol. 39, 1-37. 
Bewick, A.J., Vogel, K.J., Moore A.J., Schmitz R.J., 2016. Evolution of DNA methylation across insects. Mol. Biol. Evol. 34, 654-665.

Bischof, L.J., Enan, E.E., 2004. Cloning, expression and functional analysis of an octopamine receptor from Periplaneta americana. Insect Biochem. Mol. Biol. 34, 511-521.

Bischoff, J.F., Rehner, S.A., Humber, R.A., 2009. A multilocus phylogeny of the Metarhizium anisopliae lineage. Mycologia 101, 512-530.

Blackburn, L.M., Ott, S.R., Matheson, T., Burrows, M., Rogers, S.M., 2010. Motor neurone responses during a postural reflex in solitarious and gregarious desert locusts. J. Insect Physiol. $56,902-910$.

Blanchet, E., Lecoq, M., Sword, G.A., Berthier, K., Pages, C., Billot, C., Rivallan, R., Foucart, A., Vassal, J.-M., Risterucci, A., 2011. A comparative analysis of fine-scale genetic structure in three closely related syntopic species of the grasshopper genus Calliptamus. Can. J. Zool. 90, 31-41.

Blenau, W., Balfanz, S., Baumann, A., 2000. Amtyr1: characterization of a gene from honeybee (Apis mellifera) brain encoding a functional tyramine receptor. J. Neurochem. 74, 900-908.

Blenau, W., Baumann, A., 2001. Molecular and pharmacological properties of insect biogenic amine receptors: lessons from Drosophila melanogaster and Apis mellifera. Arch. Insect Biochem. Physiol. 48, 13-38.

Blenau, W., Erber, J., 1998. Behavioural pharmacology of dopamine, serotonin and putative aminergic ligands in the mushroom bodies of the honeybee (Apis mellifera). Behav. Brain Res. 96, 115-124.

Blenau, W., Erber, J., Baumann, A., 1998. Characterization of a dopamine D1 receptor from Apis mellifera: cloning, functional expression, pharmacology, and mRNA localization in the brain. J. Neurochem. 70, 15-23.

Bockstael, N.E., Freeman, A.M., Kopp, R.J., Portney, P.R., Smith, V.K., 2000. On measuring economic values for nature. Environ. Sci. Technol. 34, 1384-1389.

Boerjan, B., Sas, F., Ernst, U.R., Tobback, J., Lemière, F., Vandegehuchte, M.B., Janssen, C.R., Badisco, L., Marchal, E., Verlinden, H., Schoofs, L., De Loof, A., 2011. Locust phase polyphenism: does epigenetic precede endocrine regulation? Gen. Comp. Endocrinol. 173, 120128.

Bouaïchi, A., Roessingh, P., Simpson, S.J., 1995. An analysis of the behavioural effects of crowding and re-isolation on solitary-reared adult desert locusts (Schistocerca gregaria) and their offspring. Physiol. Entomol. 20, 199-208.

Bouaïchi, A., Simpson, S.J., Roessingh, P., 1996. The influence of environmental microstructure on the behavioural phase state and distribution of the desert locust Schistocerca gregaria. Physiol. Entomol. 21, 247-256. 
Brader, L., Djibo, H., Faye, F., Ghaout, S., Lazar, M., Luzietoso, P., Babah, M.O., 2006. Towards a more effective response to desert locusts and their impacts on food security, livelihoods and poverty: Multilateral evaluation of the 2003-05 desert locust campaign. FAO, Rome.

Bradley, S.P., Chapman, P.D., Lizbinski, K.M., Daly, K.C., Dacks, A.M., 2016. A flight sensorymotor to olfactory processing circuit in the moth Manduca sexta. Front. Neural Circuits 10, 5.

Bryceson, K.P., 1984. Discrimination of small areas of vegetation growth after localised rain in semiarid environments using Landsat. Proc. 3rd Australian Remote Sensing Conference, 541-550.

Bryceson, K.P., 1989. The use of Landsat MSS data to determine the distribution of locust eggbeds in the Riverina region of New South Wales, Australia. Int. J. Remote Sens. 10, 1749-1762.

Bryceson, K.P., 1990. Digitally Processed Satellite Data as a tool in detecting potential Australian Plague locust outbreak areas. J. Environ. Manage. 30, 191-207.

Bryceson, K.P., 1991. Likely locust infestation areas in western New South Wales, Australia, located by satellite. Geocarto Int. 6, 21-37.

Bryceson, K.P., Wright, D.E., 1986. An analysis of the 1984 locust plague in Australia using multitemporal Landsat multispectral data and a simulation model of locust development. Agr. Ecosyst. Environ. 16, 87-102.

Buhl, E., Schildberger, K., Stevenson, P.A., 2008. A muscarinic cholinergic mechanism underlies activation of the central pattern generator for locust flight. J. Exp. Biol. 211, 2346-2357.

Buhl, J., Rogers, S.M., 2016. Mechanisms underpinning aggregation and collective movement by insect groups. Curr. Opin. Insect Sci. 15, 125-130.

Buhl, J., Sumpter, D.J.T., Couzin, I.D., Hale, J.J., Despland, E., Miller, E.R., Simpson, S.J., 2006. From disorder to order in marching locusts. Science 312, 1402-1406.

Buhl, J., Sword, G.A., Simpson, S.J., 2012. Using field data to test locust migratory band collective movement models. Interface Focus 2, 757-763.

Buhl, J., Sword, G.A., Clissold, F.J., Simpson, S.J., 2011. Group structure in locust migratory bands. Behav. Ecol. Sociobiol. 65, 265-273.

Burgess, S.C., Baskett, M.L., Grosberg, R.K., Morgan, S.G., Strathmann, R.R., 2015. When is dispersal for dispersal? Unifying marine and terrestrial perspectives. Biol. Rev. 91, 867-882.

Burrows, M., Rogers, S.M., Ott, S.R., 2011. Epigenetic remodelling of brain, body and behaviour during phase change in locusts. Neural Syst. Circuits 1, 11.

Butler, C., Innes, J., 1936. A comparison of the rate of metabolic activity in the solitary and migratory phases of Locusta migratoria. Proc. Roy. Soc. B Biol. Sci., 119, 296-304.

Callier, S., Snapyan, M., Le Crom, S., Prou, D., Vincent, J.D., Vernier, P., 2003. Evolution and cell biology of dopamine receptors in vertebrates. Biol. Cell 95, 489-502. 
Camacho, J.P.M., Ruiz-Ruano, F.J., Martín-Blázquez, R., López-León, M.D., Cabrero, J., Lorite, P., Cabral-de-Mello, D.C., Bakkali, M., 2015. A step to the gigantic genome of the desert locust: chromosome sizes and repeated DNAs. Chromosoma 124, 263-275.

Camazine, S., Deneubourg, J.-L., Franks, N.R., Sneyd, J., Theraulaz, G., Bonabeau, E., 2003. Selforganization in biological systems. Princeton University Press, New Jersey.

Carbone, J.C., Kerry Smith, V., 2008. Evaluating policy interventions with general equilibrium externalities. J. Public Econ. 92, 1254-1274.

Cardinaud, B., Gilbert, J.M., Liu, F., Sugamori, K.S., Vincent, J.D., Niznik, H.B., Vernier, P., 1998. Evolution and origin of the diversity of dopamine receptors in vertebrates. Adv. Pharmacol. 42, 936-940.

Cazzamali, G., Klaerke, D.A., Grimmelikhuijzen, C.J.P., 2005. A new family of insect tyramine receptors. Biochem. Biophys. Res. Comm. 338, 1189-1196.

Cease, A.J., Elser, J.J., Fenichel, E.P., Hadrich, J.C., Harrison, J.F., Robinson, B.E., 2015. Living with locusts: connecting soil nitrogen, locust outbreaks, livelihoods, and livestock markets. BioScience 65, 551-558.

Cease, A.J., Elser, J.J., Ford, C.F., Hao, S., Kang, L., Harrison, J.F., 2012. Heavy livestock grazing promotes locust outbreaks by lowering plant nitrogen content. Science 335, 467-469.

Cease, A.J., Hao, S., Kang, L., Elser, J.J., Harrison, J.F., 2010. Are color or high rearing density related to migratory polyphenism in the band-winged grasshopper, Oedaleus asiaticus? J. Insect Physiol. 56, 926-936.

Cease, A.J., Harrison, J.F., Hao, S., Niren, D.C., Zhang, G., Kang, L., Elser, J.J. 2017. Nutritional imbalance suppresses migratory phenotypes of the Mongolian locust (Oedaleus asiaticus). Roy. Soc. Open Sci. 4, 161039.

Chapman, J.W., Reynolds, D.R., Smith, A.D., 2003. Vertical-looking radar: A new tool for monitoring high-altitude insect migration. BioScience 53, 503-511.

Chapman, R.F., 1954. Responses of Locusta migratoria migratorioides (R. \& F.) to light in the laboratory. British J. Anim. Behav. 2, 146-152.

Chapuis, M.-P., Bazelet, C.S., Blondin, L., Foucart, A., Vitalis, R., Samways, M.J., 2016. Subspecific taxonomy of the desert locust, Schistocerca gregaria (Orthoptera: Acrididae), based on molecular and morphological characters. Syst. Entomol. 41, 516-530.

Chapuis, M.-P., Foucart, A., Plantamp, C., Blondin, L., Leménage, N., Benoit, L., Gay, P.-E., Bazelet, C.S., 2017. Genetic and morphological variation in non-polyphenic southern African populations of the desert locust. Afr. Entomol. 25, 13-23.

Chapuis, M.-P., Loiseau, A., Michalakis, Y., Lecoq, M., Franc, A., Estoup, A., 2009. Outbreaks, gene flow and effective population size in the migratory locust, Locusta migratoria: a regional scale comparative survey. Mol. Ecol. 18, 792-800. 
Chapuis, M.-P., Plantamp, C., Blondin, L., Pages, C., Vassal, J.-M., Lecoq, M., 2014. Demographic processes shaping genetic variation of the solitarious phase of the desert locust. Mol. Ecol. 23, 1749-1763.

Chapuis, M.-P., Popple, J.-A.M., Berthier, K., Simpson, S.J., Deveson, E., Spurgin, P., Steinbauer, M.J., Sword, G.A., 2011. Challenges to assessing connectivity between massive populations of the Australian plague locust. Proc. Roy. Soc. B Biol. Sci. 278, 3152-3160.

Chen, H., Scherkenbeck, J., Zdobinsky, T., Antonicek, H., 2010a. Fluorescent analogues of the insect neuropeptide helicokinin I: synthesis, photophysical characterization and biological activity. Protein Pept. Lett. 17, 431-436.

Chen, S., Yang, P., Jiang, F., Wei, Y., Ma, Z., Kang, L., 2010b. De novo analysis of transcriptome dynamics in the Migratory Locust during the development of phase traits. PLoS ONE 5, e15633.

Cherlet, M., RAMSES Reconnaissance and Management System of the Environment of Schistocerca (Desert Locust). Programme outline on future requirements in remote sensing and modeling and their integration using GIS to improve Desert Locust forecasting, Project Report GCP/INT/439/BEL, 1993, FAO, Rome.

Cherlet, M., Di Gregorio, A., 1993. Calibration and integrated modelling of remote sensing data for Desert locust habitat monitoring. RSC Series 64, FAO, Rome.

Cigliano, M.M., Braun, H., Eades, D.C., Otte, D., 2017. Orthoptera Species File. Version 5.0/5.0. [03/10/2017]. <http://Orthoptera.SpeciesFile.org>.

Cissé, S., Ghaout, S., Mazih, A., Ebbe, B., Ould, M.A., Benahi, A.S., Piou, C., 2013. Effect of vegetation on density thresholds of adult desert locust gregarization from survey data in Mauritania. Entomol. Exp. Appl. 149, 159-165.

Cissé, S., Ghaout, S., Mazih, A., Ebbe, O.B., Abdallahi, M., Piou, C., 2015. Estimation of density threshold of gregarization of desert locust hoppers from field sampling in Mauritania. Entomol. Exp. Appl. 156, 136-148.

Cissé, S., Ghaout, S., Babah Ebbe, M.A., Kamara, S., Piou, C., 2016. Field verification of the prediction model on desert locust adult phase status from density and vegetation. J. Insect Sci., 16, 74.

Clark, L.R., 1949. Behaviour of swarm hoppers of the Australian plague locust (Chortoicetes terminifera Walker). CSIRO Bull. 245, 1-27.

Clobert, J., Galliard, L., Cote, J., Meylan, S., Massot, M., 2009. Informed dispersal, heterogeneity in animal dispersal syndromes and the dynamics of spatially structured populations. Ecol. Lett. 12, 197-209.

Cloudsley-Thompson, J.L., 1977. Diurnal rhythms of locomotory activity in isolated desert locusts (Schistocerca gregaria (Forsk.). J. Interdisc. Cycle Res. 8, 27-36. 
Colas, J.F., Launay, J.M., Kellermann, O., Rosay, P., Maroteaux, L., 1995. Drosophila 5-HT2 serotonin receptor: coexpression with fushi-tarazu during segmentation. Proc. Natl. Acad. Sci. U.S.A. 92, 5441-5445.

Collett, T.S., 1978. Peering - a locust behaviour pattern for obtaining motion parallax information. J. Exp. Biol. 76, 237-241.

Collett, M., Despland, E., Simpson, S.J., Krakauer, D.C., 1998. Spatial scales of desert locust gregarization. Proc. Natl. Acad. Sci. U.S.A. 95, 13052-13055.

Contreras, S.C., Galindo, M.G., 2013. La plaga de la Langosta Centroamericana Schistocerca piceifrons piceifrons (Walker), en México: reconstrucción ambiental 1592-2000, in: Galindo, M.M.G., Contreras, S.C., Ibarra, Z.E. (Eds.), La plaga de la langosta Schistocerca piceifrons piceifrons Walker. Colección Sanidad Vegetal Tomo 1. Vigilancia Epidemiológica Fitosanitaria. Universidad Autónoma de San Luís Potosi, México. pp. 87-110.

Contreras, S.C., Magaña, O.C., 2013. Ficha Técnica de la Langosta Centroamericana Schistocerca piceifrons piceifrons, in: Galindo, M.M.G., Contreras, S.C., Ibarra, Z.E. (Eds.), La plaga de la langosta Schistocerca piceifrons piceifrons Walker. Colección Sanidad Vegetal Tomo 1. Vigilancia Epidemiológica Fitosanitaria. Universidad Autónoma de San Luís Potosi, México. pp. 17-36.

Cooter, R.J., 1989. Swarm flight behavior in flies and locusts, in: Goldsworthy, G., Wheeler, C. (Eds.), Insect Flight. CRC Press, Boca Raton, pp. 165-203.

Corona, M., Libbrecht, R., Wheeler, D.E., 2016. Molecular mechanisms of phenotypic plasticity in social insects. Curr. Opin. Insect Sci. 13, 55-60.

Cressman, K., 1996. Current methods of Desert Locust forecasting at FAO. OEPP/EPPO Bull. 26, $577-585$.

Cressman, K., 2013. Role of remote sensing in desert locust early warning. J. Appl. Remote Sens. 7, 075098 .

Crocker, T.D., Tschirhart, J., 1992. Ecosystems, externalities, and economies. Environ. Resource Econ. 2, 551-567.

Cruse, H., 1985. Which parameters control the leg movement of a walking insect? Part 1. Velocity control during the stance phase. J. Exp. Biol. 116, 343-355.

Cruse, H., Schmitz, J., 1983. The control-system of the femur-tibia joint in the standing leg of awalking stick insect Carausius morosus. J. Exp. Biol. 102, 175-185.

Cullen, D.A., 2012. RNAi unravels the biology of the hemimetabolous and ametabolous insects. Adv. Insect Physiol. 42, 37-72.

Cullen, D.A., Sword, G.A., Dodgson, T., Simpson, S.J., 2010. Behavioural phase change in the Australian plague locust, Chortoicetes terminifera, is triggered by tactile stimulation of the antennae. J. Insect Physiol. 56, 937-942. 
Cullen, D.A., Sword, G.A., Simpson, S.J., 2012. Optimizing multivariate behavioural syndrome models in locusts using automated video tracking. Anim. Behav. 84, 771-784.

Czirók, A., Barabasi, A.L., Vicsek, T., 1999. Collective motion of self-propelled particles: kinetic phase transition in one dimension. Phys. Rev. Lett. 82, 209.

de Vreyer, P., Guilbert, N., Mesple-Somps, S., 2015. The impact of natural disasters on education outcomes: Evidence from the 1987-89 locust plague in Mali. J. Afr. Econ. 24, 57-100.

de Wysiecki, M.L., Arturi, M, Torrusio, S., Cigliano, M.M., 2011. Influence of whether variables and plant communities on grasshopper density in the Southern Pampas, Argentina. J. Insect Sci. $11,1-14$.

de Wysiecki, M.L., Lange, C., 2005. La langosta Schistocerca cancellata Serville (Orthoptera: Acrididae: Cyrtacanthacridinae) en Argentina: biología, ecología, historia y control, in: Barrientos Lozano, L., Almaguer Sierra, P. (Eds.), Libro Manejo integrado de la langosta centroamericana (Schistocerca piceifrons piceifrons, Walker) y acridoideos plaga en América Latina. Instituto Tecnológico de Ciudad Victoria, Tamaulipas, México, pp. 151-156.

Dean, G.J.W., 1967. Observations on the structure of hopper bands and movement of hoppers of the Red Locust (Nomadacris septemfasciata Serville). J. Entomol. Soc. Sth. Afr. 30, 1-17.

Denno, R.F., Douglass, L.W., Jacobs, D., 1985. Crowding and host plant nutrition - environmental determinants of wing-form in Prokelisia marginata. Ecology 66, 1588-1596.

Despland, E., 2003. Fractal index captures the role of vegetation clumping in locust swarming. Funct. Ecol. 17, 315-322.

Despland, E., Collett, M., Simpson, S.J., 2000. Small-scale processes in desert locust swarm formation: how vegetation patterns influence gregarization. Oikos 88, 652-662.

Despland, E., Rosenberg, J., Simpson, S.J., 2004. Landscape structure and locust swarming: a satellite's eye view. Ecography 27, 381-391.

Despland, E., Simpson, S.J., 2000. The role of food distribution and nutritional quality in behavioural phase change in the Desert Locust. Anim. Behav. 59, 643-652.

Despland, E., Simpson, S.J., 2005a. Food choices of solitarious and gregarious locusts reflect cryptic and aposematic antipredator strategies. Anim. Behav. 69, 471-479.

Despland, E, Simpson, S.J., 2005b. Surviving the change to warning colouration: densitydependent polyphenism suggests a route for the evolution of aposematism. Chemoecology 15, 6975 .

Deveson, E.D., 2011. The search for a solution to Australian locust outbreaks: how developments in ecology and government responses influenced scientific research. Hist. Rec. Aust. Sci., 22, 1 31.

Deveson, E.D., 2012. Naturae Amator and the grasshopper infestations of South Australia's early years. Trans. Roy. Soc. South Aust. 136, 1-15. 
Deveson, E.D., 2013., Satellite normalized difference vegetation index data used in managing Australian plague locusts. J. Appl. Remote Sens. 7, 075096.

Deveson, E.D., Drake, V.A., Hunter, D.M., Walker, P.W., Wang, H.K., 2005. Evidence from traditional and new technologies for northward migrations of Australian plague locusts (Chortoicetes terminifera) (Walker) (Orthoptera: Acrididae) to western Queensland. Austral. Ecol. 30, 928-943.

Deveson, T., Hunter, D.M., 2002. The operation of a GIS-based decision support system for Australian locust management. Entomol. Sinica 9, 1-12.

Deveson, T., Woodman, J.D., 2014. Observations of Scelio fulgidus (Hymenoptera: Platygastridae) parasitism and development in southern NSW during the 2010 Chortoicetes terminifera (Orthoptera: Acrididae) locust plague. Austral Entomol. 53, 133-137.

Dias, B.G., Maddox, S.A., Klengel, T., Ressler, K.J., 2015. Epigenetic mechanisms underlying learning and the inheritance of learned behaviors. Trends Neurosci. 38, 96-107.

Dinku, T., Ceccato, P., Cressman, K., Connor, S.T., 2010. Evaluating detection skills of satellite rainfall estimates over desert locust recession regions. J. App. Meteorol. Climatol. 49, 1322-1332.

Dirsh, V.M., 1953. Morphometrical studies on phases of the desert locust. Anti-Locust Bull. 16, $1-34$.

Dixon, A.F.G., 2012. Aphid ecology: an optimization approach. Springer Science \& Business Media.

Drake, V.A., Gregg, P.C., Harman, I.T., Wang, H.-K., Deveson, E.D., Hunter, D.M., Rochester, W., 2001. Characterizing insect migration systems in inland Australia with novel and traditional methodologies, in: Woiwood, I.P., Reynolds, D.R., Thomas, C.D. (Eds.), Insect movement: mechanisms and consequences. Proc. Roy. Entomol. Soc. 20th Symp., CABI Publishing, London, 207-234.

Drake, V.A., Wang, H., 2013. Recognition and characterization of migratory movements of Australian plague locusts, Chortoicetes terminifera, with an insect monitoring radar. J. Appl. Remote Sens. 7, 075095.

Dreiser, U., 1994. Mapping of desert locust habitats in Africa using Landsat Thematic Mapper data. GeoJournal 32, 55-60.

Dukas, R., 2008a. Evolutionary biology of insect learning. Annu. Rev. Entomol. 53, 145-60.

Dukas, R., 2008b. Learning decreases heterospecific courtship and mating in fruitflies. Biol. Lett. 4, 645-7.

Dyson, L., Yates, C., Buhl, J., McKane, A., 2015. Onset of collective motion in locusts is captured by a minimal model. Phys. Rev. E 92, 052708.

Edney, E.B., 1937. A study of spontaneous locomotor activity in Locusta migratoria migratorioides (R. \& F.) by the actograph method. Bull. Entomol. Res. 28, 243-278. 
Ehrlich, I., Becker, G.S., 1972. Market insurance, self-insurance, and self-protection. J. Polit. Econ. 80, 623-648.

Ellis, P.E., 1951. The marching behaviour of hoppers of the African Migratory Locust (Locusta migratoria migratorioides R. \& F.) in the laboratory. Anti-Locust Bull. 7, 1-46.

Ellis, P.E., 1953. Social aggregation and gregarious behaviour in hoppers of Locusta migratoria migratorioides (R. \& F.). Behaviour 5, 225-260.

Ellis, P.E., 1959. Learning and social aggregation in locust hoppers. Anim. Behav. 7, 91-106.

Ellis, P.E., 1963a. The influence of some environmental factors on learning and aggregation in locust hoppers. Anim. Behav. 11, 142-151.

Ellis, P.E., 1963b. Changes in the social aggregation of locust hoppers with changes in rearing conditions. Anim. Behav. 11, 152-160.

Ellis, P.E., 1964a. Marching a colour in locust hoppers in relation to social factors. Behaviour 23, 177-192.

Ellis, P.E., 1964b. Changes in marching of locusts with rearing conditions. Behaviour 23, 193 202.

Ellis, P.E., Ashall, C., 1957. Field studies on diurnal behaviour, movement and aggregation in the desert locust (Schistocerca gregaria Forskål). Anti-Locust Bull. 25, 1-103.

Ely, S.O., Njagi, P.G.N., Bashir, M.O., El-Amin, S.E.T., Hassanali, A., 2011. Diel behavioral activity patterns in adult solitarious desert locust, Schistocerca gregaria (Forskål). Psyche 459315, 1-9.

Ernst, U.R., Van Hiel, M.B., Depuydt, G., Boerjan, B., De Loof, A., Schoofs, L., 2015. Epigenetics and locust life phase transitions. J. Exp. Biol. 218, 88-99.

Erra, U., Frola, B., Scarano, V., Couzin, I.D., 2009. An efficient GPU implementation for large scale individual-based simulation of collective behavior. International Workshop on High Performance Computational Systems Biology, 51-58. Doi: 10.1109/HiBi.2009.11

Escudero, C., Yates, C.A., Buhl, J., Couzin, I.D., Erban, R., Kevrekidis, I.G., Maini, P.K., 2010. Ergodic directional switching in mobile insect groups. Phys. Rev. E 82, 011926.

Evans, P.D., Bayliss, A., Reale, V., 2014. GPCR-mediated rapid, non-genomic actions of steroids: Comparisons between DmDopEcR and GPER1 (GPR30). Gen. Comp. Endocrinol. 195, 157-163.

Evans, P.D., Maqueira, B., 2005. Insect octopamine receptors: a new classification scheme based on studies of cloned Drosophila G-protein coupled receptors. Invertebrate Neurosci. 5, 111-118.

Faki, O.M.A.E., 2004. Ecological field studies on the population dynamics of the solitary Desert Locust Schistocerca gregaria (Forskål)(Acrididae: Orthoptera). Ph.D Thesis, University of Khartoum, Sudan. 
Falckenhayn, C., Boerjan, B., Raddatz, G., Frohme, M., Schoofs, L., Lyko, F., 2013. Characterization of genome methylation patterns in the desert locust, Schistocerca gregaria. J. Exp. Biol. 216, 1423-1429.

Fan, Z., Jiang, G.-F., Liu, Y.-X., He, Q.-X., Blanchard, B., 2014. Population explosion in the Yellow-spined Bamboo Locust Ceracris kiangsu and inferences for the impact of human activity. PLoS ONE 9, e89873.

Fielding, D.J., Defoliart, L.S., 2010. Embryonic developmental rates of northern grasshoppers (Orthoptera: Acrididae): Implications for climate change and habitat management. Environ. Entomol. 39, 1643-1651.

Finnoff, D., McIntosh, C., Shogren, J.F., Sims, C., Warziniack, T., 2010. Invasive species and endogenous risk. Annu. Rev. Resour. Econ. 2, 77-100.

Finnoff, D., Tschirhart, J., 2008. Linking dynamic economic and ecological general equilibrium models. Resour. Energy Econ. 30, 91-114.

Fleck, M.W., Thomson, J.L., Hough, L.B., 2012. Histamine-gated ion channels in mammals? Biochem. Pharmacol. 83, 1127-1135.

Flores, G.F., 2011. Las plagas de langosta en el área maya: ambiente e historia de una calamidad en la época prehispánica. Península 6, 27-46.

Foster, W.A., Treherne, J.E., 1981. Evidence for the dilution effect in the selfish herd from fish predation on a marine insect. Nature 293, 466-467.

Franc, A., Duranton J.-F., 2012. Red locust Nomadacris septemfasciata (Serville) upsurges in northern Madagascar between 1998 and 2004. Ann. Soc. Entomol. Fr. 48, 97-105.

Franc, A., Soti, V., Tran, A., Leclair, D., Duvallet, G., Duranton, J.-F., 2008. Deforestation, new migration pathways and outbreaks of the Red locust Nomadacris septemfasciata (Orthoptera: Acrididae) in the Sofia river basin. Conférence SDH-SAGEO, Montpellier, 23-26 June 2008, 1 17.

Fu, X.J., Hunter, D.M., Shi, W.P., 2010. Effect of Paranosema (Nosema) locustae (Microsporidia) on morphological phase transformation of Locusta migratoria manilensis (Orthoptera: Acrididae). Biocontrol Sci. Technol. 20, 683-693.

Fuchs, E., Kutsch, W., Ayali, A., 2003. Neural correlates to flight-related density dependent phase characteristics in locusts. J. Neurobiol. 57, 152-162.

Fuzeau-Braesch, S., David, J.C., 1978. Etude du taux d'octopamine chez Locusta migratoria (Insecte: Orthoptere) comparison entre insects gregaires, solitaires et traite au gaz carbonique. C. R. Acad. Sci. Série D: Sciences Naturelles 286, 697-699.

Fuzeau-Braesch, S., Nicolas, G., 1981. Effect of carbon-dioxide on subsocial insects. Comp. Biochem. Physiol. 68, 289-297. 
Gaten, E., Huston, S.J., Dowse, H.B. Matheson, T., 2012. Solitary and gregarious locusts differ in circadian rhythmicity of a visual output neuron. J. Biol. Rhythms 27, 196-205.

Geva, N., Guershon, M., Orlova, M., Ayali, A., 2010. Memoirs of a locust: Density-dependent behavioral change as a model for learning and memory. Neurobiol. Learn. Mem. 93, 175-182.

Ghaout, S., 1990. Contribution à l'étude des resources trophiques de Schistocerca gregaria (Forsk.) (Orthoptera, Acrididae) solitaire en Mauritanie occidentale et télédetection de ses biotopes par satellite. Ph.D Thesis, Université Paris XI, Orsay, France.

Giese, M., Brueck, H., Gao, Y.Z., Lin, S., Steffens, M., Kögel-Knabner, I., Glindemann, T., Susenbeth, A., Taube, F., Butterbach-Bahl, K., Zheng, X.H., Hoffmann, C., Bai, Y.F., Han, X.G., 2013. N balance and cycling of Inner Mongolia typical steppe: a comprehensive case study of grazing effects. Ecol. Monogr. 83, 195-219.

Gillett, S.D., Gonta, E., 1978. Locust as prey: factors affecting their vulnerability to predation. Anim. Behav. 26, 282-289.

Goll, M.G., Kirpekar, F., Maggert, K.A., Yoder, J.A., Hsieh, C.L., Zhang, X., Golic, K.G., Jacobsen, S.E., Bestor, T.H., 2006. Methylation of tRNA ${ }^{\text {Asp }}$ by the DNA methyltransferase homolog Dnmt2. Science 311, 395-398.

Goosey, M.W., Candy, D.J., 1980. The D-octopamine content of the haemolymph of the locust Schistocerca americana gregaria and its elevation during flight. Insect Biochem. 10, 393-397.

Gordon, S.D., Jackson, J.C., Rogers, S.M., Windmill, J.F.C., 2014. Listening to the environment: hearing differences from an epigenetic effect in solitarious and gregarious locusts. Proc. Roy. Soc. B Biol. Sci. 281, 20141693.

Gornyy, V.I., Kritsuk, S.G., Latypov, I.S., Teplyakova, T.E., Tronin A.A., 2011. Measuring approach of satellite locust monitoring. J. Earth Sci. Eng. 1, 126-131.

Gotham, S., Song, H., 2013. Non-swarming grasshoppers exhibit density-dependent phenotypic plasticity reminiscent of swarming locusts. J. Insect Physiol. 59, 1151-1159.

Gray, L.J., Sword, G.A., Anstey, M.L., Clissold, F.J., Simpson, S.J., 2009. Behavioural phase polyphenism in the Australian plague locust (Chortoicetes terminifera). Biol. Lett. 5, 306-309.

Greenwood, M., Chapman, R.F., 1984. Differences in numbers of sensilla on the antennae of solitarious and gregarious Locusta migratoria L. (Orthoptera: Acrididae). Int. J. Insect Morph. Embryol. 13, 295-301.

Grohmann, L., Blenau, W., Erber, J., Ebert, P.R., Strünker, T., Baumann, A., 2003. Molecular and functional characterization of an octopamine receptor from honeybee (Apis mellifera) brain. J. Neurochem. 86, 725-735.

Guo, W., Wang, X., Ma, Z., Xue, L., Han, J., Yu, D., Kang, L., 2011. CSP and takeout genes modulate the switch between attraction and repulsion during behavioral phase change in the migratory locust. PLoS Genet. 7, e1001291. 
Guo, X., Ma, Z., Kang, L., 2013. Serotonin enhances solitariness in phase transition of the migratory locust. Front. Behav. Neurosci. 7, 129.

Guo, X., Ma, Z., Kang, L., 2015. Two dopamine receptors play different roles in phase change of the migratory locust. Front. Behav. Neurosci. 9, 80.

Guttal, V., Romanczuk, P., Simpson, S.J., Sword, G.A., Couzin, I.D., 2012. Cannibalism can drive the evolution of behavioural phase polyphenism in locusts. Ecol. Lett. 15, 1158-1166.

Hamilton, W.D., 1971. Geometry for the selfish herd. J. Theor. Biol. 31, 295-311.

Han, K.A., Millar, N.S., Grotewiel, M.S., Davis, R.L., 1996. DAMB, a novel dopamine receptor expressed specifically in Drosophila mushroom bodies. Neuron 16, 1127-1135.

Hansen, M.J., Buhl, J., Bazazi, S., Simpson, S.J., Sword, G.A., 2011. Cannibalism in the lifeboatcollective movement in Australian plague locusts. Behav. Ecol. Sociobiol. 65, 1715-1720.

Hardie, R.C., 1989. A histamine-activated chloride channel involved in neurotransmission at a photoreceptor synapse. Nature 339, 704-06.

Harrell Jr., F.E., 2001. Regression Modeling Strategies: With Applications to Linear Models, Logistic Regression, and Survival Analysis. Springer, New York.

Harvey, A.W., 1983. Schistocerca piceifrons (Walker) (Orthoptera: Acrididae), the swarming locust of tropical America: a review. Bull. Entomol. Res. 73, 171-184.

Hassenstein, B., Hustert, R., 1999. Hiding responses of locusts to approaching objects. J. Exp. Biol. 202, 1701-1710.

Hauser, F., Cazzamali, G., Williamson, M., Park, Y., Li, B., Tanaka, Y., Predel, R., Neupert, S., Schachtner, J., Verleyen, P., Grimmelikhuijzen, C.J., 2008. A genome-wide inventory of neurohormone GPCRs in the red flour beetle Tribolium castaneum. Front. Neuroendocrinol. 29, $142-165$.

Hauser, F., Cazzamali, G., Williamson, M., Blenau, W., Grimmelikhuijzen, C.J., 2006. A review of neurohormone GPCRs present in the fruitfly Drosophila melanogaster and the honey bee Apis mellifera. Prog. Neurobiol. 80, 1-19.

Hearn, M.G., Ren, Y., McBride, E.W., Reveillaud, I., Beinborn, M., Kopin, A.S., 2002. A Drosophila dopamine 2-like receptor: Molecular characterization and identification of multiple alternatively spliced variants. Proc. Natl. Acad. Sci. U.S.A. 99, 14554-14559.

Heifetz, Y., 1997. The control of phase transition in the desert locust Schistocerca gregaria (Forskål). Ph.D Thesis, Hebrew University of Jerusalem, Israel.

Heifetz, Y., Applebaum, S.W., 1995. Density-dependent physiological phase in a non-migratory grasshopper Aiolopus thalassinus. Entomol. Exp. Appl. 77, 251-262.

Hernández-Zul, M.I., Quijano-Carranza, J.A., Yañez-López, R., Ocampo-Velazquez, R.V., Torrés-Pacheco, I., Guevara-Gonzalez, R.G., Castro Ramírez, A.E., 2013. Dynamic simulation 
model of Central American Locust Schistocerca piceifrons (Othoptera: Acrididae). Fla. Entomol. 96, 1274-1283.

Hielkema, J.U., 1981. Desert locust habitat monitoring with satellite remote sensing: A new technology for an old problem. ITC Journal 4, 387-417.

Hielkema, J.U., Roffey, J., Tucker, C.J., 1986. Assessment of ecological conditions associated with the 1980/81 desert locust plague upsurge in West Africa using environmental satellite data. Int. J. Remote Sens. 7, 1609-1622.

Hill, C.A., Doyle, T., Nuss, A.B., Ejendal, K.F.K., Meyer, J.M., Watts, V.J., 2016. Comparative pharmacological characterization of D1-like dopamine receptors from Anopheles gambiae, Aedes aegypti and Culex quinquefasciatus suggests pleiotropic signaling in mosquito vector lineages. Parasit. Vectors 9, 192.

Hosmer, D. W., Hosmer, T., Le Cessie, S., Lemeshow, S., 1997. A comparison of goodness-of-fit tests for the logistic regression model. Stat. Med. 16, 965-980.

Hoste, B., Simpson, S.J., Tanaka, S., De Loof, A., Breuer, M., 2002. A comparison of phaserelated shifts in behavior and morphometrics of an albino strain, deficient in [His7]-corazonin, and a normally colored Locusta migratoria strain. J. Insect Physiol. 48, 791-801.

Houghton, J.T., Ding, Y., Griggs, D.J., Noguer, N., van der Linden, P.J., Xiaosu, D., Maskell, K., Johnson, C.A. (Eds.), 2001. Climate Change 2001: The Scientific Basis. Cambridge University Press, Cambridge, $881 \mathrm{pp}$.

Huang, J., Hamasaki, T., Ozoe, Y., 2010. Pharmacological characterization of a Bombyx mori alpha-adrenergic-like octopamine receptor stably expressed in a mammalian cell line. Arch. Insect Biochem. Physiol. 73, 74-86.

Huang, J., Manning, B.D., 2009. A complex interplay between Akt, TSC2 and the two mTOR complexes. Biochem. Soc. Trans. 37, 217-222.

Humphries, M.A., Mustard, J.A., Hunter, S.J., Mercer, A., Ward, V., Ebert, P.R., 2003. Invertebrate D2 type dopamine receptor exhibits age-based plasticity of expression in the mushroom bodies of the honeybee brain. J. Neurobiol. 55, 315-330.

Hunter, D.M., 2004. Advances in the control of locusts (Orthoptera: Acrididae) in eastern Australia: from crop protection to preventative control. Austral. J. Entomol., 43, 293-303.

Hunter, D., Cosenzo, E., 1990. The origin of plagues and recent outbreaks of the South American locust, Schistocerca cancellata (Orthoptera: Acrididae) in Argentina. Bull. Entomol. Res. 80, 295 300 .

Hunter, D.M., McCulloch, L., Spurgin, P.A., 2008. Aerial detection of nymphal bands of the Australian plague locust (Chortoicetes terminifera (Walker)(Orthoptera: Acrididae). Crop Prot., $27,118-123$. 
Hunter, D., McCulloch, L., Wright, D., 1981. Lipid accumulation and migratory flight in the Australian plague locust, Chortoicetes terminifera (Walker)(Orthoptera: Acrididae). Bull. Entomol. Res. 71, 543-546.

IPPC, 2007. Climate change 2007. Synthesis Report. Contribution of Working Groups I, II and III to the Fourth Assessment Report of the Intergovernmental Panel on Climate Change. Pachauri R.K., Reisinger A. (Eds.). IPCC, Geneva, Switzerland, 104 pp.

Isles, A.R., 2015. Neural and behavioral epigenetics; what it is, and what is hype. Genes Brains Behav. 14, 64-72.

Jech, L., 2015. Grasshoppers in Arizona in 2015. Proceedings of Annual Meeting of the National Grasshopper Management Board, Denver, CO.

Jeltsch, A., Ehrenhofer-Murray, A., Jurkowski, T.P., Lyko, F., Reuter, G., Ankri, S., Nellen, W., Schaefer, M., Helm, M., 2016. Mechanism and biological role of Dnmt2 in nucleic acid methylation. RNA Biol. doi: 10.1080/15476286.2016.1191737.

Ji, R., Xie, B.Y., Li, D.M., Li, Z., Zhang, X., 2004. Use of MODIS data to monitor the oriental migratory locust plague. Agr. Ecosyst. Environ. 104, 615-620.

Jiang, F., Yang, M., Guo, W., Wang, X., Kang, L., 2012. Large-scale transcriptome analysis of retroelements in the Migratory Locust, Locusta migratoria. PLoS ONE 7, e40532.

Johnson, C., 1969. Migration and dispersal of insects by flight. Methuen, London.

Kane, S., Shogren, J.F., 2000. Linking adaptation and mitigation in climate change policy. Climatic Change 45, 75-102.

Kang, L., Chen, X., Zhou, Y., Liu, B., Zheng, W., Li, R., Wang, J., Yu, J., 2004. The analysis of large-scale gene expression correlated to the phase changes of the migratory locust. Proc. Natl. Acad. Sci. U.S.A. 101, 17611-17615.

Kay, R.D., Edwards, W.M., Duffy, P.A., 2012. Farm Management, seventh ed. McGraw-Hill Education, New York.

Key, K., 1950. A critique on the phase theory of locusts. Q. Rev. Biol. 25, 363-407.

Kibasa, R., 2006. Use of SPOT 5 Image to identify presence of locusts. International Institute for Geoinformation Science and Earth Observation, Enschede, Netherlands.

Kita, T., Irie, T., Nomura, K., Ozoe, F., Ozoe, Y., 2017. Pharmacological characterization of histamine-gated chloride channels from the housefly Musca domestica. Neurotoxicol. 60, 245253.

Koh, L.P., Wich, S.A., 2012. Dawn of drone ecology: low-cost autonomous vehicles for conservation. Trop. Conserv. Sci. 5, 121-132.

Kuitert, L.C., Connin, R.V., 1952. Biology of the American grasshopper in the southeastern United States. Fla. Entomol. 35, 22-33. 
Latchininsky, A.V., 2013. Locusts and remote sensing: a review. J. Appl. Remote Sens. 7, 075099.

Latchininsky, A.V., 2017a. Climate change and locusts: what to expect? Proc. Russian State Hydrometeorolog. Univ. 46, 134-143.

Latchininsky, A.V., 2017b. Locusts, in: Reference Module in Life Sciences, Elsevier.

Latchininsky, A.V., Kokanova, E.O., Gapparov, F.A., Childebaev, M.K., Temreshev, I.I., 2015. Locusts and climate change. Bull. Al-Farabi Kazakhstan State Univ., Ecology Series 2/2 (44), 641648 (in Russian with English summary).

Latchininsky, A.V., Launois-Luong, M.H., 1992. Le Criquet marocain, Dociostaurus maroccanus (Thunberg, 1815), dans la partie orientale de son aire de distribution. Etude monographique relative à l'ex-URSS et aux pays proches, International Center of Agronomic Research for Development CIRAD-GERDAT-PRIFAS, Montpellier, France / All-Russian Research Institute for Plant Protection VIZR, St. Petersburg, Russia.

Latchininsky, A.V., Piou, C., Franc, A., Soti, V., 2016. Applications of Remote Sensing to Locust Management, in: Baghdadi N., Zribi M. (Eds.), Land Surface Remote Sensing, Environment and Risks. ISTE Press/Elsevier, London/Oxford, pp. 263-293.

Latchininsky, A.V., Sivanpillai, R., 2010. Locust habitat monitoring and risk assessment using remote sensing and GIS technologies, in: Ciancio, A., Mukerji, K.G. (Eds.), Integrated Management of Arthropod Pests and Insect Borne Diseases, Springer, Dordrecht-HeidelbergLondon-New York, pp. 163-188.

Latchininsky, A.V., Sivanpillai, R., Driese, K.L., Wilps, H., 2007. Can early season Landsat image improve locust habitat monitoring in the Amudarya River Delta, Uzbekistan? J. Orthoptera Res. $16,167-173$.

Lechner, M., Marz, M., Ihling, C., Sinz, A., Stadler, P.F., Krauss, V., 2013. The correlation of genome size and DNA methylation rate in metazoans. Theor. Biosci. 132, 47-60.

Lecoq, M., 2005. Desert locust management: from ecology to anthropology. J. Orthoptera Res. 14, 179-186.

Lecoq, M., Balanca, G., 1998. Field trials of fipronil for control of Rhammatocerus schistocercoides (Rehn, 1906) hopper bands in Brazil. Crop Prot. 17, 105-110.

Lecoq, M., Foucart, A., Balanca, G., 1999. Behaviour of Rhammatocerus schistocercoides (Rehn, 1096) hopper bands in Mato Grosso, Brazil (Orthoptera: Acrididae : Gomphocerinae). Ann. Soc. Entomol. Fr. 35, 217-228.

Leitch, B., Judge, S., Pitman, R.M., 2003. Octopaminergic modulation of synaptic transmission between an identified sensory afferent and flight motoneuron in the locust. J. Comp. Neurol. 462, $55-70$.

Lenoir, J., Gégout, J.C., Marquet, P.A., de Ruffray, P., Brisse, H., 2008. A significant upward shift in plant species optimum elevation during the $20^{\text {th }}$ Century. Science 320, 1768-1771. 
Libersat, F., Pflueger, H.-J., 2004. Monoamines and the orchestration of behavior. BioScience 54, $17-25$.

Lihoreau, M., Raine, N.E., Reynolds, A.M., Stelzer, R.J., Lim, K.S., Smith, A.D., Osborne, J.L., Chittka, L., 2012. Radar tracking and motion-sensitive cameras on flowers reveal the development of pollinator multi-destination routes over large spatial scales. PLoS Biol. 10, e1001392.

Liu, X., 1975. Old Book of Tang. Zhonghua Book Company, Beijing, China.

Lockwood, J.A., Showler, A.T., Latchininsky, A.V., 2001. Can we make locust and grasshopper management sustainable? J. Orthoptera Res. 10, 315-329.

Lorenz, M.W., 2009. Migration and trans-Atlantic flight of locusts. Quatern. Int. 196, 4-12.

Louveaux, A., Ghaout, S., Gillon, Y., 1990. Fonctionnement de l'aire de reproduction hivernale du criquet pèlerin en Mauritanie, ANPP - Deuxième Conférence Internationale sur les Ravageurs de l'Agriculture. ANPP, Versailles, France.

Lovejoy, N.R., Mullen, S.P., Sword, G.A., Chapman, R.F., Harrison, R.G., 2006. Ancient transAtlantic flight explains locust biogeography: molecular phylogenetics of Schistocerca. Proc. Roy. Soc. B Biol. Sci. 273, 767-774.

Löw, F., Waldner, F., Latchininsky, A.V., Biradar, C., Bolkart, M., Colditz, R., 2016. Timely monitoring of Asian Migratory locust habitats in the Amudarya delta, Uzbekistan using time series of satellite remote sensing vegetation index. J. Environ. Manage. 183, 562-575.

Löw, F., Navratil, P., Kotte, K., Schöler, H.F., Bubenzer, O., 2013. Remote sensing based analysis of landscape change in the desiccated seabed of the Aral Sea - a potential tool for assessing the hazard degree of dust and salt storms. Environ. Monit. Assess. 185, 8303-8319.

Lyko, F., Foret, S., Kucharski, R., Wolf, S., Falckenhayn, C., Maleszka, R., 2010. The honey bee epigenomes: differential methylation of brain DNA in queens and workers. PLoS Biol. 8, e1000506.

Ma, S., 1958. The population dynamics of the Oriental migratory locust (Locusta migratoria manilensis Meyen) in China. Acta Entomol. Sin. 8, 1-40 (in Chinese).

Ma, C., Yang, P., Jiang, F., Chapuis, M.P., Shali, Y., Sword, G.A., Kang, L., 2012. Mitochondrial genomes reveal the global phylogeography and dispersal routes of the migratory locust. Mol. Ecol. $21,4344-4358$.

Ma, J., Han, X., Hasibagan, Wang, C., Zhang, Y., Tang, J., Xie, Z., Deveson, T., 2005. Monitoring East Asian migratory locust plagues using remote sensing data and field investigations. Int. J. Remote Sens. 26, 629-634.

Ma, Z., Guo, W., Guo, X., Wang, X., Kang, L., 2011. Modulation of behavioral phase changes of the migratory locust by the catecholamine metabolic pathway. Proc. Natl. Acad. Sci. U.S.A. 108, 3882-3887. 
Ma, Z., Guo, X., Lei, H., Li, T., Hao, S., Kang, L., 2015. Octopamine and tyramine respectively regulate attractive and repulsive behavior in locust phase changes. Sci. Rep. 5, 8036.

Ma, Z., Yu, J., Kang, L., 2006. LocustDB: a relational database for the transcriptome and biology of the migratory locust (Locusta migratoria). BMC Genomics 7, 11.

Maeno, K.O., Ely, S.O., Nakamura, S., Abdellaoui, K., Cissé, S., El Hacen Jaavar, M., Mohamed, S.A.O., Atheimine, M., Babah, M.A.O., 2016. Daily microhabitat shifting of solitarious-phase desert locust adults: implications for meaningful population monitoring. Springerplus, 5, 107.

Maeno, K.O., Piou, C., Ely, S.O., Mohamed, S.O., Jaavar, M.E.H., Babah, M.A.O., Nakamura, S., 2012. Field observations of the sheltering behavior of the solitarious phase of the desert locust, Schistocerca gregaria, with particular reference to antipredator strategies. JARQ 46, 339-345.

Maeno, K.O., Piou, C., Babah, M.A.O., Nakamura, S., 2013. Eggs and hatchlings variations in desert locusts: phase related characteristics and starvation tolerance. Front. Physiol. 4, 345.

Maeno, K., Tanaka, S., 2011. Phase-specific responses to different qualities of food in the desert locust, Schistocerca gregaria: Developmental, morphological and reproductive characteristics. J. Insect Physiol. 57, 514-520.

Magor, J.I., Lecoq, M., Hunter, D.M., 2008. Preventive control and Desert Locust plagues. Crop Prot. 27, 1527-1533.

Mallon, E.B., Amarasinghe, H.E., Ott, S.R., 2016. Acute and chronic gregarisation are associated with distinct DNA methylation fingerprints in desert locusts. Sci. Rep. 6, 35608.

Maqueira, B., Chatwin, H.M., Evans, P.D., 2005. Identification and characterization of a novel family of Drosophila beta-adrenergic-like octopamine G-protein coupled receptors. J. Neurochem. 94, 547-560.

Marvin, D.C., Koh, L.P., Lynam, A.J., Wich, S., Davies, A.B., Krishnamurthy, R., Stokes, E., Starkey, R., Asner, G.P., 2016. Integrating technologies for scalable ecology and conservation. Global Ecol. Conserv. 7, 262-275.

Matheson, T., Rogers, S.M., Krapp, H.G., 2004. Plasticity in the visual system is correlated with a change in lifestyle of solitarious and gregarious locusts. J. Neurophys. 91, 1-12.

McCulloch, L., Hunter, D.M., 1983. Identification and monitoring of Australian plague locust habitats from Landsat. Remote Sens. Environ. 13, 95-102.

Mery, F., Kawecki, T.J., 2004. An operating cost of learning in Drosophila melanogaster. Anim. Behav. 68, 589-598.

Mery, F., Kawecki, T.J., 2005. A cost of long-term memory in Drosophila. Science 308, 1148.

Michel, R., 1970. Étude expérimentale des variations de la tendance au vol chez le criquet pèlerin Schistocerca gregaria (Forsk), élevé isolément pendant plusieurs générations. Insectes Sociaux Paris 17, 21-38. 
Missale, C., Nash, S.R., Robinson, S.W., Jaber, M., Caron, M.G., 1998. Dopamine receptors: from structure to function. Physiol. Rev. 78, 189-225.

Müller, C.B., Williams, I.S., Hardie, J., 2001. The role of nutrition, crowding and interspecific interactions in the development of winged aphids. Ecol. Entomol. 26, 330-340.

Musolin, D.L., Saulich, A.H., 2012. Reactions of insects to recent climate change: from physiology and behavior to distribution area shift. Entomol. Rev. 91, 3-35 (in Russian with English summary).

Mustard, J.A., Blenau, W., Hamilton, I.S., Ward, V.K., Ebert, P.R., Mercer, A.R., 2003. Analysis of two D1-like dopamine receptors from the honey bee Apis mellifera reveals agonist-independent activity. Mol. Brain Res. 113, 67-77.

Mustard, J.A., Beggs, K.T., Mercer, A.R., 2005. Molecular biology of the invertebrate dopamine receptors. Arch. Insect Biochem. Physiol. 59, 103-117.

Navratil, P., Wilps, H., 2013. Object-based locust habitat mapping using high resolution multispectral satellite data in the southern Aral Sea basin. J. Appl. Remote Sens. 7, 075097.

Nespolo, R.F., Roff, D.A., Fairbairn, D.J., 2008. Energetic trade-off between maintenance costs and flight capacity in the sand cricket (Gryllus firmus). Funct. Ecol. 22, 624-631.

Nichols, D.E., Nichols, C.D., 2008. Serotonin receptors. Chem. Rev. 108, 1614-1641.

Ngumbi, E., Jordan, M., Fadamiro, H., 2012. Comparison of associative learning of host-related plant volatiles in two parasitoids with different degrees of host specificity, Cotesia marginiventris and Microplitis croceipes. Chemoecology 22, 207-215.

Nishide, Y., Tanaka, S., 2016. Desert locust, Schistocerca gregaria, eggs hatch in synchrony in a mass but not when separated. Behav. Ecol. Sociobiol. 70, 1507-1515.

Nishide, Y., Tanaka, S., Saeki, S., 2015a. Adaptive difference in daily timing of hatch in two locust species, Schistocerca gregaria and Locusta migratoria: The effects of thermocycles and phase polyphenism. J. Insect Physiol. 72, 79-87.

Nishide, Y., Tanaka, S., Saeki, S., 2015b. Egg hatching of two locusts, Schistocerca gregaria and Locusta migratoria, in response to light and temperature cycles. J. Insect Physiol. 76, 24-29.

Noldus, L.P.J.J., Spink, A.J., Tegelenbosch, R.A.J, 2001. Etho Vision: A versatile video tracking system for automation of behavioral experiments. Behav. Res. Methods. Instrum. Comput. 33, 398-414.

Nolte, D., 1967. Phase transformation and chiasma formation in locusts. Chromosoma 21, 123139.

Nufio, C.R., McGuire, C.R., Bowers, M.D., Guralnick, R.P., 2010. Grasshopper community response to climatic change: variation along an elevational gradient. PLoS ONE, 5, e12977. 
Ochieng', S.A., Hallberg, E., Hansson, B.S., 1998. Fine structure and distribution of antennal sensilla of the desert locust, Schistocerca gregaria (Orthoptera: Acrididae). Cell Tissue Res. 291, $525-536$.

Oh, Y., Jang, D., Sonn, J.Y., Choe, J., 2013. Histamine-HisC11 receptor axis regulates wakepromoting signals in Drosophila melanogaster. PLoS ONE 8, e68269.

Ohta, H., Utsumi, T., Ozoe, Y., 2003. B96Bom encodes a Bombyx mori tyramine receptor negatively coupled to adenylate cyclase. Insect Mol. Biol. 12, 217-223.

Ohta, H., Tsuchihara, K., Mitsumasu, K., Yaginuma, T., Ozoe, Y., Asaoka, K., 2009. Comparative pharmacology of two D1-like dopamine receptors cloned from the silkworm Bombyx mori. Insect Biochem. Mol. Biol. 39, 342-347.

Ohtani, A., Arai, Y., Ozoe, F., Ohta, H., Narusuye, K., Huang, J., Enomoto, K., Kataoka, H., Hirota, A., Ozoe, Y., 2006. Molecular cloning and heterologous expression of an alpha-adrenergiclike octopamine receptor from the silkworm Bombyx mori. Insect Mol. Biol. 15, 763-772.

Olfert, O., Weiss, R.M., Kriticos, D., 2011. Application of general circulation models to assess the potential impact of climate change on potential distribution and relative abundance of Melanoplus sanguinipes (Fabricius) (Orthoptera: Acrididae) in North America. Psyche, 980372.

Oppenheim, S.J., Baker, R.H., Simon, S., DeSalle, R., 2015. We can't all be supermodels: the value of comparative transcriptomics to the study of non-model insects: Comparative transcriptomics of non-model insects. Insect Mol. Biol. 24, 139-154.

Ostrom, E., 2009. A general framework for analyzing sustainability of social-ecological systems. Science 325, 419-422.

Ott, S.R., Verlinden, H., Rogers, S.M., Brighton, C.H., Quah, P.S., Vleugels, R.K., Verdonck, R., Vanden Broeck, J., 2012. Critical role for protein kinase A in the acquisition of gregarious behavior in the desert locust. Proc. Natl. Acad. Sci. U.S.A. 109, 381-387.

Padgham, D.E., 1981. Hatching rhythms in the desert locust, Schistocerca gregaria. Physiol. Entomol. 6, 191-198.

Pantazis, A., Segaran, A., Liu, C.H., Nikolaev, A., Rister, J., Thum, A.S., Roeder, T., Semenov, E., Juusola, M., Hardie, R.C., 2008. Distinct roles for two histamine receptors (hclA and hclB) at the Drosophila photoreceptor synapse. J. Neurosci. 28, 7250-7259.

Pedgley, D.E., 1974. ERTS surveys a $500 \mathrm{~km}^{2}$ locust breeding site in Saudi Arabia, in: Frieden S.C., Mercanti E.P., Becker M.A. (Eds.), Third Earth Resources Technology Satellite Symposium (December 1973), NASA, Maryland, Vol. 1, 233-246.

Pekel, J.F., Ceccato, P., Vancutsem, C., Cressman, K., Vanbogaert, E., Defourny, P., 2011. Development and application of multi-temporal colorimetric transformation to monitor vegetation in the Desert Locust habitat. IEEE J. STARS 4, 318-326.

Pélissié, B., Piou, C., Jourdan-Pineau, H., Pagès, C., Blondin, L., Chapuis, M.-P., 2016. Extra molting and selection on nymphal growth in the Desert Locust. PLoS ONE 11, e0155736. 
Péloquin, C., 2014. Unruly nature and technological authority: Governing locust swarms in the Sahel. Ph.D Thesis, University of Arizona, Tempe, AZ, U.S.A.

Pener, M.P., 1983. Endocrine aspects of phase polymorphism in locusts, in: Downer, R.G.H., Laufer, H. (Eds.), Invertebrate Endocrinology, Vol. 1, Endocrinology of Insects. Alan R. Liss Inc., New York, pp. 379-394.

Pener, M.P., 1991. Locust phase polymorphism and its endocrine relations. Adv. Insect Physiol. 23, $1-79$.

Pener, M.P., Ayali, A., Golenser, E., 1997. Adipokinetic hormone and flight fuel related characteristics of density-dependent locust phase polymorphism: A review. Comp. Biochem. Physiol. B 117, 513-524.

Pener, M.P., Simpson, S.J., 2009. Locust phase polyphenism: an update. Adv. Insect Physiol. 36, 1-286.

Pener, M.P., Yerushalmi, Y., 1998. The physiology of locust phase polymorphism: an update. J. Insect Physiol. 44, 365-377.

Penick, C.A., Brent, C.S., Dolezal, K., Liebig, J., 2014. Neurohormonal changes associated with ritualized combat and the formation of a reproductive hierarchy in the ant Harpegnathos saltator. J. Exp. Biol. 217, 1496-1503.

Peroutka, S.J., Howel, T.A., 1994. The molecular evolution of G protein-coupled receptors: focus on 5-hydroxytryptamine receptors. Neuropharmacol. 33, 319-24.

Pfadt, R.E., 1988. Field Guide to the Common Western Grasshoppers. University of Wyoming Agricultural Experiment Station Bull. 912, 145 pp.

Pfluger, J-H., Stevenson, P.A, 2005. Evolutionary aspects of octopaminergic systems with emphasis on arthropods. Arthropod Struct. Dev. 34, 379-396.

Piou, C., Lebourgeois, V., Benahi, A.S., Bonnal, V., Jaavar, M.E.H., Lecoq, M., Vassal, J.M., 2013. Coupling historical prospection data and a remotely-sensed vegetation index for the preventative control of Desert locusts. Basic Appl. Ecol. 14, 593-604.

Poels, J., Suner, M.M., Needham, M., Torfs, H., De Rijck, J., De Loof, A., Dunbar, S.J., Vanden Broeck, J., 2001. Functional expression of a locust tyramine receptor in murine erythroleukaemia cells. Insect Mol. Biol. 10, 541-48.

Poot-Pech, M.A., 2016. La langosta voladora Schistocerca piceifrons (Orthoptera: Acrididae): hacia un manejo sustentable. In: El patrimonio, su importancia y conservación. Conociendo el patrimonio, Cuerpo académico patrimonio y desarrollo sustentable, Editorial TECCIS, San Francisco de Campeche, 58-66.

Poot-Pech, M.A., 2017. Meeting on the locust situation in South America and the OIRSA region. Metaleptea 37, 2. 
Poot-Pech, M.A., Ruíz, S.E., Ballina, G.H., 2017. Indicator plants in solitary phase and migration behavior of Schistocerca piceifrons in Yucatán, México. Metaleptea 37, 21.

Poot-Pech, M.A., Ruiz-Sánchez, E., Ballina-Gómez, H.S., Gamboa-Angulo, M.M., ReyesRamírez, A., 2016. Olfactory response and host plant feeding of the Central American Locust Schistocerca piceifrons piceifrons Walker to common plants in a gregarious zone. Neotrop. Entomol. 45, 382-388.

Popov, G.B., Duranton, J.-F., Gigault, J., 1991. Etude écologique des biotopes du criquet pèlerin Schistocerca gregaria (Forskal, 1775) en Afrique Nord-Occidentale: mise en évidence et description des unités territoriales écologiquement homogènes CIRAD-PRIFAS, Montpellier.

Popova, E.N., 2014. The increase in locust threat following climate warming in Russia: calculating estimations. Agro XXI, 7-9.

Popova, E.N., Semenov, S.M., Popov, I.O., 2016. Assessment of possible expansion of the climatic range of Italian locust (Calliptamus italicus L.) in Russia in the $21^{\text {st }}$ century at simulated climate changes. Russian Meteorol. Hydrol. 41, 213-217.

Propastin, P., 2012. Multisensor monitoring system for assessment of locust hazard risk in the Lake Balkhash drainage basin. Environ. Manage. 50, 1234-1246.

Propastin, P., 2013. Satellite-based monitoring system for assessment of vegetation vulnerability to locust hazard in the River Ili delta (Lake Balkhash, Kazakhstan). J. Appl. Remote Sens. 7, 075094.

Qi, Y.X., Xia, R.Y., Wu, Y.S., Stanley, D., Huang, J., Ye, G.Y., 2014. Larvae of the small white butterfly, Pieris rapae, express a novel serotonin receptor. J. Neurochem. 131, 767-77.

Raszick, T.J., Song, H., 2016. The ecotype paradigm: testing the concept in an ecologically divergent grasshopper. Insect Syst. Evol. 47, 363-387.

Reale, V., Hannan, F., Hall, L.M., Evans, P.D., 1997. Agonist-specific coupling of a cloned Drosophila melanogaster D1-like dopamine receptor to multiple second messenger pathways by synthetic agonists. J. Neurosci. 17, 6545-6553.

Reim, T., Balfanz, S., Baumann, A., Blenau, W., Thamm, M., Scheiner, R. AmTAR2: Functional characterization of a honeybee tyramine receptor stimulating adenylyl cyclase activity. Insect Biochem. Mol. Biol. 80, 91-100.

Renier, C., Waldner, F., Jacques, D., Babah Ebbe, M., Cressman, K., Defourny, P., 2015. A dynamic vegetation senescence indicator for near-real-time desert locust habitat monitoring with MODIS. Remote Sens. 7, 7545-7570.

Reynolds, D.R., 1988. Twenty years of radar entomology. Antenna 12, 44-49.

Riley, J.R., 1989. Remote sensing in entomology. Annu. Rev. Entomol. 34, 247-271.

Riley, J.R., Greggers, U., Smith, A.D., Reynolds, D.R., Menzel, R., 2005. The flight paths of honeybees recruited by the waggle dance. Nature 435, 205-207. 
Riley, J.R., Reynolds, D.R., 1997. Vertical-looking radar as a means to improve forecasting and control of desert locusts, in: Krall, S., Peveling, R. Ba Diallo, D. (Eds.), New strategies in locust control. Birkhauser Verlag, Basel, pp. 47-54.

Rillich, J., Stevenson, P.A., 2014. A fighter's comeback: dopamine is necessary for recovery of aggression after social defeat in crickets. Horm. Behav. 66, 696-704.

Robb, S., Cheek, T.R., Hannan, F.L., Hall, L.M., Midgley, J.M., Evans, P.D., 1994. Agonistspecific coupling of a cloned Drosophila octopamine/tyramine receptor to multiple second messenger systems. EMBO J. 13, 1325-1330.

Robinet, C., Roques, A., 2010. Direct impacts of recent climate warming on insect populations. Integr. Zool. 5, 132-142.

Robinson, K.L., Tohidi-Esfahani, D., Lo, N., Simpson, S.J., Sword G.A., 2011. Evidence for widespread genomic methylation in the migratory locust, Locusta migratoria (Orthoptera: Acrididae). PLoS ONE 6, e28167.

Robinson, K.L., Tohidi-Esfahani, D., Ponton, F., Simpson, S.J., Sword, G.A., Lo, N., 2016. Alternative migratory locust phenotypes are associated with differences in the expression of genes encoding the methylation machinery. Insect Mol. Biol. 25, 105-115.

Roessingh, P., Bouaïchi, A., Simpson, S.J., 1998. Effects of sensory stimuli on the behavioural phase state of the desert locust, Schistocerca gregaria. J. Insect Physiol. 44, 883-893.

Roessingh, P., Simpson, S.J., 1994. The time-course of behavioural phase change in nymphs of the desert locust, Schistocerca gregaria. Physiol. Entomol. 19, 191-197.

Roessingh, P., Simpson, S.J., James, S., 1993. Analysis of phase-related changes in behavior of desert locust nymphs. Proc. Roy. Soc. B Biol. Sci. 252, 43-49.

Roffey, J., 1963. Observation on night flight in the desert locust Schistocerca gregaria (Forskål). Anti-Locust Bull. 39, 1-32.

Roffey, J., 1969. Report on radar studies on the desert locust, Schistocerca gregaria (Forskål) in the Niger Republic, (September-October 1968). Occasional Report no. 17, Anti-Locust Research Centre, London.

Roffey, J., Popov, G., 1968. Environmental and behavioural processes in a desert locust outbreak. Nature 219, 446-450.

Rogers, S.M., Cullen, D.A., Anstey, M.L., Burrows, M., Despland, E., Dodgson, T., Matheson, T., Ott, S.R., Stettin, K., Sword, G.A., Simpson S.J., 2014. Rapid behavioural gregarization in the desert locust, Schistocerca gregaria entails synchronous changes in both activity and attraction to conspecifics. J. Insect Physiol. 65, 9-26.

Rogers, S.M., Harston, G.W.J., Kilburn-Toppin, F., Matheson, T., Burrows, M., Gabbiani, F., Krapp, H.G., 2010. Spatiotemporal receptive field properties of a looming-sensitive neuron in solitarious and gregarious phases of the desert locust. J. Neurophys. 103, 779-792. 
Rogers, S.M., Krapp, H.G., Burrows, M., Matheson, T., 2007. Compensatory plasticity at an identified synapse tunes a visuomotor pathway. J. Neurosci. 27, 4621-4633.

Rogers, S.M., Matheson, T., Despland, E., Dodgson, T., Burrows, M., Simpson, S.J., 2003. Mechanosensory-induced behavioural gregarization in the desert locust Schistocerca gregaria. J. Exp. Biol. 206, 3991-4002.

Rogers, S.M., Matheson, T., Sasaki, K., Kendrick, K., Simpson, S.J., Burrows, M., 2004. Substantial changes in central nervous system neurotransmitters and neuro-modulators accompany phase change in the locust. J. Exp. Biol. 207, 3603-3617.

Rogers, S.M., Ott, S.R., 2015. Differential activation of serotonergic neurons during short- and long-term gregarization of desert locusts. Proc. Roy. Soc. B Biol. Sci. 282, 20142062.

Rogers, S.M., Simpson, S.J., 2014. Thanatosis. Curr. Biol. 24, R1031-R1033.

Romanczuk, P., Bar, M., Ebeling, W., Linder, B., Schimansky-Geier, L., 2012. Active Brownian particles from individuals to collective stochastic dynamics. Eur. Phys. J. - Spec. Top. 202, 1-162.

Röser, C., Jordan, N., Balfanz, S., Baumann, A., Walz, B., Baumann, O., Blenau, W., 2012. Molecular and pharmacological characterization of serotonin 5-HT2 $\alpha$ and 5-HT7 receptors in the salivary glands of the blowfly Calliphora vicina. PLoS ONE 7, e49459.

Rotte, C., Krach, C., Balfanz, S., Baumann, A., Walz, B., Blenau, W., 2009. Molecular characterization and localization of the first tyramine receptor of the American cockroach (Periplaneta americana). Neuroscience 162, 1120-1133.

Saudou, F., Boschert, U., Amlaiky, N., Plassat, J.L., Hen, R., 1992. A family of Drosophila serotonin receptors with distinct intracellular signalling properties and expression patterns. EMBO J 11.1, 7-17.

Saudou, F., Amlaiky, N., Plassat, J.L., Borelli, E., Hen, R., 1990. Cloning and characterization of a Drosophila tyramine receptor. EMBO J. 9, 3611-17.

Schaefer, G.W., 1969. Radar studies of locust, moth and butterfly migration in the Sahara. Proc. Roy. Entomol. Soc. London, C34, 33, 39-40.

Schaefer, G.W., 1976. Radar observations of insect flight, in: Rainey, R.C. (Ed.), Insect Flight Symp. Roy. Entomol. Soc., Blackwell, Oxford, U.K., Vol. 7, 157-197.

Schlenstedt, J., Balfanz, S., Baumann, A., Blenau, W., 2006. Am5-HT7: molecular and pharmacological characterization of the first serotonin receptor of the honeybee (Apis mellifera). J. Neurochem. 98, 1985-1998.

Shand, J. D., 2016. The effects of density-dependent polyphenism on circadian biology of the desert locust Schistocerca gregaria. Unpublished Ph.D Thesis, University of Leicester, U.K.

Shand, J. D., Matheson, T., Rosato, E., 2015. Circadian rhythmicity in behavioural and neuronal sensitivity in locusts. Proc. $35^{\text {th }}$ Göttingen Neurobiol. Conf. T14-1B. 
Simões, P.M., Niven, J.E., Ott, S.R., 2013. Phenotypic transformation affects associative learning in the desert locust. Curr. Biol. 23, 2407-2412.

Simões, P.M.V., Ott, S.R., Niven, J.E., 2011. Associative olfactory learning in the desert locust, Schistocerca gregaria. J. Exp. Biol. 214, 2495-503.

Simões, P.M.V., Ott, S.R., Niven, J.E., 2012. A long-latency aversive learning mechanism enables locusts to avoid odours associated with the consequences of ingesting toxic food. J. Exp. Biol. 215, $1711-1719$.

Simões, P.M.V., Ott, S.R., Niven, J.E., 2016. Environmental adaptation, phenotypic plasticity, and associative learning in insects: The Desert Locust as a case study. Integr. Comp. Biol. 56, 914924.

Simpson, S.J., 1981. An oscillation underlying feeding and a number of other behaviours in fifth instar Locusta migratoria nymphs. Physiol. Entomol. 6, 315-324.

Simpson, S.J., 1982. Patterns in feeding: a behavioural analysis using Locusta migratoria nymphs. Physiol. Entomol. 7, 325-336.

Simpson, S.J., Despland, E., Hägele, B.F., Dodgson, T., 2001. Gregarious behavior in desert locusts is evoked by touching their back legs. Proc. Natl. Acad. Sci. U.S.A. 98, 3895-3897.

Simpson, S.J., McCaffery, A.R., Hägele, B.F., 1999. A behavioural analysis of phase change in the desert locust. Biol. Rev. 74, 461-480.

Simpson, S.J., Sword, G.A., 2009. Phase polyphenism in locusts: Mechanisms, population consequences, adaptive significance and evolution, in: Whitman, D.W., Ananthakrishnan, T.N. (Eds.), Phenotypic Plasticity of Insects Mechanisms and Consequences. Science Publishers, Enfield (NH), pp. 140-190.

Sivanpillai, R., Latchininsky, A.V., 2008, Can late summer Landsat data be used for mapping Asian migratory locust, Locusta migratoria migratoria, oviposition sites in the Amudarya River delta, Uzbekistan? Entomol. Exp. Appl. 128, 346-353.

Sivanpillai, R., Latchininsky, A.V., 2007. Mapping locust habitats in Amudarya River Delta, Uzbekistan with multi-temporal MODIS imagery. Environ. Manage. 39, 876-886.

Sivanpillai, R., Latchininsky, A.V., Driese, K.L., Kambulin, V.E., 2006. Mapping locust habitats in River Ili Delta, Kazakhstan, using Landsat imagery. Agr. Ecosyst. Environ. 117, 128-134.

Smith, A.D., Riley, J.R., Gregory, R.D., 1993. A method for routine monitoring of the aerial migration of insects by using a vertical-looking radar. Phil. Trans. Roy. Soc. B 340, 393-404.

Smith, J., 2012. Application of Remote Sensing to Moroccan locust (Dociostaurus maroccanus Thunberg, 1815) Habitat in Southern Uzbekistan. Unpublished M.S. Thesis, University of Wyoming, Laramie, WY, U.S.A.

Song, H., 2005. Phylogenetic perspectives on the evolution of locust phase polyphenism. J. Orthoptera Res. 14, 235-245. 
Song, H., 2011. Density-dependent phase polyphenism in nonmodel locusts: A minireview. Psyche, Article ID 741769.

Song, H., Foquet, B., Mariño-Pérez, R. and Woller, D.A., in press. Phylogeny of locusts and grasshoppers reveals complex evolution of density-dependent phenotypic plasticity. Scientific Reports.

Song, H., Moulton, M.J., Hiatt, K.D., Whiting, M.F., 2013. Uncovering historical signature of mitochondrial DNA hidden in the nuclear genome: the biogeography of Schistocerca revisited. Cladistics 29, 643-662.

Song, H., Wenzel, J.W., 2008. Phylogeny of bird-grasshopper subfamily Cyrtacanthacridinae (Orthoptera: Acrididae) and the evolution of locust phase polyphenism. Cladistics 24, 515-542.

Spielman, S.J., Kumar, K., Wilke, C.O., 2015. Comprehensive, structurally-informed alignment and phylogeny of vertebrate biogenic amine receptors. PeerJ 3, e773.

Srivastava, D. P., Yu, E.J., Kennedy, K., Chatwin, H., Reale, V., Hamon, M., Smith, T., Evans, P.D., 2005. Rapid, nongenomic responses to ecdysteroids and catecholamines mediated by a novel Drosophila G-protein-coupled receptor. J. Neurosci. 25.26, 6145-6155.

Stamo, P.D., Kovalenkov V.G., Kuznetsova O.V., Nikitenko Y.V., 2013. Moroccan locust again in Stavropol. Plant Protection and Quarantine 2, 14-20 (in Russian).

Standage, D.S., Berens, A.J., Glastad, K.M., Severin, A.J., Brendel, V.P., Toth, A.L., 2016. Genome, transcriptome and methylome sequencing of a primitively eusocial wasp reveal a greatly reduced DNA methylation system in a social insect. Mol. Ecol. 25, 1769-1784.

Steedman, A. (Ed.), 1990. Locust handbook, third ed. Natural Resources Institute, Chatham.

Stettin, K., 2015. Mechanisms driving the acquisition and maintenance of gregarious behaviour in the desert locust (Schistocerca gregaria). Unpublished Ph.D Thesis, University of Cambridge, U.K.

Stettin, K., Ott, S.R., 2012. Phase change in the desert locust: A novel computerised behavioural assay to study the effects of anaesthesia on gregarisation. Front. Behav. Neurosci. Conf. Abstr: $10^{\text {th }}$ Int. Congr. Neuroethol. doi: 10.3389/conf. fnbeh.2012.27.00278.

Stige, L.C., Chan, K.S., Zhang, Z., Frank, D., Stenseth, N.C., 2007. Thousand-year-long Chinese time series reveals climate forcing of decadal locust dynamics. Proc. Natl. Acad. Sci. U.S.A. 104, $16188-16193$.

Stower, W.J., 1963. Photographic techniques for the analysis of locust "hopper" behaviour. Anim. Behav. 11, 199-205.

Sugahara, R., Tanaka, S., Jouraku, A., Shiotsuki, T., 2016. Functional characterization of the corazonin-encoding gene in phase polyphenism of the migratory locust, Locusta migratoria (Orthoptera: Acrididae). Appl. Entomol. Zool. 51, 225-232. 
Sugamori, K. S., Demchyshyn, L.L., McConkey, F., Forte, M.A., Niznik, H.B., 1995. A primordial dopamine D1-like adenylyl cyclase-linked receptor from Drosophila melanogaster displaying poor affinity for benzazepines. FEBS Lett. 362, 131-38.

Sumpter, D.J., 2010. Collective Animal Behaviour. Princeton University Press, Princeton.

Sun, W., Dong, H., Gao, Y.-B., Su, Q.-F., Qian, H.-T., Bai, H.-Y., Zhang, Z.-T., Cong, B., 2015. Genetic variation and geographic differentiation among populations of the nonmigratory agricultural pest Oedaleus infernalis (Orthoptera: Acridoidea). China. J. Insect Sci. 15, 150.

Sword, G.A., 1999. Density-dependent warning coloration. Nature 397, 217.

Sword, G.A., 2002. A role of phenotypic plasticity in the evolution of aposematism. Proc. Roy. Soc. B Biol. Sci. 269, 1639-1644.

Sword, G.A., 2003. To be or not to be a locust? A comparative analysis of behavioral phase change in nymphs of Schistocerca americana and S. gregaria. J. Insect Physiol. 49, 709-717.

Sword, G.A., Lecoq, M., Simpson, S.J., 2010. Phase polyphenism and preventative locust management. J. Insect Physiol. 56, 949-957.

Sword, G.A., Lorch, P.D., Gwynne, D.T., 2005. Insect behaviour: Migratory bands give crickets protection. Nature 433, 703.

Sword, G.A., Simpson, S.J., 2008. Locusts. Curr. Biol. 18, 364-366.

Sword, G.A., Simpson, S.J., El Hadi, O.M., Wilps, H., 2000. Density-dependent aposematism in the desert locust. Proc. Roy. Soc. B Biol. Sci. 267, 63-68.

Symmons, P.M., 1992. Strategies to combat desert locust. Crop Prot. 11, 206-212.

Szyf, M., McGowan, P., Meaney, M.J., 2008. The social environment and the epigenome. Environ. Mol. Mutagenesis 49, 46-60.

Tahir, N., Brooker, G., 2015. Toward the development of millimeter wave harmonic sensors for tracking small insects. IEEE Sens. J. 15, 5669-5676.

Tanaka, S., Nishide, Y., 2013. Behavioral phase shift in nymphs of the desert locust, Schistocerca gregaria: Special attention to attraction/avoidance behaviors and the role of serotonin. J. Insect Physiol. 59, 101-112.

Thamm, M., Balfanz, S., Scheiner, R., Blenau, W., 2010. Characterization of the 5-HT1A receptor of the honeybee (Apis mellifera) and involvement of serotonin in phototactic behavior. Cell. Mol. Life Sci. 67, 2467-2479.

Tian, H.D., Ji, R., Xie, B.Y., Li, X.H., Li, D.M., 2008. Using multi-temporal Landsat ETM+ data to monitor the plague of oriental migratory locust. Int. J. Remote Sens. 29, 1685-1692.

Tian, H., Stige, L.C., Cazelles, B., Kausrud, K.L., Svarverud, R., Stenseth, N.C., Zhang, Z., 2011. Reconstruction of a 1,910-y-long locust series reveals consistent associations with climate fluctuations in China. Proc. Natl. Acad. Sci. U.S.A. 108, 14521-14526. 
Tierney, A. J., 2001, Structure and function of invertebrate 5-HT receptors: a review. Comp Biochem. Physiol. A Mol. Integr. Physiol. 128, 791-804.

Tobback, J., Boerjan, B., Vandersmissen, H.P. and Huybrechts, R., 2011. The circadian clock genes affect reproductive capacity in the desert locust Schistocerca gregaria. Insect Biochem. Mol. Biol. 41, 313-321.

Tobback, J., Boerjan, B., Vandersmissen, H.P. and Huybrechts, R., 2012. Male reproduction is affected by RNA interference of period and timeless in the desert locust Schistocerca gregaria. Insect Biochem. Mol. Biol. 42, 109-115.

Tokuda, M., Tanaka, S., Zhu, D.H., 2010. Multiple origins of Locusta migratoria (Orthoptera: Acrididae) in the Japanese Archipelago and the presence of two major clades in the world: evidence from a molecular approach. Biol. J. Linn. Soc. 99, 570-581.

Tomioka, K., Matsumoto, A., 2010. A comparative view of insect circadian clock systems. Cell. Mol. Life Sci. 67, 1397-1406.

Tomioka, K., Wakatsuki, T., Shimono, K., Chiba, Y., 1991. Circadian control of hatching in the cricket, Gryllus bimaculatus. J. Insect Physiol. 37, 365-371.

Tong X.-W., Chen B., Huang L-H., Feng Q.-L., Kang, L., 2015. Proteomic analysis reveals that COP9 signalosome complex subunit 7A (CSN7A) is essential for the phase transition of migratory locust. Sci. Rep. 5, 12542.

Torney, C.J., Berdahl, A., Couzin, I.D., 2011. Signalling and the Evolution of Cooperative Foraging in Dynamic Environments. PLoS Comput. Biol. 7, e1002194.

Tratalos, J.A., Cheke, R.A., 2006. Can NDVI GAC imagery be used to monitor desert locust breeding areas? J. Arid Environ. 64, 342-356.

Tronin, A.A., Gornyy, V.I., Kiselev, A.V., Kritsuk, S.G., Latypov, I.S., 2014. Forecasting of locust mass breeding by using satellite data. Modern Problems of Earth Remote Sensing from Space 11, 137-150.

Troppmann, B., Balfanz, S., Krach, C., Baumann, A., Blenau, W., 2014. Characterization of an invertebrate-type dopamine receptor of the American cockroach, Periplaneta americana. Int. J Mol. Sci. 15, 629-653.

Troppmann, B., Balfanz, S., Baumann, A., Blenau, W., 2010. Inverse agonist and neutral antagonist actions of synthetic compounds at an insect 5-HT1 receptor. Brit. J. Pharmacol. 159, 1450-1462.

Trujillo G.P., 1975. El problema de la langosta Schistocerca paranensis Burm. Sociedad de Geografía y Estadística de Baja california, Tijuana, México.

Tucker, C.J., Hielkema, J.U., Roffey, J., 1985. The potential of satellite remote sensing of ecological conditions for survey and forecasting desert-locust activity. Int. J. Remote Sens. 6, 127138. 
Tyrer, N.M., Pozza, M.F., Humbel, U., Peters, B.H., Bacon, J.P., 1988. The tritocerebral commissure dwarf (TCD) - a major GABA-immunoreactive descending interneurone in the locust. J. Comp. Physiol. A 164, 141-150.

Uvarov B., 1966. Grasshoppers and Locusts. Vol. 1, Cambridge Uni. Press, Cambridge.

Uvarov B., 1977. Grasshoppers and Locusts. Vol. 2, Centre for Overseas Pest Research, London.

Van der Werf, W., Woldewahid, G., Van Huis, A., Butrous, M., Kora K.Y., 2005. Plant communities can predict the distribution of solitarious desert locust Schistocerca gregaria. J. App. Ecol. 42, 989-997.

Vanden Broeck, J., Vulsteke, V., Huybrechts, R., De Loof, A., 1995. Characterization of a cloned locust tyramine receptor cDNA by functional expression in permanently transformed Drosophila S2 cells. J. Neurochem. 64, 2387-2395.

Veran, S., Simpson, S.J., Sword, G.A., Deveson, E., Piry, S., Hines, J.E., Berthier, K., 2015 Modeling spatiotemporal dynamics of outbreaking species: influence of environment and migration in a locust. Ecology, 96, 737-748.

Vergoz, V., McQuillan, H.J., Geddes, L.H., Pullar, K., Nicholson, B.J., Paulin, M.G., Mercer, A.R., 2009. Peripheral modulation of worker bee responses to queen mandibular pheromone. Proc. Natl. Acad. Sci. U.S.A. 106, 20930-20935.

Verlinden, H., Badisco, L., Marchal, E., Van Wielendaele, P., Vanden Broeck J., 2009. Endocrinology of reproduction and phase transition in locusts. Gen. Comp. Endocrin. 162, 79-92.

Verlinden, H., Vleugels, R., Marchal, E., Badisco, L., Pflüger, H., Blenau, W., Vanden Broeck., 2010a. The role of octopamine in locusts and other arthropods. J. Insect Physiol. 56, 854-867.

Verlinden, H., Vleugels, R., Marchal, E., Badisco, L., Pflüger, H., Blenau, W., Vanden Broeck., $2010 \mathrm{~b}$. The cloning, phylogenetic relationship and distribution pattern of two new putative GPCRtype octopamine receptors in the desert locust (Schistocerca gregaria). J. Insect Physiol. 56, 868875.

Verlinden, H., Vleugels, R., Verdonck, R., Urlacher, E., Vanden Broeck, J., Mercer, A., 2015. Pharmacological and signalling properties of a D2-like dopamine receptor (Dop3) in Tribolium castaneum. Insect Biochem. Mol. Biol. 56, 9-20.

Verlinden, H., Vleugels, R., Zels, S., Dillen, S., Lenaerts, C., Crabbé, K., Spit, J., Vanden Broeck, J., 2014. Receptors for neuronal of endocrine signalling molecules as potential targets for the control of insect pests. Adv. Insect Physiol. 46, 167-303.

Vicsek, T., Czirók, A., Ben-Jacob, E., Cohen, I., Shochet, O., 1995. Novel type of phase transition in a system of self-driven particles. Phys. Rev. Lett. 75, 1226-1229.

Vinatier, F., Tixier, P., Duyck, P-F, Lescuarret, F., 2011. Factors and mechanisms explaining spatial heterogeneity: a review of methods for insect populations. Methods Ecol. Evol. 2, 11-22. 
Vleugels, R., Lenaerts, C., Baumann, A., Vanden Broeck, J., Verinden, H., 2013. Pharmacological characterization of a 5-HT1-type serotonin receptor in the red flour beetle, Tribolium castaneum. PLoS ONE 8, e65052.

Vleugels, R., Lenaerts, C., Vanden Broecks., Verlinden, H., 2014. Signalling properties and pharmacology of a 5-HT7-type serotonin receptor from Tribolium castaneum. Insect Mol. Biol. $23,230-43$.

Vleugels, R., H. Verlinden., J. Vanden Broeck., 2015. Serotonin, serotonin receptors and their actions in insects. Neurotransmitter 2, e314.

Voss, F., Dreiser, U., 1994. Mapping of Desert locust and other migratory pests habitats using remote sensing techniques, in: Krall S., Wilps H. (Eds.), New trends in locust control. GTZ Schriftenreiche, Eschborn control, vol. 245, pp. 23-29.

Voss, F., Dreiser, U., 1997. Mapping of desert locust habitats using remote sensing techniques, in: Krall, S., Peveling. R., Ba Diallo, D., (Eds.), New strategies in locust control. Birkhauser Verlag, Basel, pp. 37-45.

Waldner, F., Babah Ebbe, M.A., Cressman, K., 2015. Geo-information operational monitoring of the desert locust habitat with earth Observation: an assessment. ISPRS Int. J. Geo-Inform. 4, 23792400 .

Waloff, Z., Pedgley, D.E., 1986. Comparative biogeography and biology of the South American locust, Schistocerca cancellata (Serville), and the South African desert locust, S. gregaria flaviventris (Burmeister) (Orthoptera: Acrididae): a review. Bull. Entomol. Res. 76, 1-20.

Wang, X., Fang, X., Yang, P., Jiang, X., Jiang, F., Zhao, D., Li, B, Cui, F., Wei, J., Ma, C., Wang, Y., He, J., Luo, Y., Wang, Z., Guo, X., Guo, W., Wang, X., Zhang, Y., Yang, M., Hao, S., Chen, B., Ma, Z., Yu, D., Xiong, Z., Zhu, Y., Fan, D., Han, L., Wang, B., Chen, Y., Wang, J., Yang, L., Zhao, W., Feng, Y., Chen, G., Lian, J., Li, Q., Huang, Z., Yao, X., Lv, N., Zhang, G., Li, Y., Wang, J., Wang, J., Zhu, B., Kang, L., 2014. The locust genome provides insight into swarm formation and long-distance flight. Nature Comm. 5, 2957.

Wang, X., Kang, L., 2014. Molecular mechanisms of phase change in locusts. Annu. Rev. Entomol. 59, 225-244

Wei, Y., Chen, S., Yang, P., Ma, Z., Kang, L., 2009. Characterization and comparative profiling of the small RNA transcriptomes in two phases of locust. Genome Biol. 10, R6.

Weiss, J.E.R., 2016. Do locusts seek greener pastures? An evaluation of MODIS vegetation indices to predict presence, abundance and impact of the Australian plague locust in south-eastern Australia. Unpublished Ph.D Thesis, University of Melbourne, Australia.

Westerman, M., Barton, N.H., Hewitt GM., 1987. Differences in DNA content between two chromosomal races of the grasshopper Podisma pedestris. Heredity 58, 221-228

Witz, P., Amlaiky, N., Plassat, J.L., Maroteaux, L., Borelli, E., Hen, R., 1990. Cloning and characterization of a Drosophila serotonin receptor that activates adenylate cyclase. Proc. Natl. Acad. Sci. U.S.A. 87, 8940-8944. 
Woldewahid, G., Van der Werf, W., Sykora, K., Abate, T., Mostafa, B., Van Huis, A., 2007. Description of plant communities on the Red Sea coastal plain of Sudan. J. Arid Environ. 68, 113131.

Woldewahid, G., Van Der Werf, W., Van Huis, A., Steins, A., 2004. Spatial distribution of populations solitarious adult desert locust (Schistocerca gregaria Forsk.) on coastal plain of Sudan. Agric. Forest Entomol. 6, 181-191.

Wu, S.F., Huang, J., Ye, G.Y., 2013. Molecular cloning and pharmacological characterisation of a tyramine receptor from the rice stem borer, Chilo suppressalis (Walker). Pest. Manag. Sci. 69, 126-134.

Wu, S.F., Yao, Y., Huang, J., Ye, G., 2012a. Characterization of a beta-adrenergic-like octopamine receptor from the rice stem borer (Chilo suppressalis). J. Exp. Biol. 215, 2646-2652.

Wu, S.F., Xu, G., Xia, R.Y., Huang, J., Ye, G.Y., 2014. Two splicing variants of a novel family of octopamine receptors with different signaling properties. J. Neurochem. 129, 37-47.

Wu, S.F., Xu, G., Ye, G.Y., 2015. Characterization of a tyramine receptor type 2 from hemocytes of rice stem borer, Chilo suppressalis. J. Insect Physiol. 75, 39-46.

Wu, T., Hao, S., Sun, O.J., Kang, L., 2012b. Specificity responses of grasshoppers in temperate grasslands to diel asymmetric warming. PLoS ONE 7, e41764.

Wynant, N., Verlinden, H., Breugelmans, B., Simonet, G., Vanden Broeck, J., 2012. Tissuedependence and sensitivity of the systemic RNA interference response in the desert locust, Schistocerca gregaria. Insect Biochem. Mol. Biol. 42, 911-917.

Wynant, N., Santos, D., Subramanyam, S.H., Verlinden, H., Vanden Broeck, J., 2015. Drosha, Dicer-1 and Argonaute-1 in the desert locust: Phylogenetic analyses, transcript profiling and regulation during phase transition and feeding. J. Insect Physiol. 75, 20-29.

Xian-Lei, Q., Xian-Hui, W., Hong-Fu, X., Kang, L., 2007. Influence of soil moisture on egg cold hardiness in the migratory locust Locusta migratoria (Orthoptera: Acrididae). Physiol. Entomol. $32,219-224$.

Xu, L., Li, L., Yang, P., Ma, Z., 2017. Calmodulin as a downstream gene of octopamine-OAR a1 signalling mediates olfactory attraction in gregarious locusts. Insect Mol. Biol. 26, 1-12.

Yan, H., Bonasio, R., Simola, D.F. Liebig, J., Berger, S.L., Reinberg, D., 2015. DNA methylation in social insects: how epigenetics can control behavior and longevity. Annu. Rev. Entomol. 60, 435-452.

Yates, C.A., Erban, R., Escudero, C., Couzin, I.D., Buhl, J., Kevrekidis, I.G., Sumpter, D.J., 2009. Inherent noise can facilitate coherence in collective swarm motion. Proc. Natl. Acad. Sci. U.S.A. $106,5464-5469$.

Young, O.R., 2002. The Institutional Dimensions of Environmental Change: Fit, Interplay and Scale. MIT Press, Cambridge, MA. 
Yu, G., Shen, H., Liu, J., 2009. Impacts of climate change on historical locust outbreaks in China. J. Geogr. Res. 114, D18104.

Zha, Y., Gao, J., Ni, S., Shen, N., 2005. Temporal filtering of successive MODIS data in monitoring a locust outbreak. Int. J. Remote Sens. 26, 5665-5674.

Zhang, Z., Cazelles, B., Tian, H., Stige, L.C., Bräuning, A., Stenseth, N.C., 2009. Periodic temperature-associated drought/flood drives locust plagues in China. Proc. Roy. Soc. B Biol. Sci. 276, 823-831.

Zhang, Z., Peng, Z.-Y., Yi, K., Cheng, Y., Xia, Y., 2012. Identification of representative genes of the central nervous system of the locust, Locusta migratoria manilensis by deep sequencing. J. Insect Sci. 12, 1-15.

Figure 1. Diversity of nymphal colour patterns within the genus Schistocerca. (a) S. lineata, which specialises on Ptelea trifoliata, reared at high density. This species shows density-dependent aposematic colouration. (b) S. nitens caribbeana reared in isolation. This species does not show density-dependent colour plasticity, but exhibits a green-brown polymorphism irrespective of rearing density. (c) S. damnifica reared at high density. Isolated nymphs are typically green. (d) $S$. ceratiola reared in isolation. This species does not change colour when crowded. (e) S. americana reared in isolation (left) and under crowded conditions (right). (f) S. piceifrons reared at high density. (g) S. serialis cubense reared at high density. This species shows a very reduced level of colour plasticity. (h) S. nitens nitens reared at high density. Like S. nitens caribbeana, this species does not show density-dependent colour plasticity but exhibits a green-brown polymorphism that does not appear to be linked to rearing density.

Figure 2. Human - locust interactions. Locust (brown lines) population growth can lead to phase change and large-scale migration, which impacts livelihoods both locally and in distant regions. Regions are connected through markets, policy, and government agencies (black lines). These forces affect farmer decision making and land use outcomes, which in turn affect soil and plant parameters (green lines). In addition to weather (blue lines), these parameters influence locust population growth and the probability that locusts will undergo a series of neurophysiological, genetic, morphological, and behavioural changes that lead to collective movement and large-scale migration. Monitoring and control (grey lines) typically happens at the level of state or national governments, or international cooperations. Remote sensing (orange lines) has become an increasingly important tool for research and management. Solid lines indicate known connections; dotted lines indicate suspected but unproven connections. 


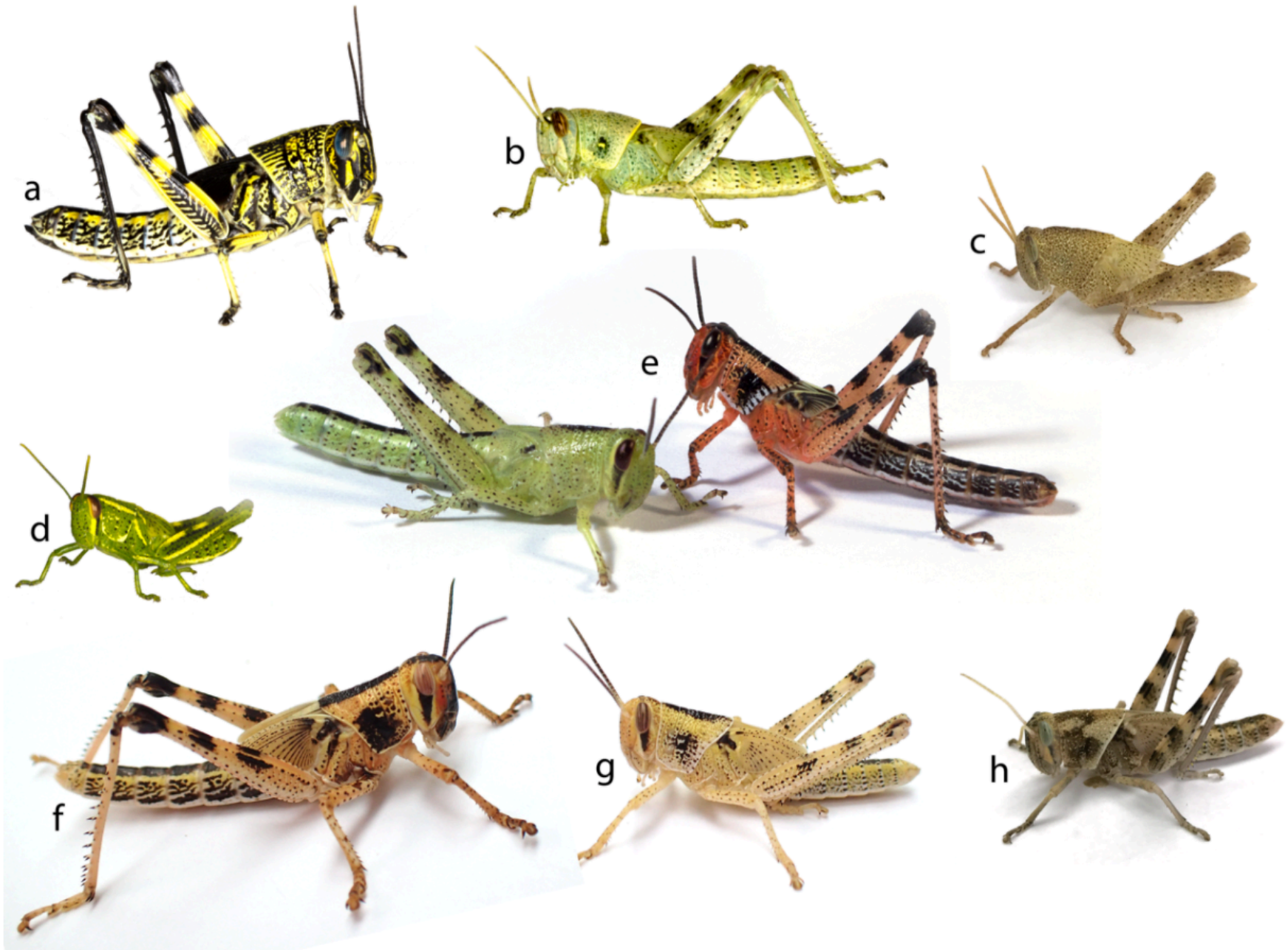




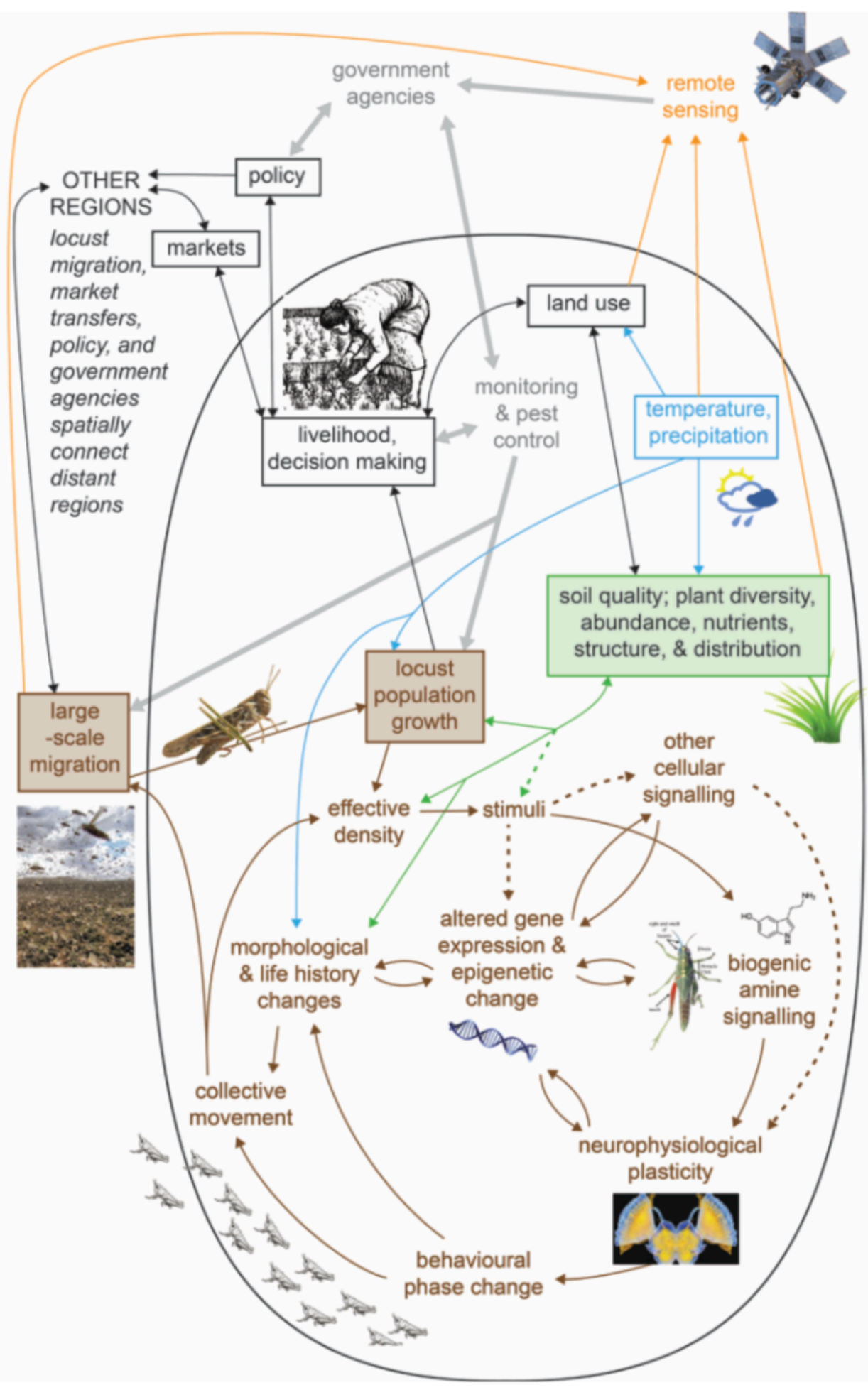




\begin{tabular}{|c|c|c|}
\hline Scientific name (Locusts) & Common name & Geographic range \\
\hline Anacridium melanorhodon (Walker, 1870) & Sahelian Tree Locust & North and Central Africa, Middle East \\
\hline Aiolopus simulatrix (Walker, 1870) & Sudan Plague Locust & North and Central Africa \\
\hline Austracris guttulosa (Walker, 1870) & Spur-Throated Locust & Australia \\
\hline Calliptamus italicus (Linnaeus, 1758) & Italian Locust & Europe and Central Asia \\
\hline Ceracris kiangsu Tsai, 1929 & Yellow-Spined Bamboo Locust & East Asia \\
\hline Chortoicetes terminifera $($ Walker, 1870$)$ & Australian Plague Locust & Australia \\
\hline Dociostaurus maroccanus (Thunberg, 1815) & Moroccan Locust & North Africa, Europe, Caucasus \\
\hline Gastrimargus musicus (Fabricius, 1775) & Yellow-Winged Locust & Australia \\
\hline Gomphocerus sibiricus (Linnaeus, 1767) & Siberian Locust & Europe, Caucasus, North Asia \\
\hline Locusta migratoria migratoria (Linnaeus, 1758 ) & Asian Migratory Locust & West and Central Asia, Eastern Europe \\
\hline Locusta migratoria migratorioides (Reiche and Fairmaire, 18 & African Migratory Locust & Mainland Africa and Atlantic Islands, Madagascar, South and East Asia, Australasia, Tibetan plateau \\
\hline Locustana pardalina (Walker, 1870$)$ & Brown Locust & Southern Africa \\
\hline Nomadacris septemfasciata Audinet-Serville, 1883 & Red Locust & Sub-Saharan and Southern Africa \\
\hline Oedaleus asiaticus Bey-Bienko, 1941 & Mongolian Locust & Northern Asia \\
\hline Oedaleus senegalensis (Krauss, 1877) & Senegalese Locust & North Africa, Middle East \\
\hline Patanga succincta (Johansson, 1763) & Bombay Locust & India, South-East Asia \\
\hline Schistocerca cancellata (Serville, 1838) & South American Locust & South America \\
\hline Schistocerca gregaria flaviventris (Burmeister, 1838) & Desert Locust (southern race) & Southern Africa (N.B., typically non-swarming) \\
\hline Schistocerca gregaria gregaria (Forskål, 1775) & Desert Locust (northern race) & Southern Europe, North Africa, Middle East, Indian sub-continent \\
\hline Schistocerca interrita Scudder, 1899 & Peruvian Locust & Peru \\
\hline Schistocerca piceifrons peruviana Lynch Arribalzaga, 1903 & Central American Locust & Peru, Ecuador, Colombia, Venezuela, Panama, Trinidad and Tobago \\
\hline Schistocerca piceifrons piceifrons (Walker, 1870) & Central American Locust & Mexico, northern Costa Rica \\
\hline Scientific name (Non-locusts) & Common name & Geographic range \\
\hline Aiolopus thalassinus (Fabricius, 1781) & Slender Green-Winged Grasshopper & Africa, Europe, Asia \\
\hline Anabrus simplex Haldeman, 1852 & Mormon Cricket & North America \\
\hline Calliptamus barbarus (Costa, 1836) & Eurasian Pincer Grasshopper & North Africa, Europe, West and Central Asia \\
\hline Calliptamus wattenwylianus Pantel, 1896 & Western Pincer Grasshopper & North Africa, Europe \\
\hline Camnula pellucida (Scudder, 1862) & Clear-Winged Grasshopper & North America \\
\hline Chorthippus parallelus (Zetterstedt, 1821) & Meadow Grasshopper & Great Britain, Europe, Caucasus, West and Central Asia \\
\hline Coscineuta virens (Thunberg, 1815) & Moruga Grasshopper & Trinidad and Tobago \\
\hline Melanoplus differentialis (Thomas, 1865) & Differential Grasshopper & North America \\
\hline Melanoplus sanguinipes (Fabricius, 1798) & Migratory Grasshopper & North America \\
\hline Oedaleus infernalis Saussure, 1884 & & Caucasus, Central and East Asia, Japan \\
\hline Rhammatocerus schistocercoides (Rehn, 1906) & Mato Grosso Grasshopper & Brazil \\
\hline Schistocerca americana (Drury, 1770) & American Bird Grasshopper & North America \\
\hline Schistocerca ceratiola (Hubbell and Walker, 1928) & Florida Rosemary Grasshopper & Florida \\
\hline Schistocerca damnifica (Saussure, 1861$)$ & Mischievous Grasshopper & North America \\
\hline Schistocerca lineata Scudder, 1889 & Spotted Bird Grasshopper & North America \\
\hline Schistocerca nitens caribbeana Dirsh, 1974 & & Caribbean \\
\hline Schistocerca nitens nitens (Thunberg, 1815) & Gray Bird Grasshopper & North America, Mexico \\
\hline Schistocerca serialis cubense (Saussure, 1861) & & Florida Keys, Caribbean, Cuba, Jamaica \\
\hline
\end{tabular}

